EUROPEAN ORGANIZATION FOR NUCLEAR RESEARCH

CERN-EP/2002-008

21 January 2002

\title{
CONSTRUCTION, TEST AND COMMISSIONING OF THE TRIPLE-GEM TRACKING DETECTOR FOR COMPASS
}

\author{
C. Altunbas ${ }^{1}$, M. Capéans ${ }^{2,3}$, K. Dehmelt ${ }^{3,6}$, J. Ehlers ${ }^{3,6}$, J. Friedrich ${ }^{5}$, \\ I. Konorov ${ }^{5}$, A. Gandi ${ }^{2}$, S. Kappler ${ }^{2,4}$, B. Ketzer ${ }^{2}$, R. De Oliveira ${ }^{2}$, S. Paul ${ }^{5}$, \\ A. Placci ${ }^{2}$, L. Ropelewski ${ }^{2}$, F. Sauli ${ }^{2 *}$, F. Simon ${ }^{5}$, M. van Stenis ${ }^{2}$ \\ ${ }^{1}$ SUNY Buffalo, Department of Physics, Amherst, NY, USA \\ ${ }^{2}$ CERN, Geneva, Switzerland \\ ${ }^{3}$ Physikalisches Institut, Universität Heidelberg, Germany \\ ${ }^{4}$ Institut für Experimentelle Kernphysik, Universität Karlsruhe, Germany \\ ${ }^{5}$ Physik-Department, Technische Universität München, Garching, Germany \\ ${ }^{6}$ Physikalisches Institut, Universität Mainz, Germany
}

\begin{abstract}
The Small Area Tracking system of the COMPASS experiment at CERN includes a set of 20 large area, fast position-sensitive Gas Electron Multiplier (GEM) detectors, designed to reliably operate in the harsh radiation environment of the experiment. We describe in detail the design, choice of materials, assembly procedures and quality controls used to manufacture the devices. The test procedure in the laboratory, the performance in test beams and in the initial commissioning phase in the experiment are presented and discussed.
\end{abstract}

Keywords: Gas Electron Multiplier; GEM; COMPASS; fast gas detector; high rate tracking.

PAC98: 29.40.Cs; 51.50.+v

Submitted to Nuclear Instruments and Methods in Physics Research (Sect. A)

*Corresponding author: fabio.sauli@cern.ch 



\section{INTRODUCTION}

The COMPASS experiment (COmmon Muon and Proton Apparatus for Structure and Spectroscopy) is being commissioned from the summer '01 in the EH-N2 hall at the CERN Super Proton Synchrotron (SPS) [1]. Its main purpose is to investigate hadron structure and spectroscopy using high intensity polarized muon and hadron beams interacting with a target. One of the main goals of the experiment is to measure the gluon contribution to the nucleon spin, both from the asymmetry in cross section for open charm production in deep inelastic scattering of polarized muons on polarized nucleons, and from asymmetries in the production of high- $\mathrm{p}_{\mathrm{T}}$ hadron jets. Polarized ${ }^{6} \mathrm{LiD}$ for neutron spin structure, and $\mathrm{NH}_{3}$ for proton spin structure act as targets for the $100-190 \mathrm{GeV} / \mathrm{c}$ polarized muon beam.

The hadronic program consists of various topics. It includes Primakoff scattering, which is Compton scattering in inverse kinematics, to study electric and magnetic polarizabilities of the beam hadrons and search of exotic non- $q \bar{q}$ mesons-like $f_{0}(1500)$ (glueballs). Studies of the influence of strong interaction and spectator effects on the weak decay of hadrons is performed analyzing the charm hadron decays. For these tasks, a liquid hydrogen target for the central production of mesons and gluon-rich states is used, while a thin copper plate acts as a charm-producing target. COMPASS is a natural continuation of previous experiments addressing similar physics goals [2-4].

All physical observations require very high beam rates to obtain a large integrated luminosity, with intensities of up to $\sim 2.10^{8}\left(\sim 10^{8}\right)$ particles per $5 \mathrm{~s}$ spill in the muon (hadron) beam. This requires the use of detectors with very high rate and multi-track resolution capability, combined with fast, dead-time free readout electronics and efficient trigger selection. Moreover, in order to reduce background-producing secondary interactions, all devices, and particularly those close to the beam axis, have to be built with minimum mass.

As shown in Fig. 1, COMPASS is a two-stage magnetic spectrometer; both sections have outstanding facilities for particle tracking, identification and calorimetry. The first section, downstream from the target and including a momentum analyzing magnet (SM1 in the figure), covers an angular range up to $220 \mathrm{mrad}$ in the vertical and $250 \mathrm{mrad}$ in the horizontal direction. The 
second, after the second magnet (SM2), detects tracks below 30 mrad. Tracking between target and first magnet is implemented by a telescope of double-sided silicon micro-strips and scintillating fibers in the area closer to the beam, and four Micromegas gas detector planes with larger acceptance [5]. Downstream from the first magnet, the small angle tracker of the experiment is equipped with 20 chambers based on the Gas Electron Multiplier (GEM) technology [6]. Several stations equipped with layers of large-area drift chambers, multiwire chambers and straw tubes, having lower rate capability, are used to cover the larger angles; the GEM chambers are mechanically mounted on these devices. The detectors, with an active area of $31 \times 31 \mathrm{~cm}^{2}$ each, have two-dimensional Cartesian projective strip readout and are mounted in pairs, with one chamber rotated by $45^{\circ}$ in respect to the other, on ten stations along the spectrometer. As a result of extensive research, and to ensure reliable operation in presence of a large background of heavily ionizing tracks, we have adopted the triple-GEM configuration, which permits to achieve an almost discharge-free operation up to very high gains [7-10]. In order not to be flooded by the primary non-interacting beam, a central region $50 \mathrm{~mm}$ in diameter in each chamber can be disabled remotely. Fig. 2 shows the linear charged particle rate distributions, computed with a monte-carlo simulation, at a position $15 \mathrm{~m}$ from the target (GEM station 4) with the central beam killer activated, projected on the horizontal (x) and vertical (y) directions. The corresponding occupancies per strip for $50 \mathrm{~ns}$ gating are obtained taking into account the strip pitch $(400 \mu \mathrm{m})$ and the average cluster size. Rates have been estimated for a non-interacting highintensity muon beam ${ }^{1}$.

\section{CONSTRUCTION OF THE GEM DETECTORS}

\subsection{Overview}

To satisfy the low material budget requirement of the experiment, all components used for the chamber construction and the readout electronics have been selected for minimum mass, particularly in the beam region. Each detector is fabricated piling up and gluing together a set of thin frames holding the various electrodes, with mechanical stiffness and gas tightness provided by two light honeycomb outer supporting plates. To further reduce

\footnotetext{
${ }^{1}$ Computed by A. Bressan, INFN Trieste and CERN.
} 
the material, the plates have openings in the central region. The schematic of the COMPASS Triple-GEM assembly is shown in Fig. 3, with a detailed cross section given in Fig. 4. Starting from the top, one can recognize the upper honeycomb plate, the drift electrode, three GEM foils and the read-out board pasted over the lower honeycomb. The drift or sensitive volume is $3 \mathrm{~mm}$ thick, all others are $2 \mathrm{~mm}$ thick. On application of suitable voltages, electrons released by radiation in the topmost gas layer drift and multiply through the cascade of GEMs; the final patterned electrode collects the amplified charge on two sets of perpendicular strips, and permits two-dimensional localization. The central region, $50 \mathrm{~mm}$ in diameter, corresponding to the primary beam and near halo, can be remotely enabled acting on the potential of the last GEM foil.

The picture in Fig. 5 shows a fully assembled chamber, with the high voltage distribution and the electronics readout boards for the two coordinates. In actual operation, a thin aluminum-coated polymer foil is laid over the whole area for protection and screening. Table 1 specifies the components used in the assembly, and Table 2 provides the detailed material budget; choices reflect a compromise between sufficient rigidity and low mass, as well as the use of non-outgassing materials to minimize undesirable aging effects induced by the long-term exposure of the detectors to high particle rates [11]. A complete chamber amounts to about $0.7 \%$ of a radiation length in the active area, reduced to $\sim 0.5 \%$ in the central beam region.

\subsection{Honeycomb plates}

The main support and rigidity of the chamber is given by two honeycomb plates, $33 \times 33$ and $55 \times 50 \mathrm{~cm}^{2}$ in size respectively, made as a sandwich of thin fiberglass foils with a $3 \mathrm{~mm}$ thick cellular spacer (see Table 1). The plates are custom-made and cut to size in the CERN-EP-TA1 workshop; both have a hole cut-out in the center, $50 \mathrm{~mm}$ in diameter for the larger plate and $35 \mathrm{~mm}$ on the drift side ${ }^{2}$. Inserts with precision-drilled holes in the larger plate are used for fixing various ancillary components and for mounting the chambers on their final supports. This plate also houses the gas outlet, made with a fiberglass insert connected to the inner volume through holes on the corner of the readout foil. For screening , a $10 \mu \mathrm{m}$ aluminum foil is glued on the outside of the larger plate.

\footnotetext{
${ }^{2}$ To avoid deformation of the drift electrode during assembly, done on a vacuum table.
} 


\subsection{GEM electrodes}

The GEM electrodes consist of thin metal-coated polymer foils, pierced by a large number of holes. After extensive investigations we have adopted 50 $\mu \mathrm{m}$ kapton foils coated with $5 \mu \mathrm{m}$ copper; holes are arranged in a triangular pattern, and are $\sim 70 \mu \mathrm{m}$ in diameter at $140 \mu \mathrm{m}$ distance center to center [12]. Although other choices may be more performing or desirable, as for example smaller hole diameter and pitch or a thinner metal layer, they would affect the production yield and could not be adopted for the present production. Fig. 6 shows an electron microscope picture of the hole pattern, and Fig. 7 a detailed cross section revealing the slight double-conical profile of the holes, a consequence of the chemical etching process ${ }^{3}$.

The GEM manufacturing technology, developed by the CERN-ESTDEM workshop in strict collaboration with the users, has been outlined in earlier works $[6,12]$. The process begins with the production of two identical masks, whose pattern is transferred to the photo-resist coated foils, using conventional printed circuit technology, by exposure to UV light. For such large sizes, a crucial parameter is the precise alignment of the two masks. Indeed, since the patterned copper layer is used as self-mask during the chemical process of etching through the polymer, any misalignment results in slanted holes, yielding lower gain and prone to charge up. This is a particularly difficult requirement, due to the use of plastic masks that can deform under thermal stress. The shape of the through-holes, obtained by immersion of the patterned foil in a solvent, evolves depending on the etching time, from double-conical though cylindrical to inward going. Although a cylindrical shape would be the more desirable, we have found that, probably due to non-uniformities in the material, this may result in local defects with complete removal of the polymer and creation of fragile metal "bridges". To obtain a reasonable production yield, the minimum hole diameter in the center of the kapton foil has been specified to be $60 \mu \mathrm{m}$. Residual charging-up effects due to this choice will be discussed in section 4. Figs. 8 and 9 show distributions of the holes' diameter, measured in 6 points scattered over the area at the copper level and at the center of the kapton on ten production GEM foils. A tolerance of $\pm 2.5 \mu \mathrm{m}$ around the average value is requested for acceptance.

\footnotetext{
${ }^{3}$ Electron microscope pictures made by G. Jesse, CERN-EST-SM.
} 
To reduce both energy and propagation probability of discharges, and as a result of previous studies [7], we have subdivided one side of each GEM foil in 12 sectors, separately connected externally to the voltage supply through high value resistors. The separation between sectors, $200 \mu \mathrm{m}$, is sufficiently narrow to induce only a small perturbation in the uniformity of response of the detector ${ }^{4}$. An additional segmentation of the center, in the shape of a disk $50 \mathrm{~mm}$ in diameter, is independently powered and serves as beam killer during normal operation, but can be activated during low intensity runs for calibration and beam alignments. The other side of the multiplier is not segmented; under control of the resistive potential distribution (see 3.1) its voltage remains almost constant in case of a discharge, thus preventing propagation to other electrodes [7]. Fig. 10 shows the segmented side of a GEM foil, and Fig. 11 is a magnified view of the separation between segments. A close-up of the region corresponding to the electrical contact to the beam killer is shown in Fig. 12; to provide the voltage, a thin line runs in the middle of a larger gap ( $400 \mu \mathrm{m}$ wide) separating on one side the central sectors. To ensure uniform distribution of voltage, and to prevent diffusion of the glue into the holes during manufacturing, a $3 \mathrm{~mm}$ wide full metal border is left all around the active area of the multiplier; copper strips on kapton bring the various contacts outside the framed foil. To avoid instabilities, observed in early prototypes, care is taken to avoid the presence of partly metallized holes; all incomplete holes on the edge of the pattern are removed on the design of the mask.

\subsection{Drift electrode, support frames and spacers}

The drift electrode, made of a single-sided metal-coated kapton foil 50 $\mu \mathrm{m}$ thick, is directly glued on the small honeycomb plate, with the $5 \mu \mathrm{m}$ copper layer patterned to cover the active part of the detector only in order to prevent edge problems. A copper-clad kapton strip, permitting the application of voltage, extends from the electrode, offset with respect to the GEM contacts. A fiberglass frame, $3 \mathrm{~mm}$ thick and $7 \mathrm{~mm}$ wide, glued over the electrode defines the sensitive volume of the detector; a groove in this frame distributes the gas input through narrow holes along two sides of the chamber. The gas output is made with a single, larger hole on the opposite

\footnotetext{
4 For an initial part of the production, due to a mistake in the design, some gaps were larger, up to $500 \mu \mathrm{m}$ : their effect is discussed later.
} 
corner on the lower honeycomb plate. To keep the material budget low, the GEM electrodes are supported by narrow ( $7 \mathrm{~mm}$ wide, $2 \mathrm{~mm}$ thick) fiberglass frames, to which the foils are glued in sequence. To avoid deformations of the structure, the stretching force applied to the foils during assembly is moderate and not sufficient to guarantee uniformity in the gaps, particularly taking into account the strong electrostatic attraction between electrodes. We have therefore used a grid of thin fiberglass strips, or spacer, within the active area, to maintain the gap; the spacer grid is machined from the same plate as the frame, and repeats the sector-beam killer pattern as seen in Fig. 13. The width of the spacer strips, $400 \mu \mathrm{m}$, introduces a small local efficiency loss, discussed later. To prevent the flow of epoxy towards the holes during assembly, the inner edge of the frame is grooved; small notches at the edges and on the strips improve the gas circulation between foils. After manufacturing on a precision milling machine, the grids are coated with a thin layer of two-component polyurethane to block residual spikes or broken fibers that could affect performance. Before assembly, the spacer frames are cleaned in a ultrasonic bath with demineralized water and HV tested between two metallic plates up to $5 \mathrm{kV}$ to verify their insulation.

\subsection{Two-dimensional readout board}

The two-dimensional printed circuit readout board (PCB) consists of two layers of perpendicular copper strips at $400 \mu \mathrm{m}$ pitch, the two layers being separated by $50 \mu \mathrm{m}$ thick kapton ridges (Figs. 14 and 15); each coordinate has 768 readout strips. The method of fabrication is close to the one used for GEMs: after photo-lithographic patterning one side with the set of wider strips, the $50 \mu \mathrm{m}$ kapton foil is glued face down to a thin support sheet (120 $\mu \mathrm{m}$ fiberglass). Next, the exposed side is patterned and etched with the perpendicular set of thin anodes; the polymer between the strips is then chemically removed in a solvent bath, exposing the lower strips. With $80 \mu \mathrm{m}$ wide strips on the top layer and $340 \mu \mathrm{m}$ on the bottom an almost equal charge sharing between the upper and lower coordinates is obtained (see section 4). To stabilize the surface and permit reliable wire-bonding to the readout electronics, as a final step all strips are chemically coated with a thin nickel layer and gold-plated. In this paper, we have conventionally named " $x$ " the coordinate measured by the set of thin $80 \mu \mathrm{m}$ strips, and " $y$ " the perpendicular one measured by the wider strips. 
To meet the overall position accuracy, we require a tolerance in the geometrical position of all strips of $\pm 100 \mu \mathrm{m}$; this is regularly checked on an optical comparator. For each foil, a pair of extreme points at each strip's end is measured along three lines (two at the edges and one in the middle of the foil); the average distances and their differences are recorded for off-line corrections. Figs. $16 \mathrm{a}$ and $\mathrm{b}$ give the distribution of the distance between the first and last strip, measured on ten production read-out planes, along three reference lines both for the upper and lower sets of strips. The distributions have a FWHM of $\sim 150 \mu \mathrm{m}$.

\subsection{Assembly of the chambers}

All GEM foils are individually inspected and tested before mounting. A first quality check is done at the production workshop and GEMs are accepted if the resistivity in air between the two sides exceeds 2 G $\Omega$ on each sector. The foils are then transferred to the assembly room; clean packing and storage are mandatory. Before a foil is used for production, an inspection is done by eye, followed by a more extensive optical survey performed under the microscope. To check the uniformity of the holes' diameter, the foils are laid over a light table, and pictures of transmitted light are taken with a digital camera. The presence of a few defects (mainly local absence of metal and underlying kapton) is recorded, and, if they are only a few, the foil is accepted, as this has been found to have no consequences in the operation. The high voltage quality is controlled regularly, before mounting and at different steps during the construction, placing the foils or the partly assembled chamber inside a clean Plexiglas box with electrical feed-through permitting to apply voltage to each GEM segment through $100 \mathrm{M} \Omega$ protection resistors (Fig.17). The box is flushed for several hours with nitrogen to reduce the moisture level before testing; the voltage is then slowly increased with continuous monitoring of the leakage current. In case of discharges, the rampup is suspended, and resumed after a few minutes, in a procedure usually referred to as "training". On raw foils, the acceptance requirement is a leakage current less than $5 \mathrm{nA}$ at $600 \mathrm{~V}$ on each sector, all others being grounded. A longer training procedure often permits to reach these performances on initially weak sectors; we have observed however that such sectors may show problems during long-term chamber operation. In the subsequent steps of assembly, the test voltage is reduced to $550 \mathrm{~V}$ and applied to all sectors; the 
requirements are then a leakage current per sector lower than $5 \mathrm{nA}$ and no discharges for more than two minutes ${ }^{5}$. In some cases, a second run of professional cleaning of a raw GEM with a weak sector permits to recover the foils. In a few occurrences, the acceptance criteria could not be met and the foil was removed and replaced.

A PCB is validated for chamber production if it satisfies the geometrical tolerance criteria mentioned above, and does not have shorts between strips. The insulation between strips is checked before assembly with simple contact tools containing a conductive elastomer strip, hard-pressed on the edges of the boards to make electrical connection to all or part of the strips in a plane. With these tools, one can find and localize shorts between the two coordinates; for testing adjacent strips, two smaller contact tools are used. If a short is detected, it is sometimes possible to remove it by additional cleaning ${ }^{6}$.

Manufacturing of the chamber is entirely carried out in a class 1000 clean room, with controlled temperature and low moisture levels $(\sim 40 \%)$. To prevent organic pollutions, facial masks and gloves have to be worn. A thick aluminum template with a matrix of pins inserted on precision-drilled holes is used to position the various frames during assembly; parts are kept in place with vacuum. For all gluing we have used Araldite 103 (see Table 1), because of the good electrical behavior, coupled with convenient handling properties (density and viscosity). To apply the epoxy to the frames, a small rolling wheel is mounted on a moveable carriage, positioned in front of the frame sides (Fig. 18). To speed up polymerization, the assembly is heated to $35-40{ }^{\circ} \mathrm{C}$ in an open flow of nitrogen to avoid concentration and deposition of vapors. After each gluing step, the HV behavior of the GEM is tested, applying the quality selection criteria specified above.

The steps for assembling a chamber are as follows. The construction begins pasting the drift electrode foil to the smaller honeycomb plate. The first fiberglass frame, $3 \mathrm{~mm}$ thick (drift gap), is glued over the plate using the reference pins to guarantee mechanical positioning. The GEM foils are slightly stretched and taped on a transfer frame, larger than the final detector. Glue is applied to the outer $4 \mathrm{~mm}$ of the frames using the guided roller, previously wetted with epoxy spread on a clean plastic foil. The foils are then

\footnotetext{
${ }^{5}$ The highest operating voltage applied to a GEMs is around $400 \mathrm{~V}$.

${ }^{6}$ In a few cases, a short between strips was found after completion of the chamber. It could be eliminated applying briefly a low voltage, high current source between the strips.
} 
positioned on the template with the transfer frame edges weight-loaded to keep the tension during curing. Special care is taken to prevent epoxies to diffuse into the GEM holes; it has been found indeed that, even using certified insulating glues, this can substantially increase the leakage current, possibly because of insufficient polymerization or moisture inclusion ${ }^{7}$. During the procedure, the inner part of the detector is always protected against glue droplets by a clean, thin polymer foil cut to size. The three GEM foils are mounted in sequence following the described procedure. The twodimensional readout circuit is separately pasted to the larger honeycomb plate, and then glued under load onto the stack of drift and GEM foils, making use of the alignment pins. To ensure a complete polymerization of the epoxy, each chamber is put in an oven heated at $40{ }^{\circ} \mathrm{C}$ for at least 12 hours; to avoid pollutions due to outgassing, nitrogen is continuously flown through the inlet of the chamber with the output open to air. As a final step, to prevent micro-leaks, the edges of the chamber are sealed with a thin layer of a transparent silicone resin with very good dielectric rigidity (see Table 1). After performing leak checks and if necessary local resealing, the detector is ready to receive electrical connections for final testing.

\section{DETECTOR COMPLETION AND VALIDATION}

\subsection{High Voltage distribution and test}

Before connecting the HV distribution boards, a final test of individual sectors in each chamber is performed with the standard gas filling of $\mathrm{Ar} / \mathrm{CO}_{2}$ $(70 / 30)$. The requirement is for each sector to hold $550 \mathrm{~V}$ with a leakage current not exceeding $5 \mathrm{nA}$. If the detector passes the test, the $\mathrm{HV}$ distribution boards are mounted; these circuits contain the resistive voltage divider used to apply the required voltage to all electrodes through individual $10 \mathrm{M} \Omega$ protection resistors (Fig.19). A remotely controlled switch permits to decrease the potential of the central sector in the last GEM (closer to the PCB) by around $200 \mathrm{~V}$; this results in complete insensitivity of the central region to the beam. Based on the results of the discharge studies, slightly offset values of the main chain resistors are used to impart higher (lower) gain of the first (last) GEM. Typical values of voltage and fields for a chamber operated at a total effective gain close to $10^{4}$ are given in Table 3 . In the case of a temporary

\footnotetext{
${ }^{7}$ We suspect a separation of the epoxy components by capillarity in the holes.
} 
or permanent short in one of the sectors, voltages applied to other electrodes are slightly modified; an example is shown by the rightmost columns in the table; although the voltage sharing is modified, the sum decreases by only 15 $\mathrm{V}$, with a gain reduction $(\sim 25 \%)$ that can be easily corrected by a small increase of the overall operating voltage. Due to the dispersions in the GEM gains and resistor values, exact values change slightly from chamber to chamber (see the next section).

The HV boards are manufactured on $200 \mu \mathrm{m}$ thick fiberglass, preassembled with resistors, cleaned and coated with an insulating layer of $\mathrm{HV}$ sealant at the CERN-EST-SMD workshop. Before mounting, the boards are tested up to $5 \mathrm{kV}$ and the value of each individual resistor is verified and recorded to allow calculation of the effective voltages applied. To establish electrical contacts, the kapton lips protruding from the various electrodes are soldered to pads on the board. To verify electrical connections, and to detect the presence of external weak points, a voltage largely exceeding the operational is applied with the chamber filled with pure $\mathrm{CO}_{2}$. External hot spots, if any, are found by flushing briefly with argon and resolved by additional coating. At this stage the chamber is completely gas and $\mathrm{HV}$ certified, ready for final laboratory testing.

\subsection{Laboratory performance tests}

The final electronics readout circuit, requiring complex gating functions and external triggering, is not suited for the survey of the gain performance of a detector. The read-out strips on each chamber are therefore temporarily bonded to terminating printed circuit boards, providing access to the signal in groups of strips. In order to check the isolation between adjacent strips, the board provides separate contacts for odd and even strips, in groups of 16: shorts, due to residual dust or dirt introduced during assembly, can then easily be detected and eliminated with the brief application of a low voltage, high current source. A pair of low noise charge-sensitive amplifiers coupled to ADCs permit then recording of the signals on two groups at the time, normally corresponding to perpendicular coordinates in front of a source. To avoid cross talk and charging-up problems, all groups are grounded through resistors.

Each assembled and electrically connected detector is left for several hours on the operating gas flow (argon- $\mathrm{CO}_{2}$ 70-30) before application and 
gradual increase of the voltage, with continuous monitoring of the leakage current. At any sign of discharges, ramping of the voltage is temporarily suspended, and resumed after some time. Following this training procedure, the chamber can be brought up to nominal voltage and is very stable thereafter.

For a systematic mapping of the gain, each chamber is mounted on a mechanical displacement table in front of a $9 \mathrm{keV}$ collimated X-ray generator. A precise measurement of gain, indispensable to correlate laboratory and beam data, is done measuring the collected current at a known radiation flux ${ }^{8}$ [12]. An example of gain measured in two positions of a chamber having extreme values is shown in Fig. 20; the difference ( 30\%), measured from the current, is consistent with the one deduced from the pulse height. Making use of a multiple-hole reference board, placed in front of the detector, pulse height spectra are recorded for each chamber on 16 points over the active area, and on both projections. Examples of pulse height spectra are given in Fig. $21 \mathrm{a}$ and $\mathrm{b}$, respectively for the $\mathrm{x}$ and $\mathrm{y}$ set of strips. The energy resolution, $\sim 23 \% \mathrm{fwhm}$, is dominated by noise, due to the large capacitance of the group of strips (about $400 \mathrm{pF}$ ). A fit to the spectra permits to obtain the gain and energy resolution map for each chamber, recorded in view of corrections to the data in beam running conditions.

Fig. 22 and 23 provide, for a typical detector, histograms of the normalized gain distribution and of the energy resolution. The gain variations, $\sim 8 \% \mathrm{rms}$, are imputed to the cumulative effect of variations in the GEM hole diameters. The ratio of the charge collected on the two projections, or relative charge sharing, is given for the same chamber in Fig. 24, and is roughly equal to one, with a variance FWHM of $7 \%$. Small differences in average gain of the chambers due to the tolerances in the geometry and variations of resistor values in the dividers are compensated with the application of a slightly different operating voltage, adjusted in order to obtain a minimum nominal gain of 8000 in any position. The distribution of required voltage, measured on eight detectors, is shown in Fig. 25; the empty (grayed) histogram corresponds to the point of minimum (respectively maximum) gain in each detector.

\footnotetext{
${ }^{8}$ Measured on the anode strips, the gain corresponds to the value seen by the electronics; because of charge losses in the structure, this effective gain is smaller than the real one.
} 


\subsection{Readout electronics}

The final readout electronics makes use of the APV25-S0 chip, a 128 channel amplifier-shaper ASIC with an analogue pipeline 192 cells deep for each input channel [13]. The chip is fabricated in a 0.25 micron CMOS process to take advantage of the radiation tolerance, low noise and power consumption intrinsic to the technology. Originally designed for the readout of silicon micro-strip detectors, the chip does not have input protection against overload currents coming from the detector. Together with the efforts in reducing the discharge probability and energy, we have added an external input protection circuit to maximize the reliability of the system. It consists of a pair of inverted high-speed signal diodes (BAV99) to ground, and a decoupling capacitor $(220 \mathrm{pF})$ to avoid leakage currents into the amplifier, as shown in Fig. 26. The potential of the anode strips is set close to ground by the leakage current of the unbiased diodes. Input signals are pre-amplified and shaped by a CR-RC filter with 50 ns peaking time. Note that only the fast electron charge is detected on the readout strips, without slow ion components, a unique property of the GEM concept that is essential for good pile-up rejection.

The amplifier outputs are sampled at a rate of $40 \mathrm{MHz}$ and stored in the pipeline allowing for a maximum of $4.8 \mu$ s for external trigger selections. When a trigger is received, data in the corresponding pipeline columns are readout to a FIFO buffer. In total, up to 32 samples can be buffered in this FIFO awaiting readout. The so-called multi-mode operation of the chip is used, where three consecutive samples of a signal are stored for each trigger, the third sample corresponding to the peak of the input signal, and the second and first samples on its raising edge $25 \mathrm{~ns}$ and $50 \mathrm{~ns}$ earlier. This permits extracting accurate timing information of the signal, a feature very important to reduce pile-up in high-intensity beams. The samples are then read out separately through an analogue multiplexer operating at $20 \mathrm{MHz}$, its output being converted into a differential signal by the output buffer of the chip with an analogue gain of $1 \mathrm{~mA}$ for 25000 input electrons (the typical signal for a minimum ionizing track). After $60 \mathrm{~cm}$ of high-density flat cable, the multiplexed data from each chip are transmitted by a differential voltage amplifier through $2 \mathrm{~m}$ of twisted pair flat cable to the analog-to-digital converter (ADC). A 10-bit ADC samples the incoming, multiplexed data 
stream of one chip at $20 \mathrm{MHz}$ with one ADC channel corresponding to an input charge of $\sim 300$ electrons. After digitization, pedestal values are subtracted and a possible shift common to all channels in one chip, due to pickup noise in the detector, is corrected using one field programmable gate array (FPGA) for groups of six APV25 chips. If desired, the FPGA also performs zero suppression by sending out only those channels with amplitudes higher than a programmable multiple of the respective noise figure. For a detailed description of the algorithms developed for this purpose, as well as a full account of the readout system, refer to Ref. [14].

Data from the ADC module are then sent via a bi-directional optical fibre using the Hotlink protocol to a 9U VME module GeSiCA (GEM and Silicon Control and Acquisition). There, the data from up to 4 GEM detectors (48 APV25 chips) are buffered and merged before being sent, again via optical fibres using the S-Link protocol, to the DAQ computers. GeSiCA also encodes the trigger and clock signals received from the COMPASS Trigger Control System (TCS) [15] as well as slow control sequences for the ADC and APV25 chips, and sends them through the same Hotlink fibres to the front-end modules. The readout chain for GEM detectors in COMPASS is shown schematically in Fig. 27.

Printed circuit cards on the detector host three chips each and all ancillary components for the readout of 384 strips (one half of a coordinate); as seen in the picture of Fig. 28, they contain the input protection circuit (at $400 \mu \mathrm{m}$ pitch), followed by a thin glass pitch adaptor ${ }^{9}$ to bring the signal lines to the chip. The cards are fixed to the larger detector honeycomb plate and wire-bonded to the signal strips. Fig. 29 (full line) gives the estimated noise $\sigma_{\mathrm{N}}$ rms, given in number of electrons, for the APV25-S0 as a function of input capacitance; the points are experimentally measured for no input and two values of input capacitance corresponding to the narrow and wide strips in the COMPASS chambers.

\section{MISCELLANEOUS LABORATORY MEASUREMENTS}

In the course of the final development of the Triple-GEM detectors, several laboratory measurements and verifications have been performed on prototypes or final detectors; they are here briefly summarized.

\footnotetext{
9 Made on $300 \mu \mathrm{m}$ Schott D263 glass with $0.6 \mu \mathrm{m}$ wide aluminum strips.
} 


\subsection{Rate effects, charging-up}

Because of the small residual conicity of the GEM holed, dictated by the yield requirements discussed in Section 2.3, the electrodes are expected to experience a small gain increase due to charging-up during irradiation. As described in previous papers [16, 17], charges accumulate on the kapton surface until an equilibrium condition is reached, with no field lines left emerging from the walls. Due to the very high resistivity of the insulator, neutralization of the deposited charges takes much longer than charging up. Because of local variations in the diameter of the holes, the observed increase varies with position, but it has not been seen to exceed $30 \%$ in the worst case. As charging-up is rather fast, the procedure for the measurement is to set the voltage on the chamber and open the X-ray beam stopper after starting the recording of current in small time intervals. While the charging-up time constant depends on the source intensity, once saturation is reached, the pulse height is rate independent, as shown in Fig. 30. For this measurement, we have set the chamber at its nominal operating gain (8000). After charging-up, and in absence of radiation, the gain returns to its original value with a time constant of several days as shown in Fig. 31. The irradiation was resumed only for a short time (10 s) for each point at very low source intensity. Note that a moderate local increase of gain can only be beneficial for the operation, improving efficiency and localization accuracy; in the intense particle flux of the experiment, detectors quickly charge up at their plateau gain condition.

\subsection{Effects of sector boundaries and spacers}

The perturbing effect of sector separations and spacers has been investigated with a narrow collimated X-ray beam, moved in regular steps across the feature under study. Whilst the best information on charge losses and distortions can be obtained with individual strip readout, in the laboratory only signals on groups of strips could be recorded. The presence of a charge collection defect is then revealed by a local decrease in pulse height, current, energy resolution or counting rate. The intrinsic track width and electron diffusion can smear the primary ionization, partly obliterating the effects. In the sector boundary region, aside from the normal GEM charging up within the holes, discussed above, the narrow gap between sectors can also charge up, collecting ionization electrons. Equilibrium is reached when no field line enters the dielectric, with the effect of repelling electrons and 
gradually improve charge collection. Pulse height spectra, measured irradiating with a collimated X-ray beam the region of a sector boundary used in the final design of the chambers (200 $\mu \mathrm{m}$ wide), gradually evolve from rather poor, due to charge losses, to almost normal (Fig. 32). As shown in Fig. 33, providing the peak pulse height as a function of time, a fast gain increase presumably due to standard charging up is followed in this case by a slower rise towards saturation. Understandably, the recovery of collection efficiency results in a local distortion in the position measured through simple center of gravity algorithms; this has been confirmed in the beam runs, and will be discussed later.

The charge loss is more conspicuous close to a spacer, a physical obstruction, $\sim 400 \mu \mathrm{m}$ wide, presumably due to capture of part or all local charge; by construction, spacers always correspond to a sector boundary, so one can only measure the cumulative effect of the two. Fig. 33 shows the evolution in time of the pulse height spectra measured over a spacer; despite an increase of the average, spectra are rather poor and presumably result from the overlap of signals corresponding to fully and only partly collected charge. Since for a given insulating gap width one expects the charge loss to decrease with an increasing size of the primary ionization cloud, we have tried to increase diffusion operating the detector at lower drift field or lower $\mathrm{CO}_{2}$ content; in the range covered (limited by other factors) there was no noticeable improvement. More detailed information on the detection efficiency loss in the spacer's region will be discussed in the next chapter.

4.3 Discharge rate: influence of gain asymmetry and moisture

Exposed to heavily ionizing particles, gas detectors discharge at a value of gain much smaller than normal; this effect has been observed and extensively studied in a wide range of devices and conditions [17-19]. The introduction of the Gas Electron Multiplier as pre-amplifying device for other detector types has permitted to largely increase the maximum overall gain before discharges. We have investigated the effect of several parameters on discharge rate, exposing detectors to an alpha-emitting source $\left({ }^{220} \mathrm{Rn}\right)$ introduced with the gas flow. It should be emphasized that discharge probabilities obtained in such a way have only relative meaning, since absolute values depend on the ionization source. For the case of multi-GEM structures the discharge probability at a given value of total gain is strongly 
reduced by the application of asymmetric voltage values imparting larger gains to the first multiplier [7]. Fig. 35 shows the effect, measured on a production chamber; curves provide the discharge rate as a function of total effective gain for $0,18 \%$ and $39 \%$ voltage asymmetry, defined as the voltage difference between GEM1 and GEM3 divided by the voltage in GEM2. With the powering scheme adopted (Fig. 19 and Table 3), the asymmetry is $22 \%$ with a reduction of discharge rate, as compared to equal gain sharing, of more than two orders of magnitude.

A somewhat unexpectedly strong influence of discharge rate on the residual moisture in the gas has been found. This observation was made on a small double-GEM detector (10x10 $\mathrm{cm}^{2}$ active area), having a $5 \mu \mathrm{m}$ polymer window to permit exposure to an external alpha source; it was already suspected in previous work that water permeation through the thin window could affect the measurements [7]. The detector could be efficiently shielded against moisture intakes using a nitrogen-filled plastic bag wrapper. The water content in the gas outflow could be continuously monitored with a precision hygrometer and varied modifying the gas in the wrapper (mixtures of air with nitrogen) or by addition of sections of plastic tube in the gas inlet. Fig. 36 shows the discharge probability per alpha particle and as a function of effective gain for several moisture levels. It is not known if this effect is due to some gas process or to changes in the surface conductivity of the insulator. Whilst the intrinsic errors in this measurement are rather large, the strong dependence of discharge rate on moisture is apparent, and the observation should be considered as a warning against the reliability of measurements made in different conditions or detectors. We have verified that, with the use of metal tubing and at the normal gas flow $\left(\sim 60 \mathrm{~cm}^{3} / \mathrm{min}\right)$, the COMPASS detectors both in the laboratory and in the beam operate at a moisture level close to the minimum reached in the measurement ( $50 \mathrm{ppm})$.

\subsection{Gain dependence on pressure and temperature}

The gain in proportional counters depend, approximately through an exponential function, on the gas density, function itself of the ratio $\mathrm{T} / \mathrm{P}$ of absolute temperature and pressure. During long-term measurements, for example to study aging under irradiation, we have monitored temperature and pressure in order to establish the correlation with the value of gain; whilst the temperature in the laboratory was reasonably constant $\left( \pm 3^{\circ}\right.$, or 
$\sim 1 \%$ ), pressure could vary by as much as $5 \%$. The scatter plot in Fig. 37 correlates the value of gain $G$, measured at low rate and fixed voltage with the triple-GEM detector, to the ratio $\mathrm{T} / \mathrm{P}$. A best fit to the data with a function of the form $\ln G=A+B T / P$ gives for the slope parameter $B$ the value of 34.4 $\mathrm{K} / \mathrm{hPa}$. The dependence can be used in the experiment to actively correct the value of applied voltage in order to maintain constant gain in varying ambient conditions.

\subsection{Aging under sustained irradiation}

The relative immunity of GEM detectors to aging processes under heavy irradiation has been reported by many authors [12, 20-22]. Most of these measurements were made however with specially prepared, small size detectors taking precautions to avoid the use of polluting materials. For this work, we have irradiated a standard production triple-GEM detector, at an operating gain ( 8500) slightly higher than normal, for about a month with a strong flux of X-rays from a generator, continuously monitoring current and gain, both in the exposed region (an area of around $80 \mathrm{~cm}^{2}$ ) and outside. A detailed description of the experimental procedure and of the results is reported elsewhere [23]. Fig. 38 summarizes the results, after proper normalization (in particular removal of the pressure-temperature gain variations, see above). After a total collected charge exceeding $7 \mathrm{mC} / \mathrm{mm}^{2}$, no change of gain was detected; this corresponds to 7 years of operation in the experiment. Possible reasons for this excellent behavior, in cleanness conditions far from those required by other micro-pattern devices, might be the larger area available for polymer deposits, and/or the relative independence of the gain from the presence of a thin insulating layer on the electrodes. For a discussion see Ref. [23].

4.6 Mechanical distortions due to the gas flow

Installed in the experiment, the detectors are operated on an open gas flow, with pairs of chambers in series and rather long input and output pipes; the chamber itself represents an impedance for the flowing gas. Inserting output pipes of various diameter and length, we have changed the load, measuring both the overpressure as a function of gas flow directly at the output of the chamber and the inflation in its center with a micrometer. Fig. 39 shows the measured points; a straight line fit through the data, extrapolated to zero inflation, provides the pressure load of the chamber 
itself, 40 Pa. To avoid excessive deformation, the impedance of the output pipes has obviously to be kept as small as possible.

\section{BEAM TESTS}

Prototypes of the COMPASS detectors have been tested, at various stages of their development, both in the laboratory and with beam exposures $[10,24]$. Here we describe only results obtained with production devices, equipped with the final electronics. Three detectors, code-named TGEM9, 10 and 11, have been installed in the T11 beam line at CERN, and exposed during several weeks to a $3.6 \mathrm{GeV} / \mathrm{c}$ non-separated charged particles beam with an intensity of $\sim 10^{6}$ particles per $200 \mathrm{~ms}$ spill. The area of the beam was around $100 \mathrm{~cm}^{2}$. Only two chambers (TGEM 10 and 11) were fully equipped with readout electronics, the third having only one coordinate read out. Two upstream scintillation counters, covering an area of $10 \times 10 \mathrm{~cm}^{2}$, and a downstream narrower $\left(5 \times 2 \mathrm{~cm}^{2}\right)$ counter were used for triggering. For part of the runs, a silicon micro-strip detector ${ }^{10}$, with an active area of $5 \times 7 \mathrm{~cm}^{2}$ was used to obtain a precise measurement of the beam tracks in one point. Both silicon and gas detectors data were recorded making use of the COMPASS data acquisition system [25].

For each event, the system records the input charge on each strip, integrated over three adjacent time slots of 25 ns. Raw data undergo a standard procedure of pedestal and common mode subtraction, removing both DC offsets and large, externally induced baseline shifts. A cluster finding algorithm then tags the channels with a content exceeding a preset value of threshold (typically $3 \sigma_{\mathrm{N}}$, the rms of the noise). Channels with the highest content are selected, and a cluster is defined as the group of adjacent strips with a content exceeding threshold. A cluster is validated if its total charge exceeds $5 \sigma_{\mathrm{N}}$. For the analysis, only the content of the highest (usually the third) digitized time bin has been considered, and no timing cuts applied. The algorithm recognizes multiple clusters on a coordinate if separated by a significant local minimum. For the recognized clusters, the analysis program calculates the center of gravity, representing the space coordinate, the width and the total charge.

\footnotetext{
10 Provided by the collaboration
} 
Fig. $40 \mathrm{a}$ and $\mathrm{b}$ show, for a typical run, the cluster charge distribution for the $\mathrm{x}$ and, respectively, the $\mathrm{y}$ coordinate, recorded on TGEM11 at the nominal operating voltage $\left(\mathrm{V}_{0}=-4 \mathrm{kV}\right)$. The horizontal axis is given in units of ADC counts ${ }^{11}$; a threshold has been set at $3 \sigma_{\mathrm{N}}$ for each channel. Fig. 41 provides the distributions of cluster size, defined as the number of adjacent strips above threshold, for the two projections; both the FWHM and the width above the threshold baseline are provided. As it can be seen, the majority of events are recorded on two or more strips, therefore permitting an accurate estimate of the coordinate by charge interpolation. The scatter plot in Fig. 42 shows the correlation between the cluster charges measured on the two projections; the ratio of charges has a quasi-gaussian shape, centered around 1 , with a width of $9.2 \%$ rms (Fig. 43). The very good correlation offers a powerful way to resolve ambiguities in case of multiple tracks (see later).

Using as reference the silicon micro-strip, we have measured the efficiency of each chamber, as a function of applied voltage; efficiency is here defined as the presence of a cluster with a center of gravity within $\pm 400 \mu \mathrm{m}$ of the predicted position. The hit in the silicon, aligned with the one reconstructed in one chamber, operated at fixed conditions, defines a "road" for the search of a point in the chambers under study. The efficiency plateau and signal/noise ratio measured for TGEM11 are shown in Fig. $44 \mathrm{a}$ and $\mathrm{b}$ ( $\mathrm{x}$ and y coordinates, respectively). One can see that the efficiency plateau for minimum ionizing particles, perpendicular to the chamber, is reached at an overall voltage of - $4075 \mathrm{~V}$; with reference to the measured absolute effective gain for the same chamber (Fig. 20), this corresponds to a minimum gain of around 8000 , or $\sim 4000$ for each coordinate. The signal/noise, defined as the ratio of total cluster charge to the cluster noise (rms) is around 18. Note that, integrated over a large area that includes sector boundaries and spacers, the efficiency does not reach its full value; the local losses due to the various features are estimated to be about $2 \%$. The irradiated area of the chambers was partly covered by the beam killer $(5 \mathrm{~cm} \varnothing)$; this region could be activated by remotely controlling its potential. In Fig. 45 two-dimensional plots of the reconstructed coordinates in TGEM 11 are given for active (a) and inhibited center (b). The number of detected tracks in the beam killer region (mostly

\footnotetext{
11 As indicated previously, one ADC channel corresponds to an input charge of around 300 electrons
} 
due to accidentals) is negligible. The "shadow" effect of the spacer and sectors boundaries is clearly visible in the figures. A projection along one axis of the scatter plot gives the histogram shown in Fig. 46; the larger depletion on the right side corresponds to a spacer, whilst the narrower near the center is due to a sector boundary. The noticeable increase of counts at the two edges of each depletion is suggestive of the fact that part of the missing tracks are actually reconstructed with a small lateral displacement, a consequence of one or more missing signals on the strips facing the perturbation. A reconstruction algorithm taking this effect into account is under development. As mentioned before, the relatively wide perturbation around the sector boundary was due to an erroneously large separation $(500 \mu \mathrm{m})$ in the early production, and has been corrected since. A plot of integral efficiency as a function of position along one coordinate clearly shows the locality of the losses (Fig. 47). In the active area, the efficiency is close to $99 \%$.

Figs. $48 \mathrm{a}$ and $\mathrm{b}$ give the distribution of the deviation, in TGEM11, of the measured coordinate from the one computed from the points given by the silicon micro-strip and TGEM10, respectively for the $\mathrm{x}$ and $\mathrm{y}$ coordinate. A gaussian fit to the distributions provides a standard deviation around $70 \mu \mathrm{m}$; assuming the silicon accuracy to be $15 \mu \mathrm{m}$, the two chambers to be equal in response, and allowing for the increased multiple scattering, this agrees with the $\sim 40 \mu \mathrm{m}$ rms space accuracy previously obtained with prototypes [26].

As mentioned, the input charge is recorded in three consecutive bins, at $25 \mathrm{~ns}$ intervals, timed to provide three measurements on the raising edge of the signal. The time of the event with respect to the trigger can be estimated fitting the three measured samples to the (known) average pulse rise time for minimum ionizing tracks crossing the detector. For the present runs, however, the phase of the master clock in respect to the trigger was not measured, producing an additional spread. A scatter plot of the charge ratio between second and third sample as a function of the ratio between first and second clearly shows the tail of events corresponding to a wrong clock phase (Fig. 49). Removing the tail events, the projection of the distribution on the time axis provides the histogram shown in Fig. 50; a gaussian fit to the data yields a time resolution of $15.2 \pm 0.1 \mathrm{~ns}$ rms. This is obviously the result of a convolution of physical and electronics effects, and does not correspond to the intrinsic resolution of the detector, measured to be, in similar conditions, 
around $7 \mathrm{~ns}$ rms [26]. A measurement of the clock phase in respect to the trigger is being implemented in the experiment and will remove the ambiguity.

Due to the moderate beam intensity during the test period, we could not obtain a sizeable sample of multiple-track events. Using a classic software procedure, we have superimposed several single-track events and analyzed the results. For a single chamber, and in absence of external constraints, the almost equal charge sharing between coordinates offers a powerful way to resolve ambiguities. A preliminary result of this study is shown in Fig. 51, providing the probability of correctly coupling the two coordinates in all tracks, as a function of their number, both for single hits and for the full event. For example, $65 \%$ of the tracks are well reconstructed for events with multiplicity four, a rather unique and powerful feature of the 2-D projective readout. Obviously, tracking between detectors, and in particular within the two rotated chambers in a pair, will help removing most of the ambiguities.

\section{CONCLUSIONS AND SUMMARY}

A high-rate tracker making use of triple-GEM gas detectors is being commissioned for the COMPASS experiment; at the time of writing (November 2001) 14 chambers are installed and operational. The final set-up will mount a total of 20 modules, each with an active area of $\sim 1000 \mathrm{~cm}^{2}$ and fast two-dimensional coordinate read-out. In an extended set of laboratory and beam tests we have demonstrated the excellent performances and operating robustness of the detectors. With position accuracy around $40 \mu \mathrm{m}$ rms, excellent multi-track resolution, rate capability in excess of $10^{5} \mathrm{~Hz} \mathrm{~mm}^{-2}$ and lifetime exceeding 5 years of operation in the harsh radiation environment, the detectors are expected to effectively contribute to the success of the experiment. 


\section{ACKNOWLEDGEMENTS}

The present work would not have been possible without the dedicated help of several CERN support groups. In particular, we would like to thank M. Delattre, A. Domeniconi and D. Lacroix (EP-TA1) for the mechanical design of the detectors, M. Sanchez, J. Alves (EST) for the design and manufacturing of the electrodes, A. Honma, I. Mc Gill and K. Mühlemann for the wire bonding artwork. R. Hammastrom (CERN), G. Hall and M. Raymond (Imperial College London), L. Schmitt (TU München) have provided invaluable help in finalizing the readout electronics. A. Bressan (INFN Trieste) participated to the initial design of the detector and preliminary data analysis. The contributions of H. W. Siebert, J. Pochodzalla and $\mathrm{Yu}$. Alexandrov to the design and installation of the detectors are here also acknowledged. 


\begin{tabular}{|c|c|c|}
\hline Material & Details & $\begin{array}{l}\text { Supplied or } \\
\text { manufactured by }\end{array}$ \\
\hline Assembly epoxy & ARALDIT AY103 + HD991 (ratio 10:4) & Cyba-Geigi \\
\hline $\begin{array}{l}\text { Frame \& spacer } \\
\text { conditioning }\end{array}$ & $\begin{array}{l}2 \text { component polyurethane } \\
\text { Nuvovern LW }\end{array}$ & $\begin{array}{l}\text { Walter Mader AG } \\
\text { CH-8956 } \\
\text { Killwangen }\end{array}$ \\
\hline $\begin{array}{l}\text { Honeycomb } \\
\text { sandwich } \\
\text { structure }\end{array}$ & $\begin{array}{l}2 \times 125 \mu \mathrm{m} \text { fibreglass- } \\
3 \mathrm{~mm} \text { honeycomb Nomex }\end{array}$ & $\begin{array}{l}\text { Stesalit } \\
\text { Socol F-1020 } \\
\text { Renens }\end{array}$ \\
\hline Shielding & Aluminium $(10 \mu \mathrm{m})$ & \\
\hline GEM foils & $\begin{array}{l}50 \mu \mathrm{m} \text { kapton, } 2 \times 5 \mu \mathrm{m} \text { copper, } 70 \mu \mathrm{m} \\
\text { holes at } 140 \mu \mathrm{m} \text { pitch }\end{array}$ & CERN-EST-DEM \\
\hline Drift & $5 \mu \mathrm{m}$ copper on $50 \mu \mathrm{m}$ kapton & CERN-EST-DEM \\
\hline Drift Frame & $3 \mathrm{~mm}$ thick Stesalit & CERN-EP-TA1 \\
\hline Spacers & Fibreglass grids $2 \mathrm{~mm}$ thick & CERN-EST-DEM \\
\hline Gas pipes & Polypropilene tube (3 $\mathrm{mm}$ diameter) & Angst-Pfister \\
\hline Gas outlet & Fibreglass + fitting & CERN-EP-TA1 \\
\hline Readout PCB & $\begin{array}{l}\text { Active area } 30.7 \times 30.7 \mathrm{~cm}^{2} \\
2 \times 768 \text { strips at } 400 \mu \mathrm{m} \text { pitch }\end{array}$ & CERN-EST-DEM \\
\hline HV boards & Fibreglass $400 \mu \mathrm{m}$ & CERN-EST-DEM \\
\hline $\begin{array}{l}\text { HV protection } \\
\text { and sealant }\end{array}$ & R4-3117 & Dow Corning \\
\hline
\end{tabular}

Table 1. Components used for the assembly of GEM detectors.

\begin{tabular}{|c|c|c|}
\hline Part & Details & $\%_{\text {oo }}$ of $X_{0}$ \\
\hline 3 GEMs & $\begin{array}{l}6 \times 5 \mu \mathrm{m} \text { copper [0.7] } \\
3 \times 50 \mu \mathrm{m} \text { kapton [0.7] }\end{array}$ & $\begin{array}{r}1.68 \\
0.42 \\
\text { TOTAL: } 2.10\end{array}$ \\
\hline 1 Drift & $\begin{array}{l}5 \mu \mathrm{m} \text { copper } \\
50 \mu \mathrm{m} \text { kapton }\end{array}$ & $\begin{array}{r}0.35 \\
0.17 \\
\text { TOTAL: } 0.52\end{array}$ \\
\hline 3 Grid spacers & $3 \times 2 \mathrm{~mm}$ fibreglass [0.008] & 0.25 \\
\hline 1 Readout board & $\begin{array}{l}80 \mu \mathrm{m} \text { strips: } 5 \mu \mathrm{m} \text { copper }[0.2] \\
340 \mu \mathrm{m} \text { strips: } 5 \mu \mathrm{m} \text { copper }[0.85] \\
50 \mu \mathrm{m} \text { kapton }[0.2] \\
120 \mu \mathrm{m} \text { fibreglass } \\
60 \mu \mathrm{m} \text { epoxy }\end{array}$ & $\begin{array}{r}0.07 \\
0.26 \\
0.03 \\
0.62 \\
0.30 \\
\text { TOTAL: } 1.28\end{array}$ \\
\hline 1 Shielding & $10 \mu \mathrm{m}$ aluminum ( & 0.11 \\
\hline 2 Honeycombs & $2 \times 3 \mathrm{~mm}$ Nomex & 0.46 \\
\hline 4 Fiberglass foils & $4 \times 120 \mu \mathrm{m}$ fibreglass & $\begin{array}{l}2.47 \\
710\end{array}$ \\
\hline
\end{tabular}

Table 2. Material budget for a Triple GEM detector in the active area. For some components, the fractional filling factor is given in brackets. 


\begin{tabular}{|c|c|c|c|c|c|c|}
\hline ELECTRODE & $\begin{array}{l}\text { VOLTAGE } \\
\text { (V) }\end{array}$ & $\begin{array}{c}\text { FIELD } \\
(\mathrm{kV} / \mathrm{cm})\end{array}$ & $\begin{array}{l}\Delta \mathrm{VV} \\
(\mathrm{V})\end{array}$ & GAIN & $\begin{array}{l}\Delta \mathrm{V}^{\prime} \\
(\mathrm{V})\end{array}$ & GAIN \\
\hline DRIFT & -4100 & \multirow{2}{*}{2.49} & & & & \\
\hline GEM1 TOP & -3353 & & \multirow{2}{*}{410} & \multirow{2}{*}{50} & \multirow{2}{*}{392} & \multirow{2}{*}{34} \\
\hline GEM1 BOT & -2943 & \multirow{2}{*}{3.73} & & & & \\
\hline GEM2 TOP & -2196 & & \multirow{2}{*}{374} & \multirow{2}{*}{23} & \multirow{2}{*}{376} & \multirow{2}{*}{24} \\
\hline GEM2 BOT & -1822 & \multirow{2}{*}{3.73} & & & & \\
\hline GEM3 TOP & -1075 & & \multirow{2}{*}{328} & \multirow{2}{*}{8.5} & \multirow{2}{*}{330} & \multirow{2}{*}{9} \\
\hline GEM3 BOT & -747 & \multirow{2}{*}{3.73} & & & & \\
\hline PCB & 0 & & TOT 1112 & 9775 & 1098 & 7344 \\
\hline
\end{tabular}

Table 3: Voltages, fields and gains in typical operating conditions. The last two columns give values in the case of one shorted segment in the first GEM. The quoted values are numerical estimates; exact values vary from detector to detector, both due to geometry and to tolerances of the resistor dividers. 


\section{REFERENCES}

[1] COMPASS, Common Muon and Proton Apparatus for Structure and Spectroscopy, CERN/SPSLC 96-14 (1996).

[2] G.K. Mallot, Prog. Part. Nucl. Phys. 36 (1996) 39.

[3] B. Adeva and e. al, Phys. Rev. D60 (1999) 072004.

[4] M. Moinester, Pion polarizabilities and hybrid meson structure at CERN COMPASS, HEP-EX/0012063.

[5] P. Abbon, J. Ball, Y. Bedfer, C. Carasco, E. Delagnes, D. Durand, J.-C. Faivre, H. Fonvieille, A. Giganon, F. Kunne, J.-M.L. Goff, F. Lehar, A. Magnon, D. Neyret, E. Pasquetto, H. Pereira, S. Platchkov, E. Poisson, P. Rebourgeard, D. Thers, Nucl. Instr. and Meth. A461 (2001) 29.

[6] F. Sauli, Nucl. Instr. and Meth. A386 (1997) 531.

[7] S. Bachmann, A. Bressan, M. Capeàns, M. Deutel, S. Kappler, B. Ketzer, A. Polouektov, L. Ropelewski, F. Sauli, E. Schulte, L. Shekhtman, A. Sokolov, Discharge studies and prevention in the gas electron multiplier, CERNEP/2000-151 (11.12.2000) Subm. Nucl. Instr. and Meth. .

[8] M. Ziegler, P. Cwetanski, U. Straumann, A triple GEM detector for LHCb, LHCb TRAC 99-024 (1999).

[9] S. Bachmann, A. Bressan, B. Ketzer, M. Deutel, L. Ropelewski, F. Sauli, Nucl. Instr. and Meth. A461 (2001) 42.

[10] S. Bachmann, A. Bressan, B. Ketzer, M. Deutel, L. Ropelewski, F. Sauli, A. Bondar, A. Buzulutskov, L. Shekhtman, A. Sokolov, A. Tatarinov, A. Vasil'ev, Nucl. Instr. and Meth. A470 (2001) 548.

[11] R. Bouclier, M. Capeans, C. Garabatos, F. Sauli, K. Silander, Nucl. Instr. and Meth. A350 (1994) 464.

[12] S. Bachmann, L. Bressan, L. Ropelewski, F. Sauli, A. Sharma, D. Mörmann, Nucl. Instr. and Meth. A438 (1999) 376.

[13] M.J. French, L.L. Jones, Q. Morrissey, A. Neviani, R. Turchetta, J. Fulcher, G. Hall, E. Noah, M. Raymond, G. Cervelli, P. Moreira, G. Marseguerra, Nucl. Instr. and Meth. A 466 (2001) 359.

[14] I. Konorov and e. al, Design of a readout system for GEM and silicon detectors for COMPASS. In preparation.

[15] I. Konorov and e. al, The trigger control system of COMPASS. In preparation. 
[16] R. Bouclier, W. Dominik, M. Hoch, J.C. Labbé, G. Million, L. Ropelewski, F. Sauli, A. Sharma, G. Manzin, Nucl. Instr. and Meth. A396 (1997) 50.

[17] A. Bressan, M. Hoch, P. Pagano, L. Ropelewski, F. Sauli, S. Biagi, A. Buzulutskov, M. Gruwé, A. Sharma, D. Moermann, G. De Lentdecker, Nucl. Instr. and Meth. A424 (1999) 321.

[18] P. Fonte, V. Peskov, B. Ramsey, IEEE Trans. Nucl. Sci. 1998 (1999) 91.

[19] V. Peskov, P. Fonte, M. Danielsson, C. Iacobaeus, J. Ostling, M. Wallmark, Fundamentals of Gas Micropattern Detectors, LIP/01-05 (2001).

[20] J. Miyamoto and I.P.J. Shipsey, Nucl. Phys. B (Proc. Suppl.) 78 (1999) 695.

[21] J. Miyamoto and I.P.L. Shipsey, IEEE Trans. Nucl. Sci. NS-46 (1999) 312.

[22] S. Bachmann, A. Bressan, A. Placci, L. Ropelewski, F. Sauli, IEEE Trans. Nucl. Sci. NS-47 (2000) 1412.

[23] C. Altunbas, K. Dehmelt, S. Kappler, B. Ketzer, L. Ropelewski, F. Sauli, F. Simon, Aging measurements with the Gas Electron Multiplier (GEM), (2001) Subm. Nucl. Instr. and Meth..

[24] B. Ketzer, S. Bachmann, M. Capeáns, M. Deutel, J. Friedrich, S. Kappler, I. Konorov, S. Paul, A. Placci, K. Reisinger, L. Ropelewski, L. Shekhtman, F. Sauli, IEEE Trans. Nucl. Sci. NS-48 (2001) 1065.

[25] ALICE DATE User's Guide, ALICE 99/46 Int. Note (1999).

[26] A. Bressan, J.C. Labbé, P. Pagano, L. Ropelewski, F. Sauli, Nucl. Instr. and Meth. A425 (1999) 262. 


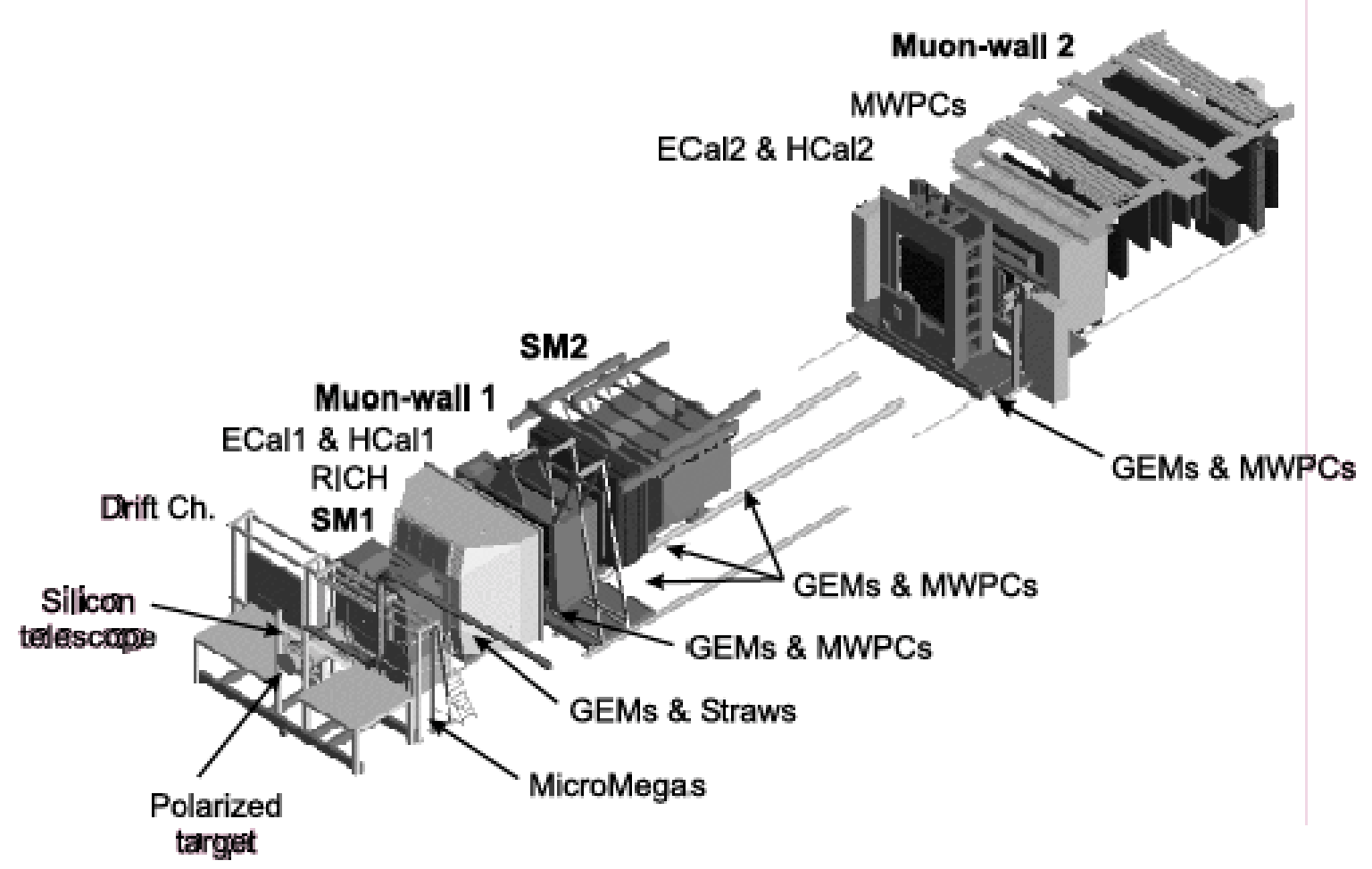

Fig. 1: Schematic layout of the COMPASS experiment.

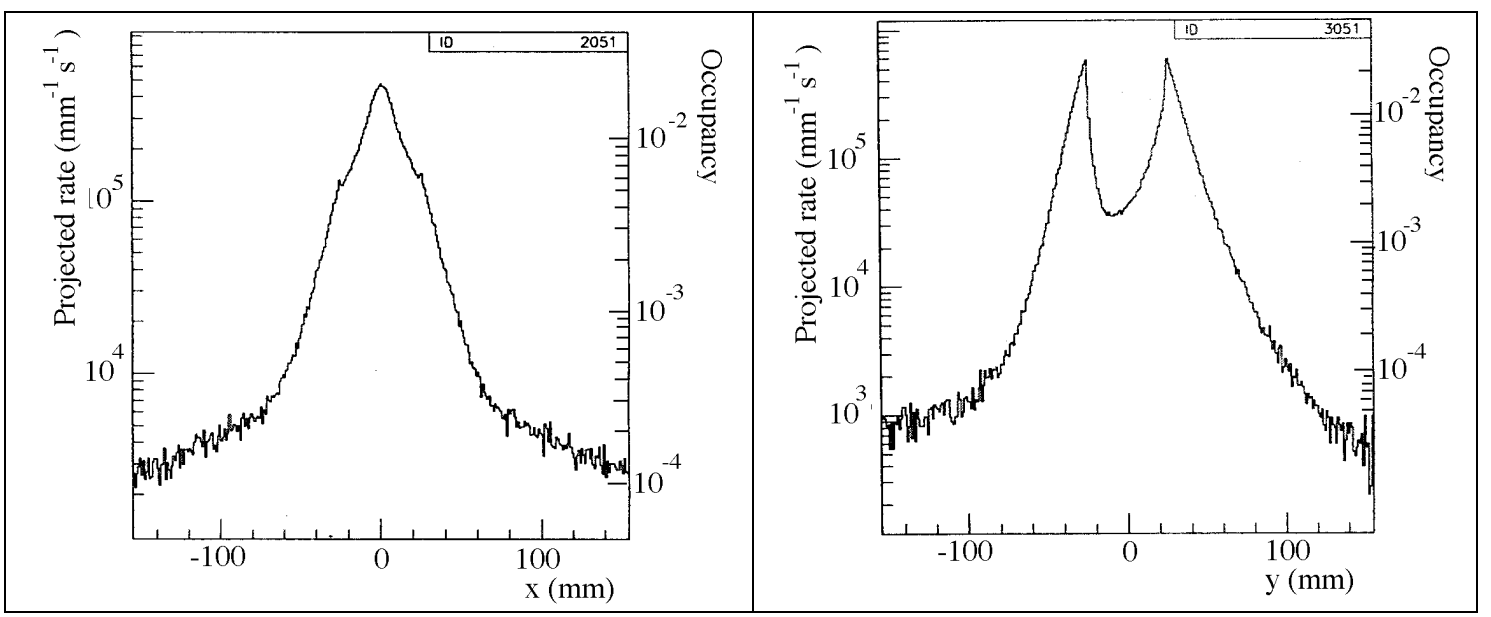

Fig. 2: Projected charged particle rates and occupancy computed at GEM station 4 (15 $\mathrm{m}$ from target) as a function of distance from the beam. 


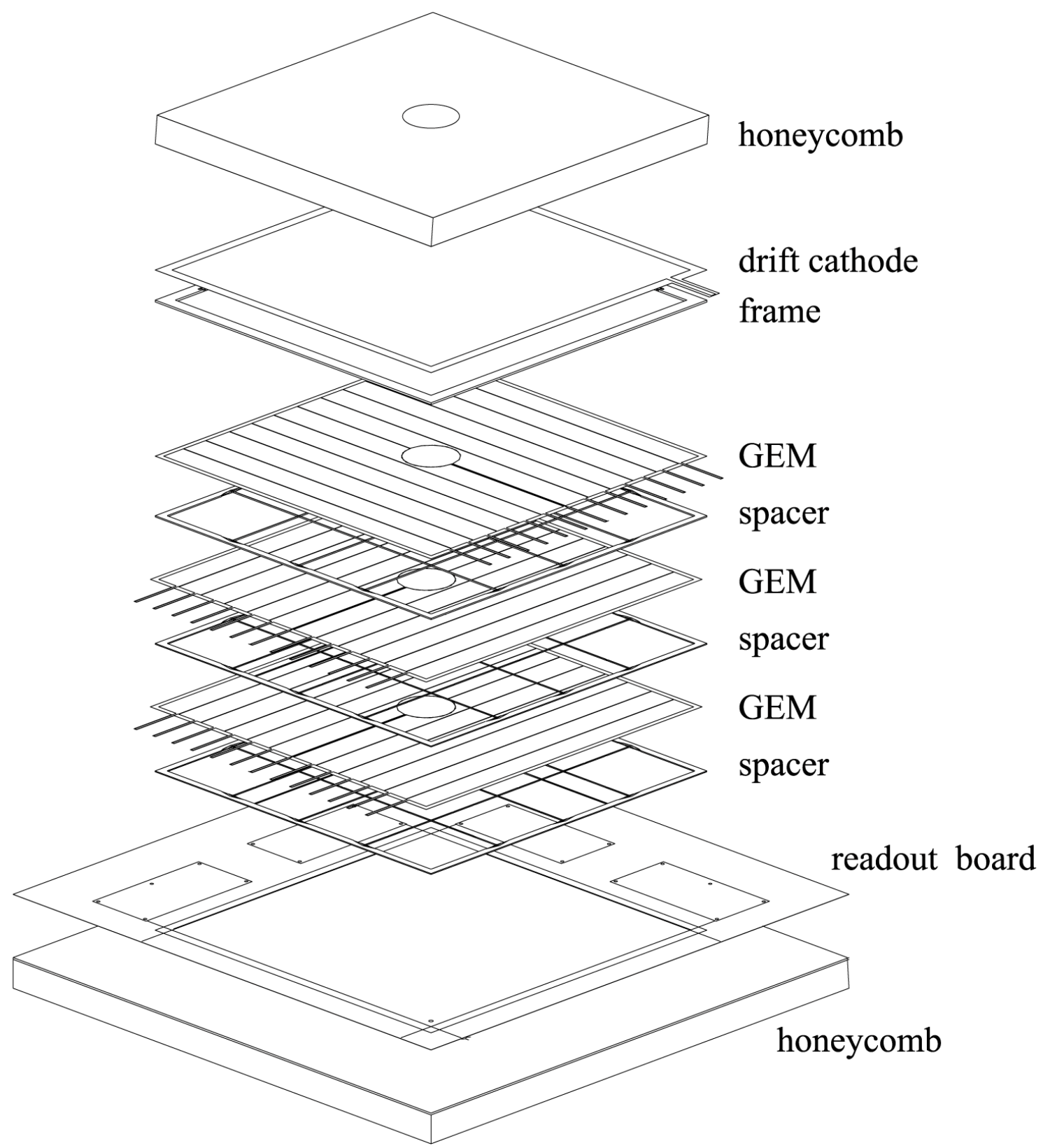

Fig. 3: Exploded view of the Triple-GEM assembly. 


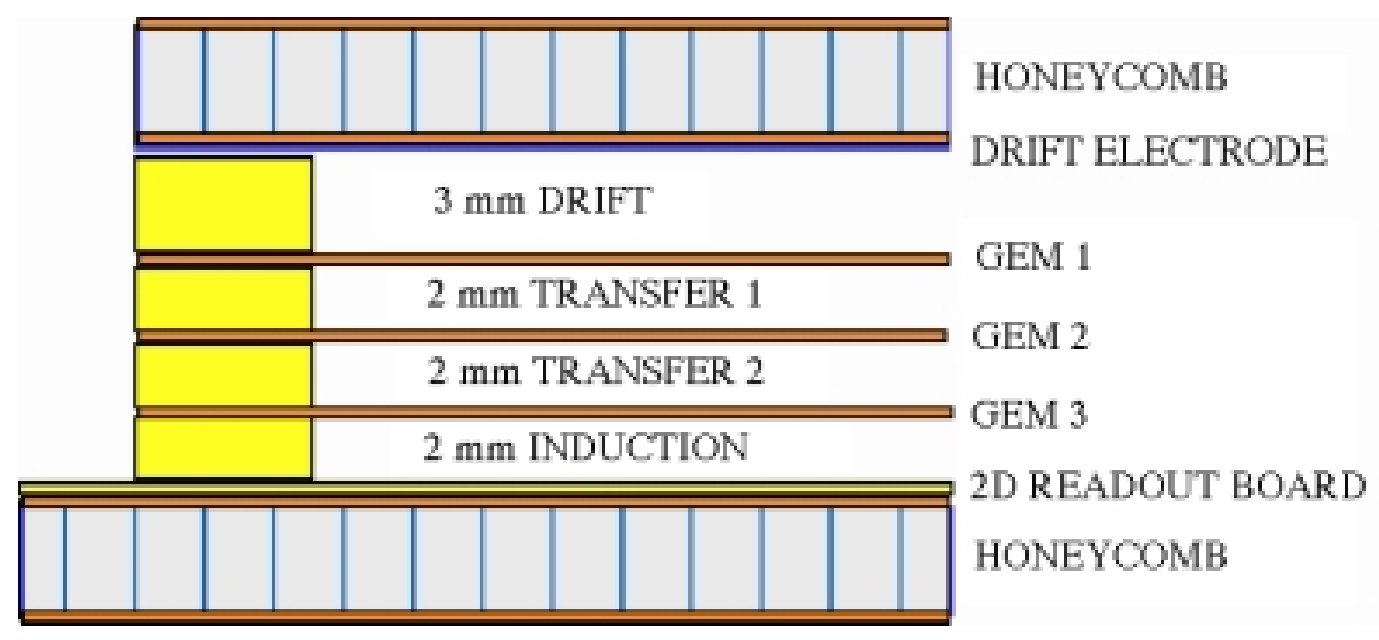

Fig. 4: Schematic cross section of the Triple-GEM detector.

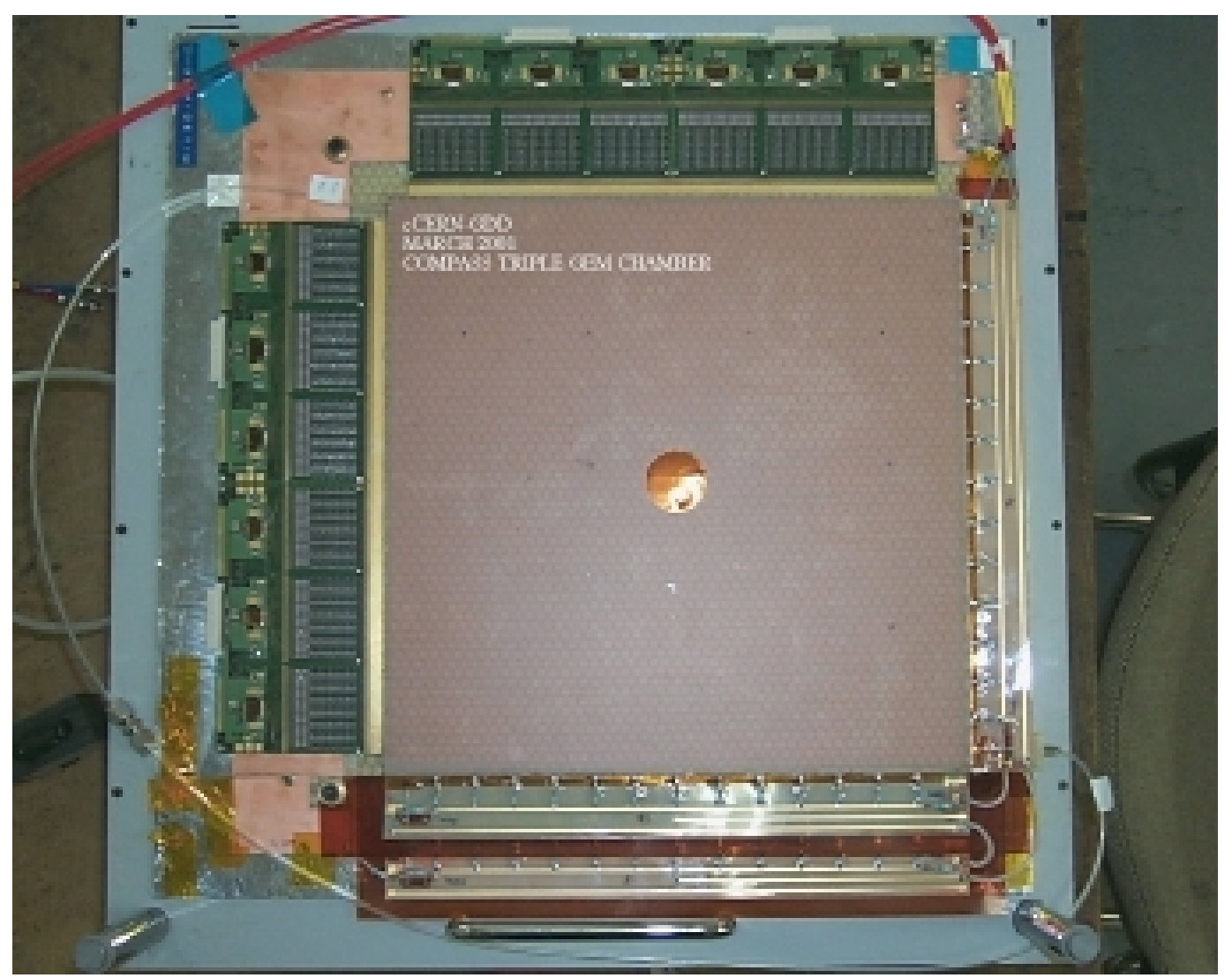

Fig. 5: A completed chamber, equipped with HV distribution and twodimensional read-out electronics. The active area is $\sim 31 \times 31 \mathrm{~cm}^{2}$. 


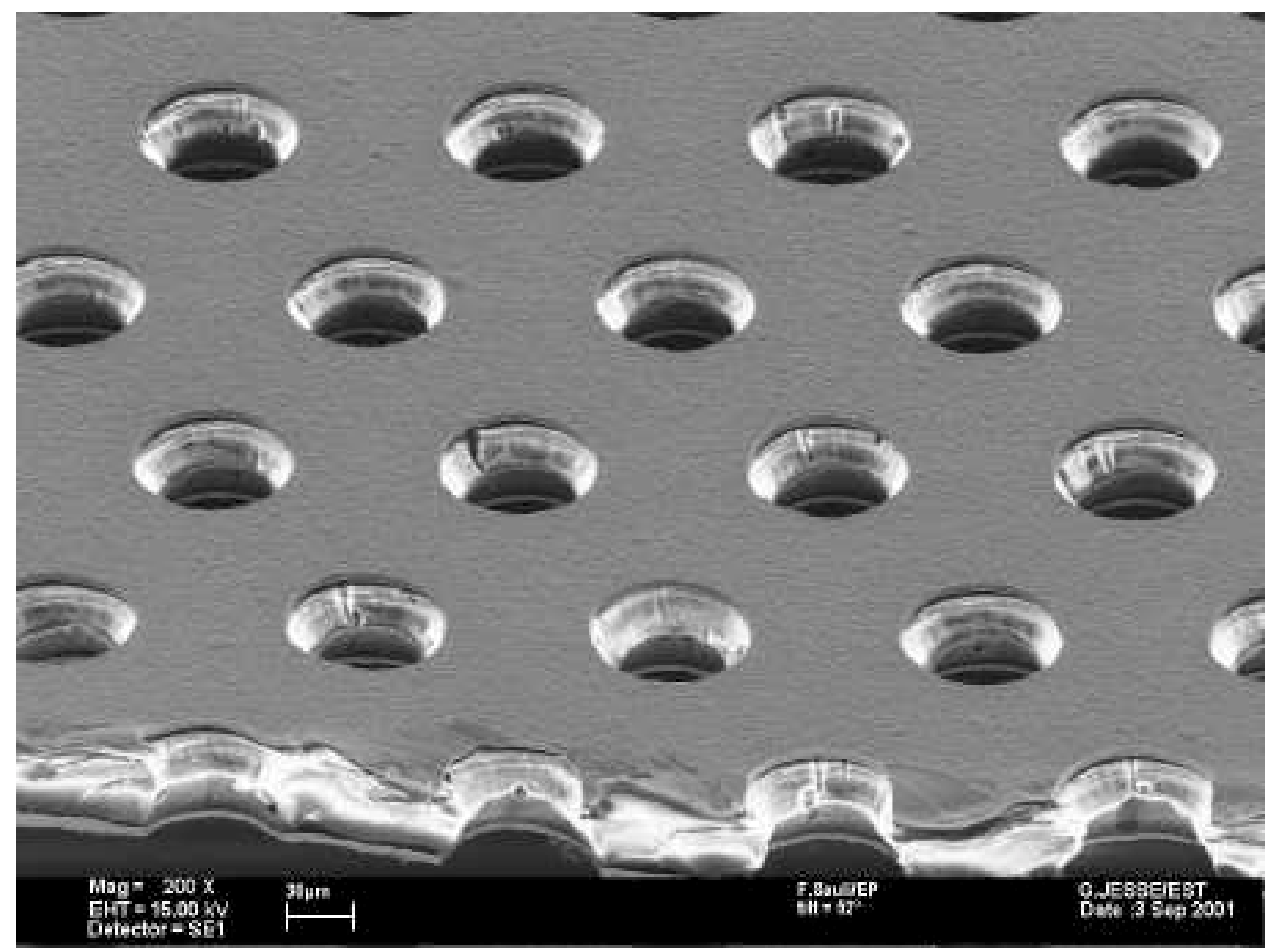

Fig. 6: Electron microscope picture of a GEM foil.

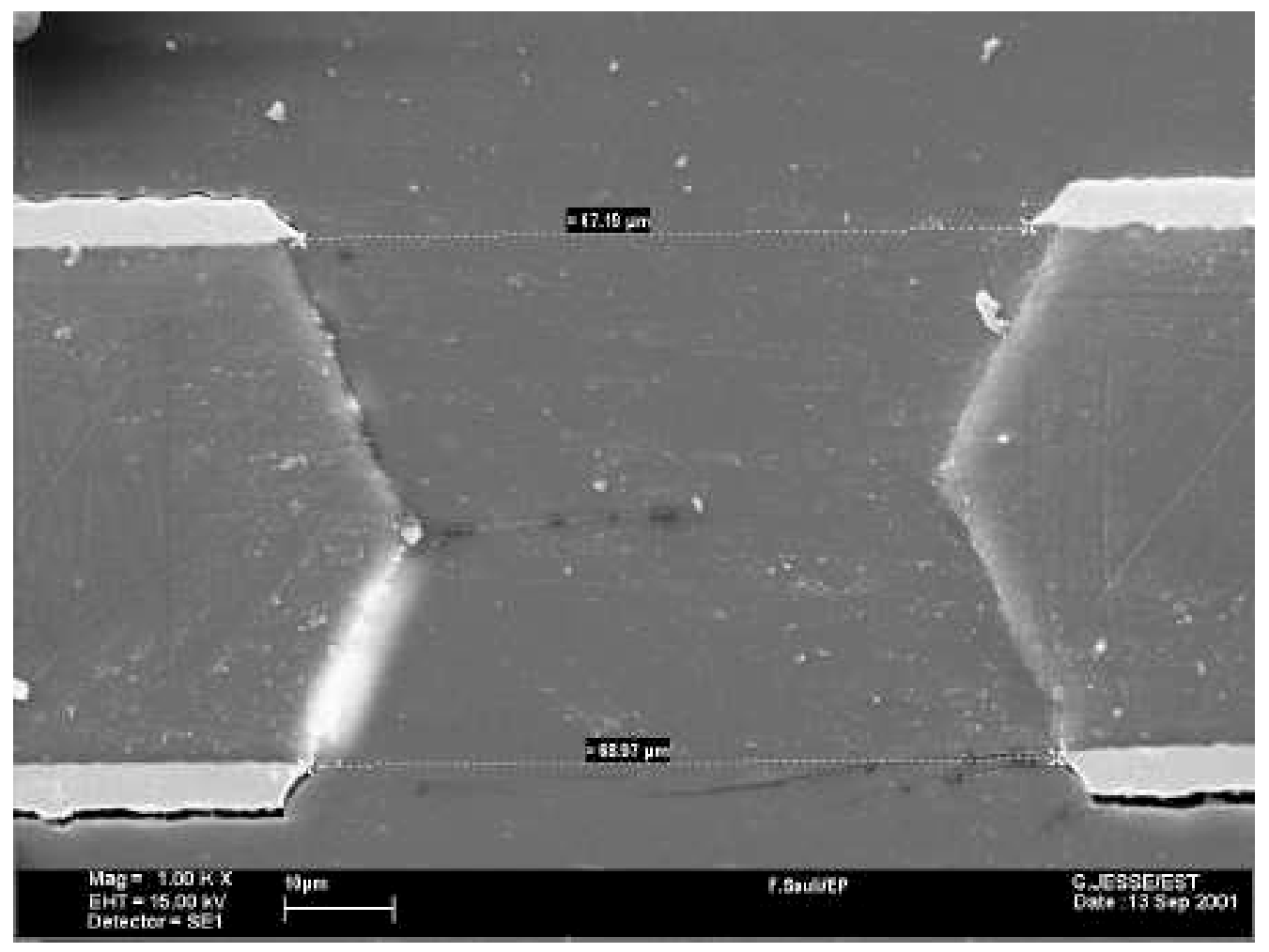

Fig. 7: Electron microscope view of a cross section through one hole. 


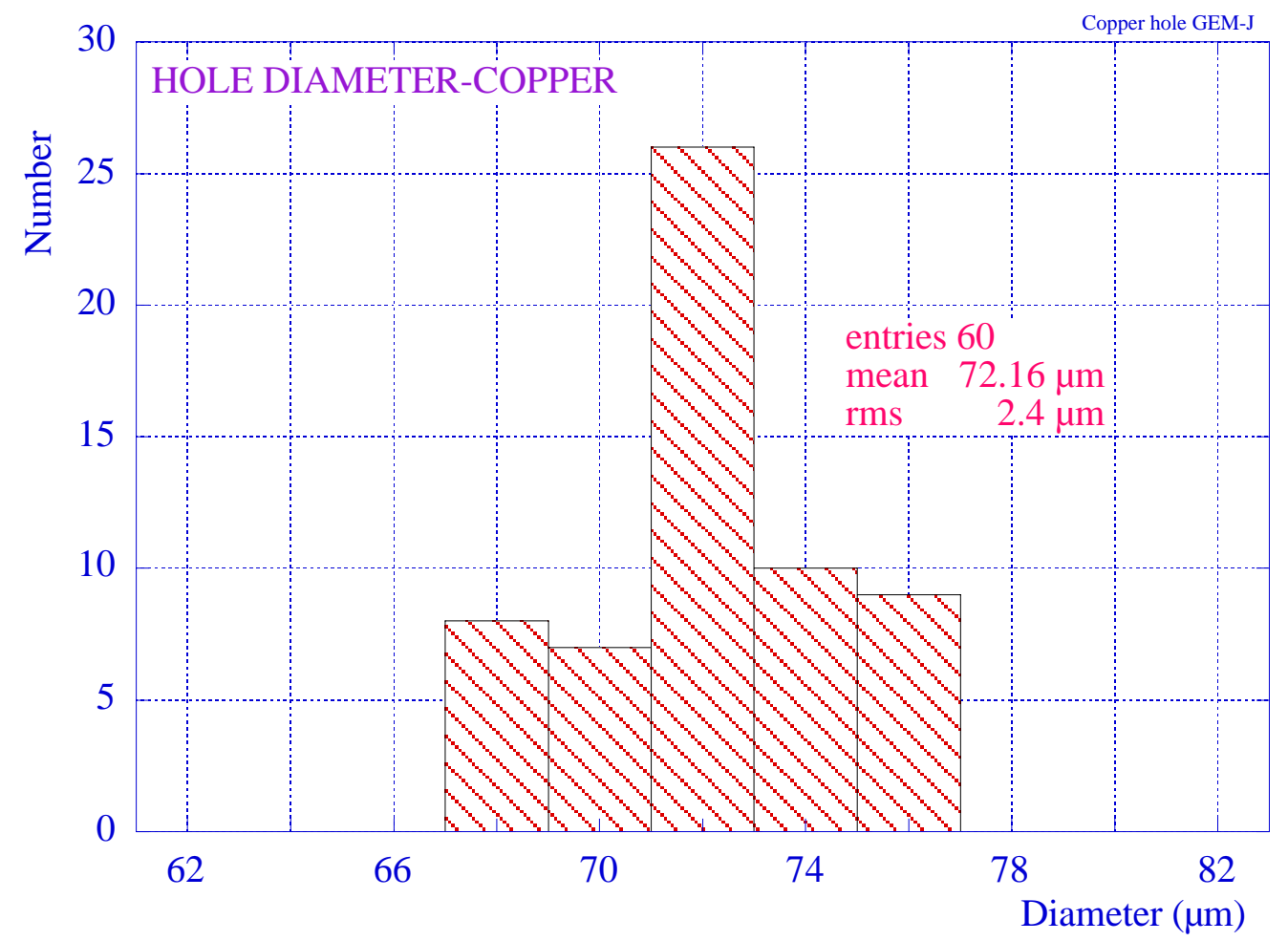

Fig. 8: Measured distribution of the hole diameters at the metal level.

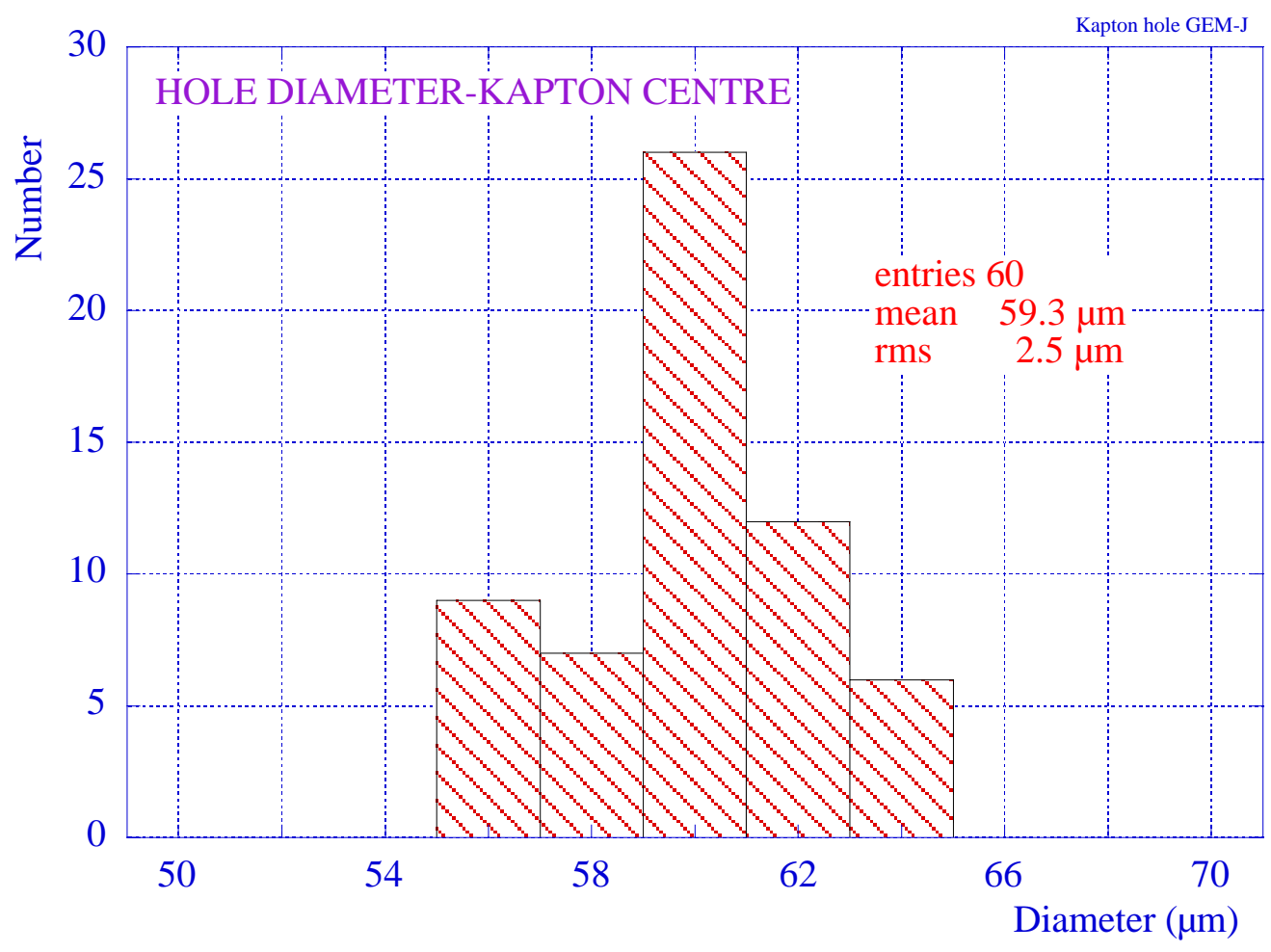

Fig. 9: Measured distribution of the hole diameters at the kapton center. 


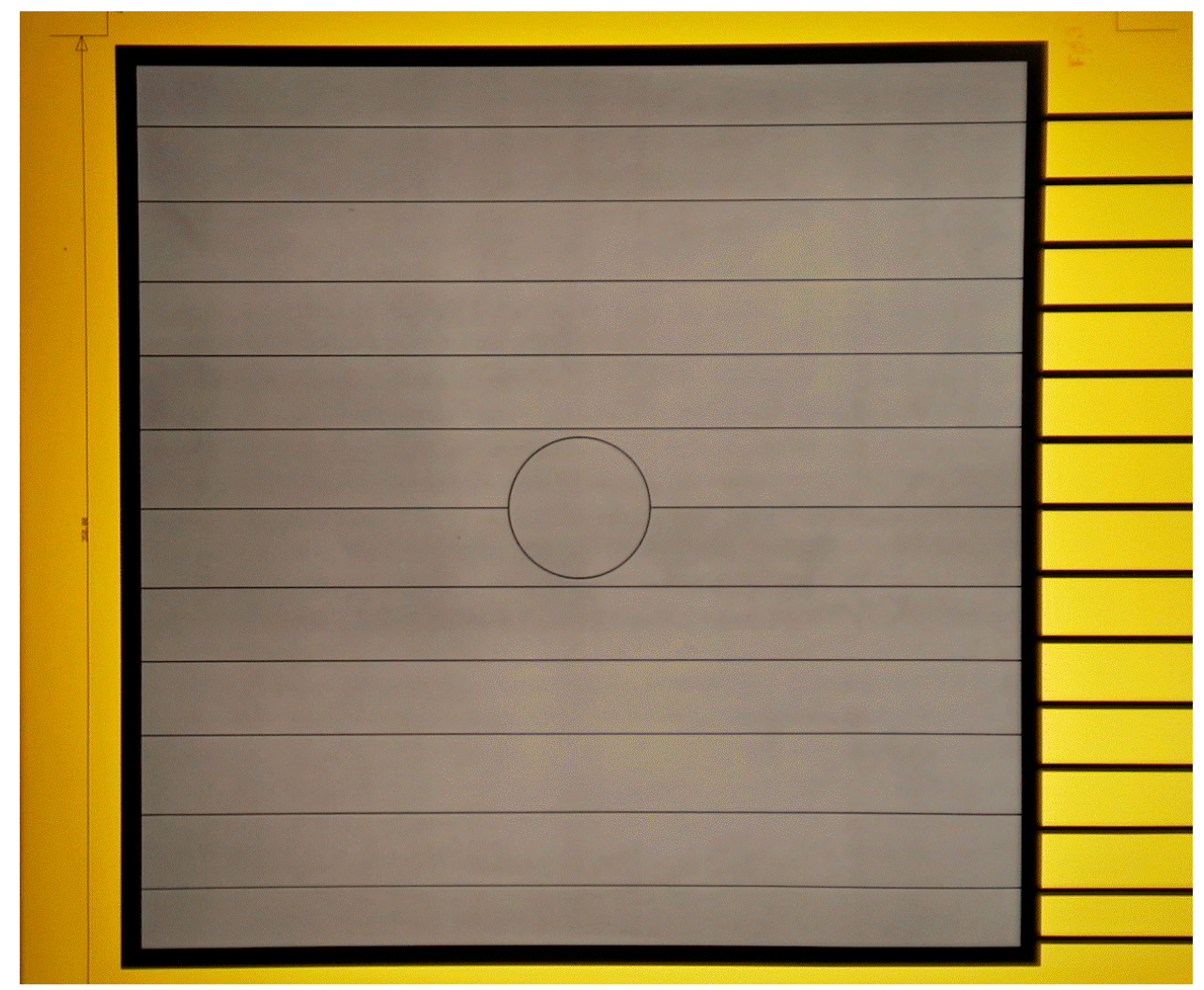

Fig. 10: A GEM electrode seen from the partitioned side

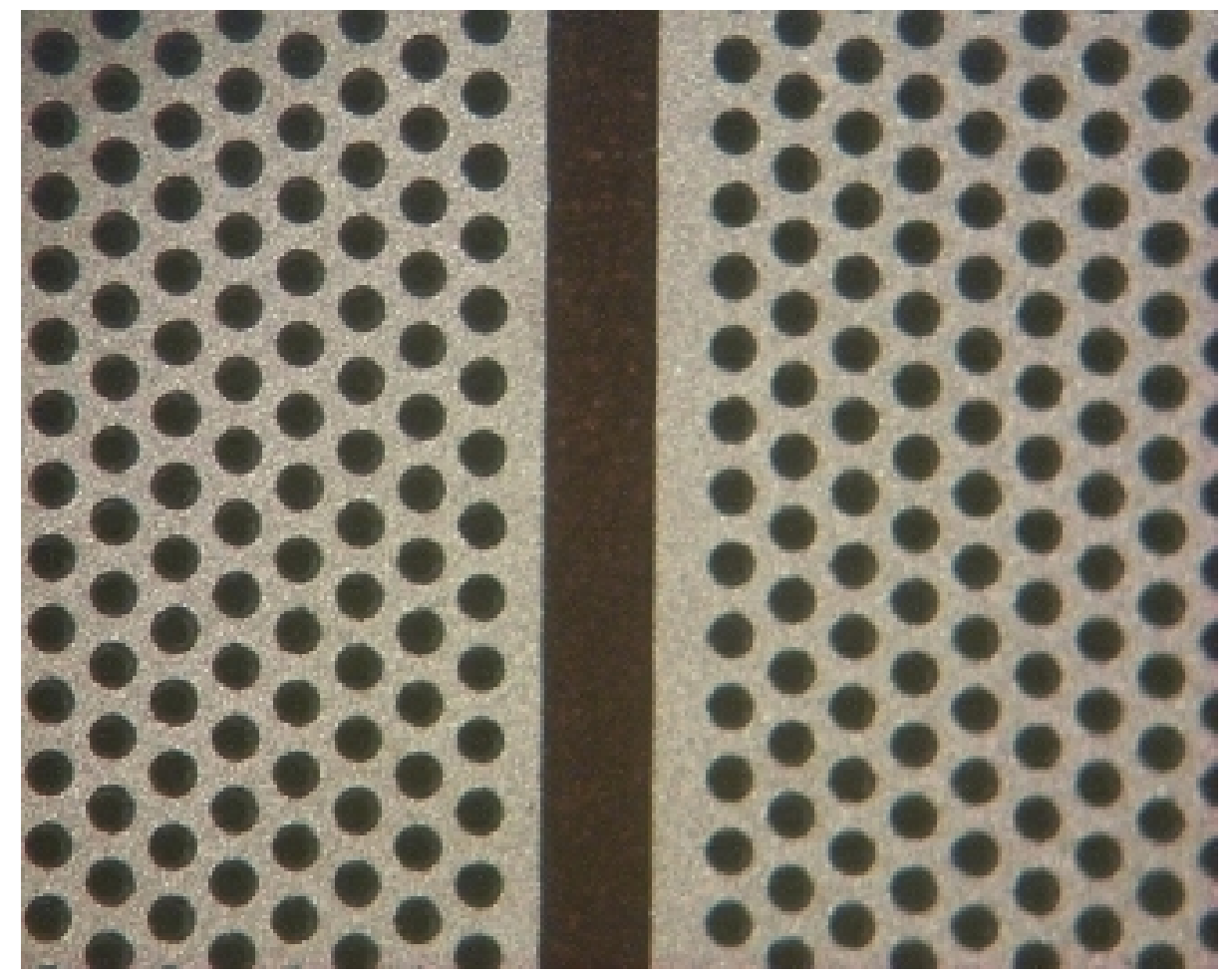

Fig. 11: Close view of a sector's separation, $200 \mu \mathrm{m}$ wide. 


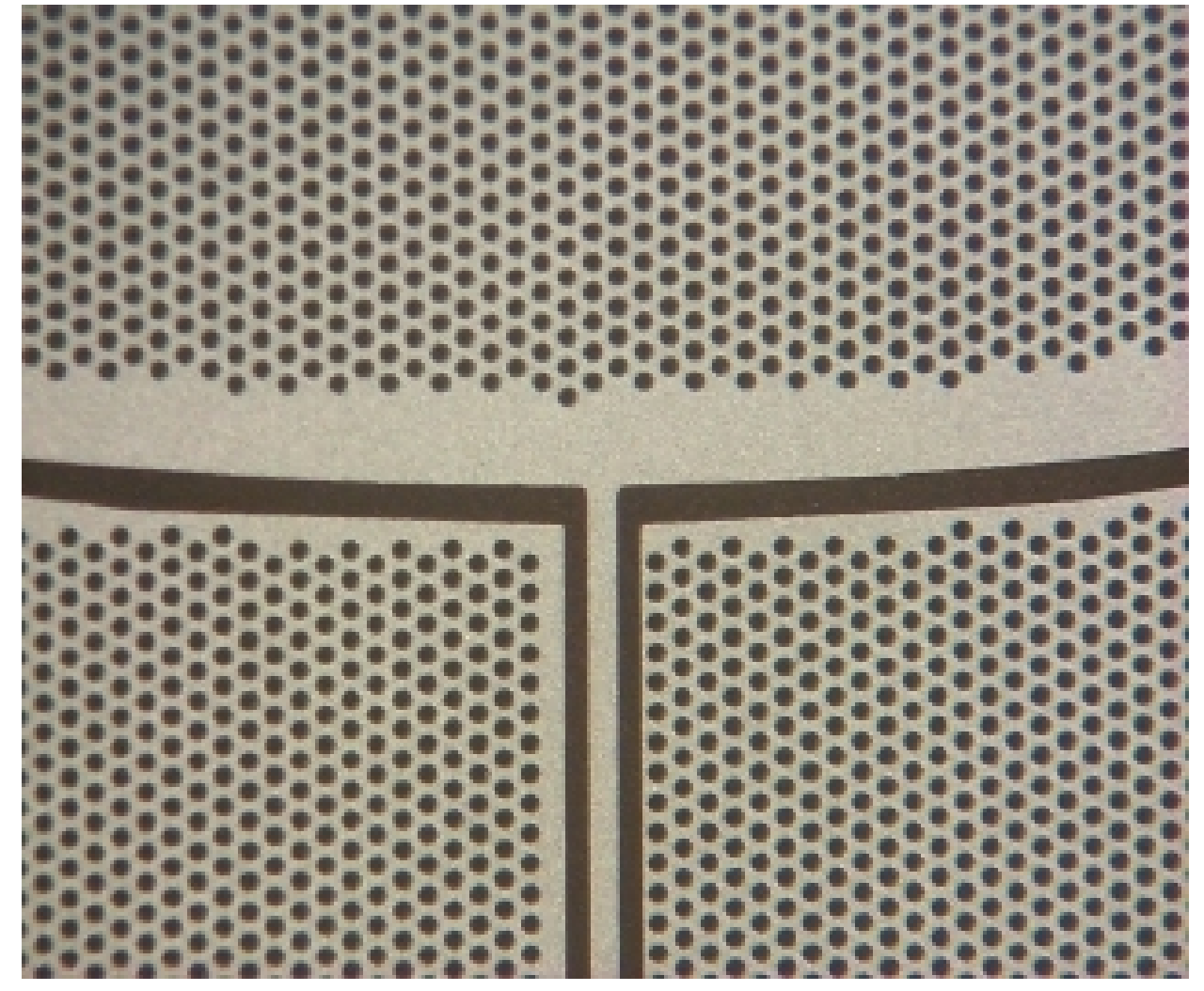

Fig. 12: Close-up of the region of electrical contact to the central beam killer.

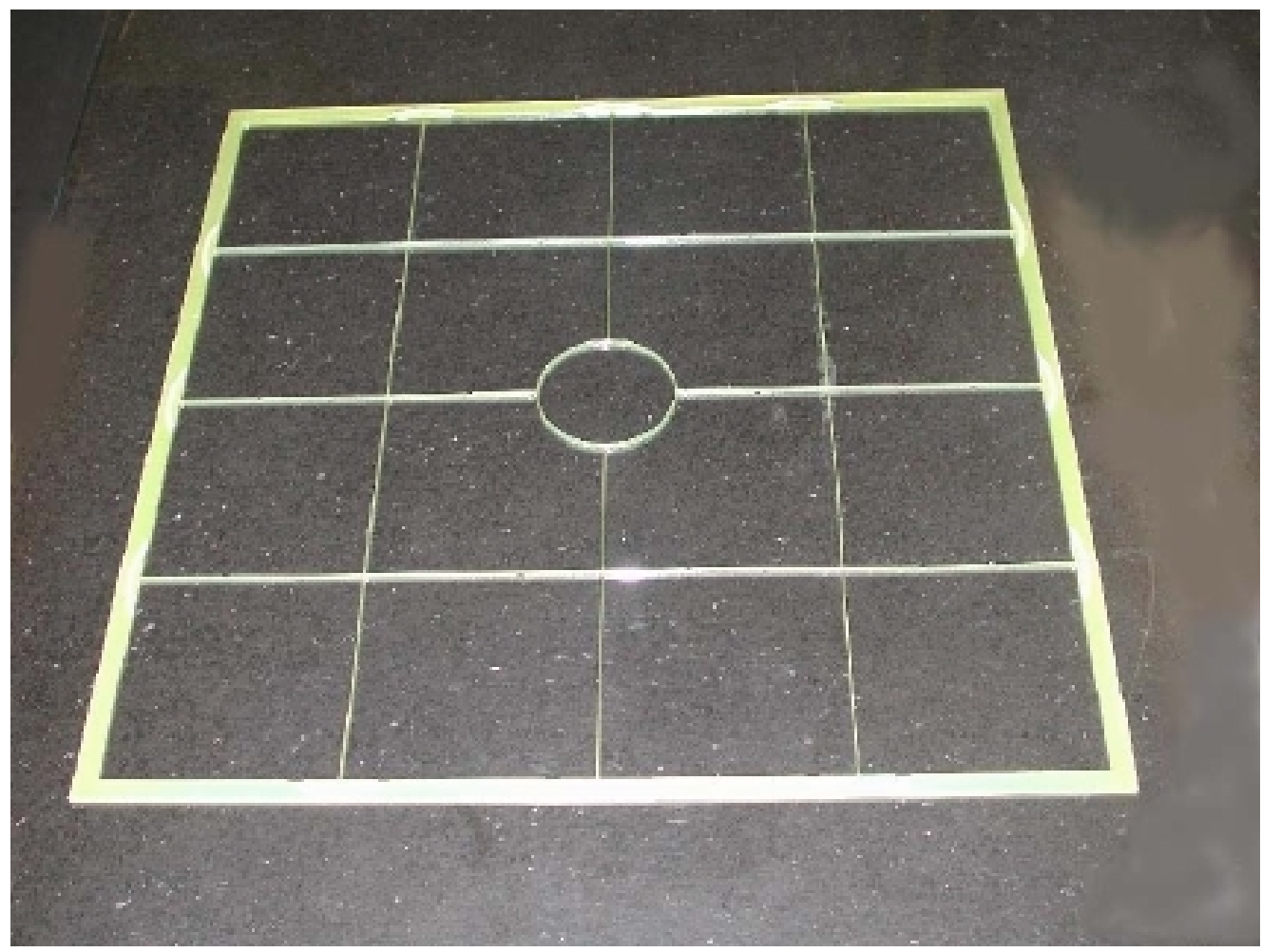

Fig. 13: GEM assembly frame with spacer grid. 


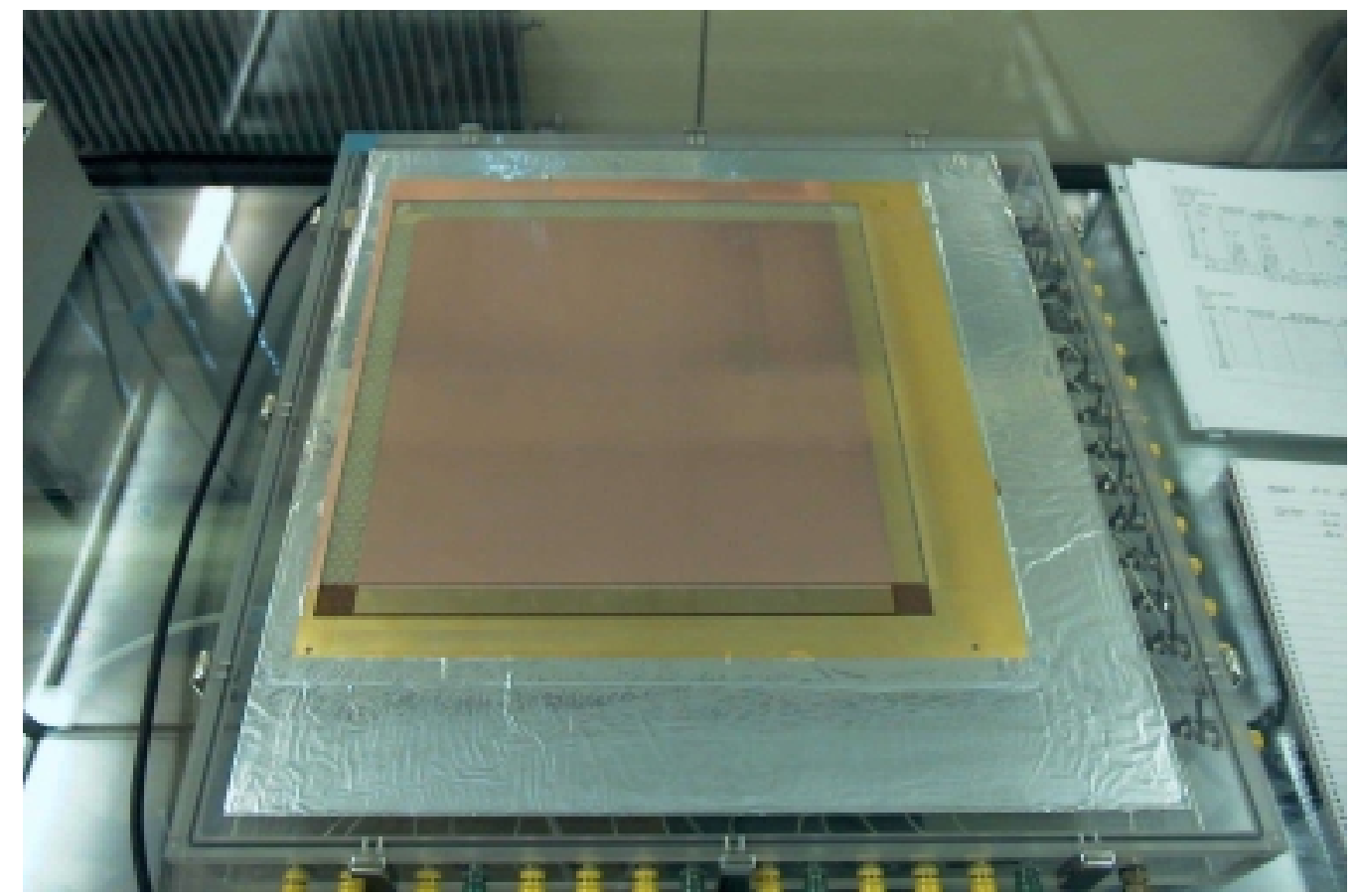

Fig. 14: A 2-dimensional projective readout board, glued to the large honeycomb supporting plate.

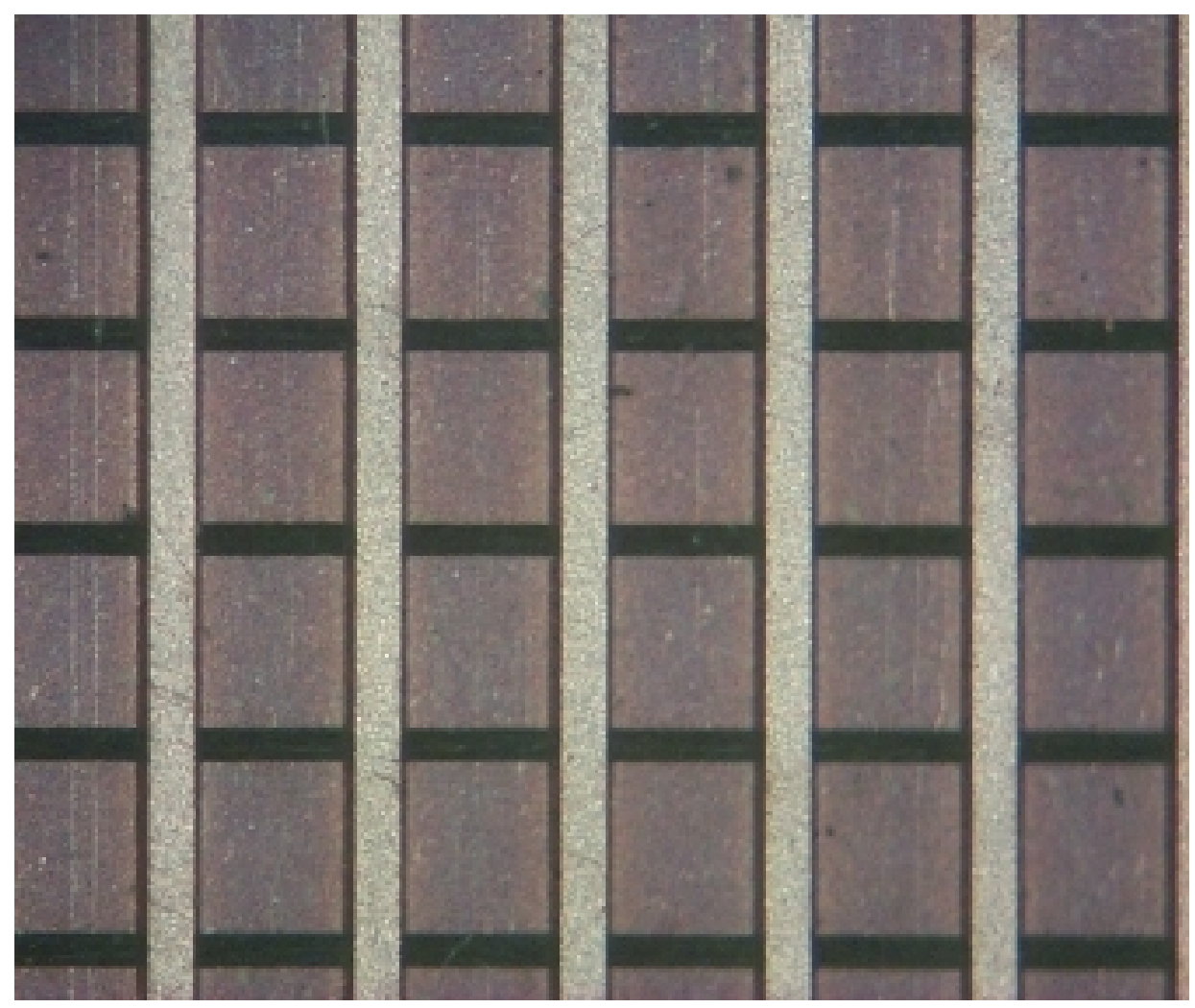

Fig. 15: Close view of the readout board; strip are 80 and $340 \mu \mathrm{m}$ wide, respectively, at $400 \mu \mathrm{m}$ pitch and separated by $50 \mu \mathrm{m}$ kapton ridges. 


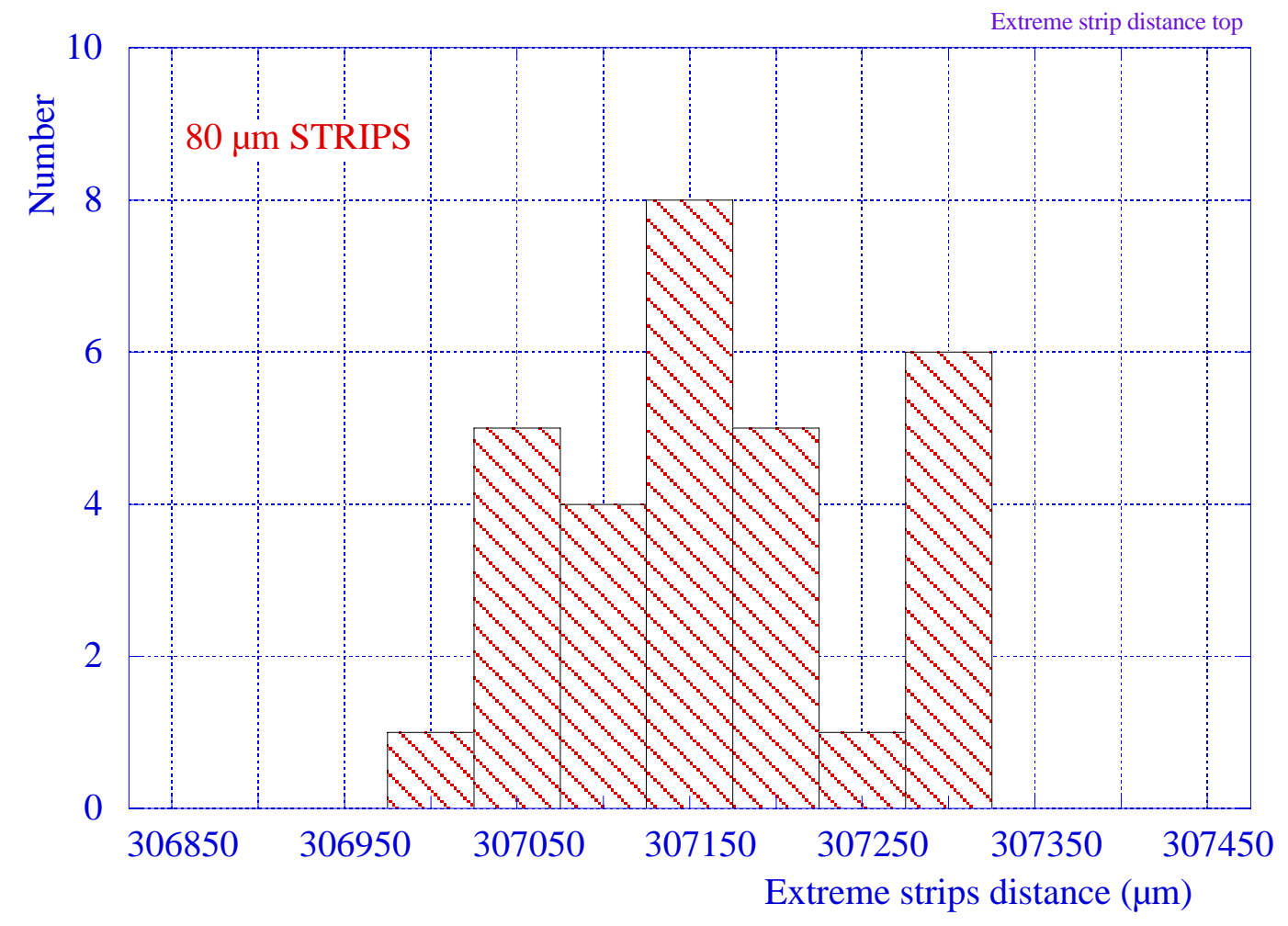

(a)

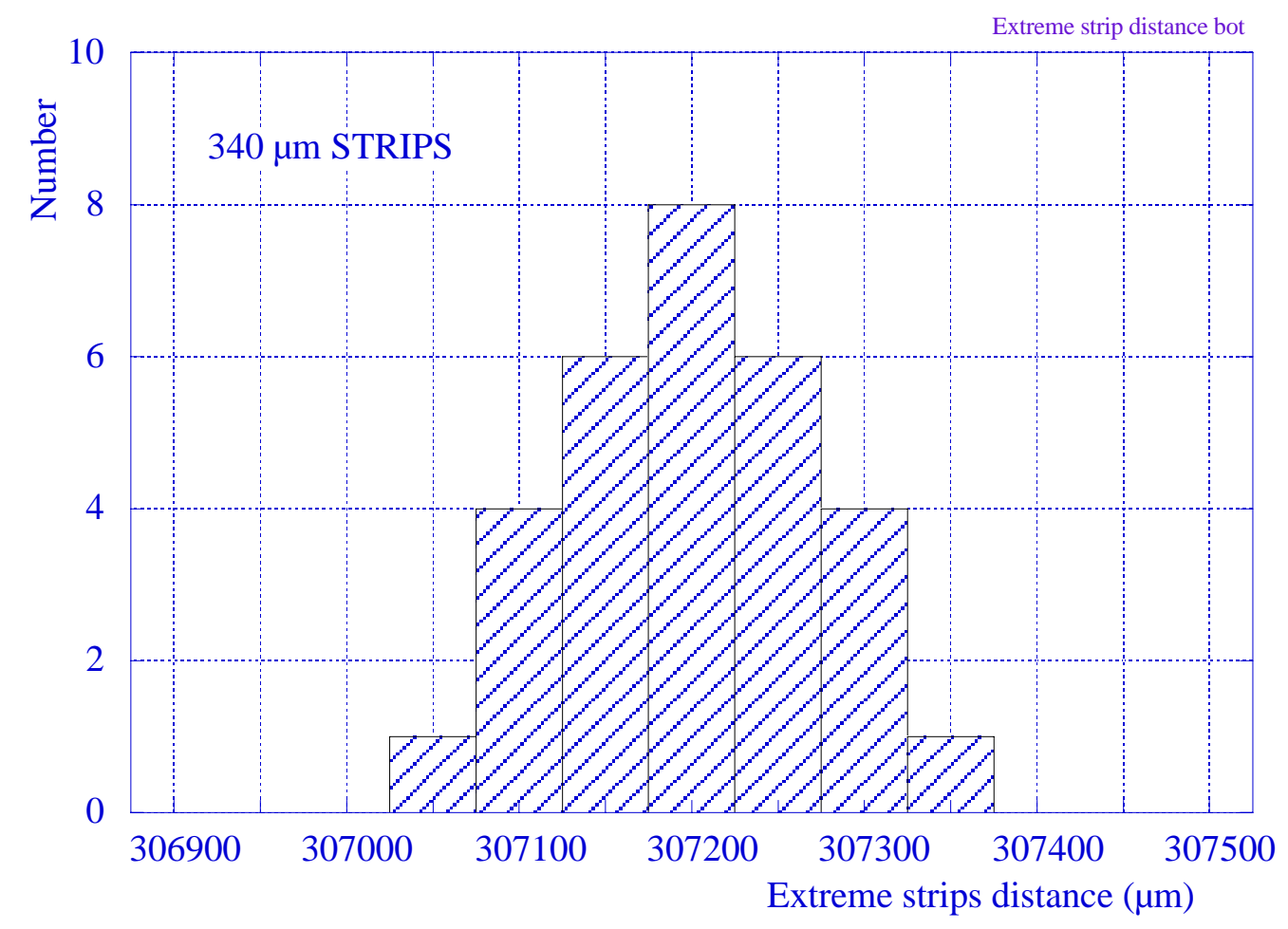

(b)

Fig. 16: Measured distributions of distance between extreme strips on a readout board, for the narrow (a) and wide strips (b). 


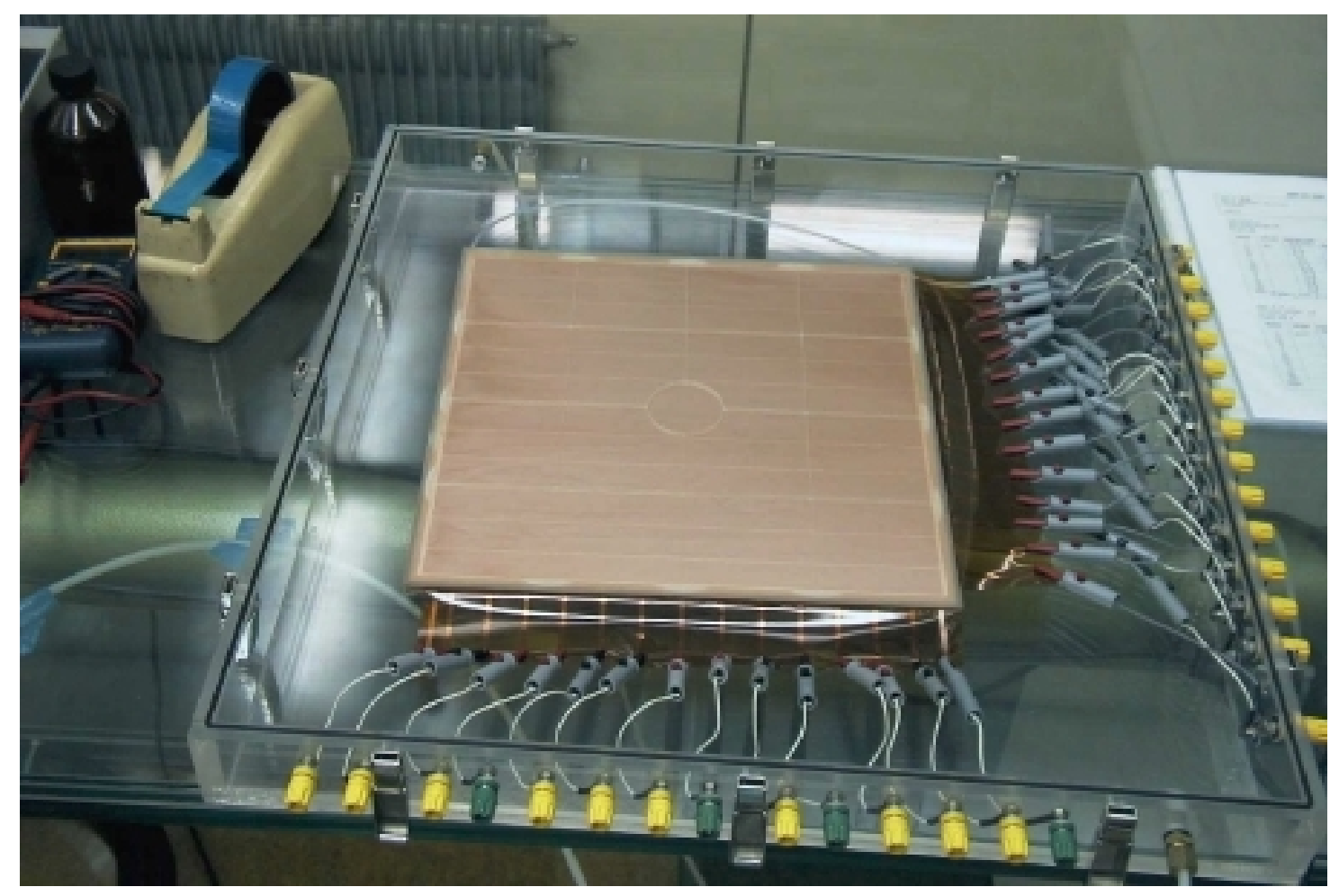

Fig. 17: The nitrogen-filled box used for systematic HV testing.

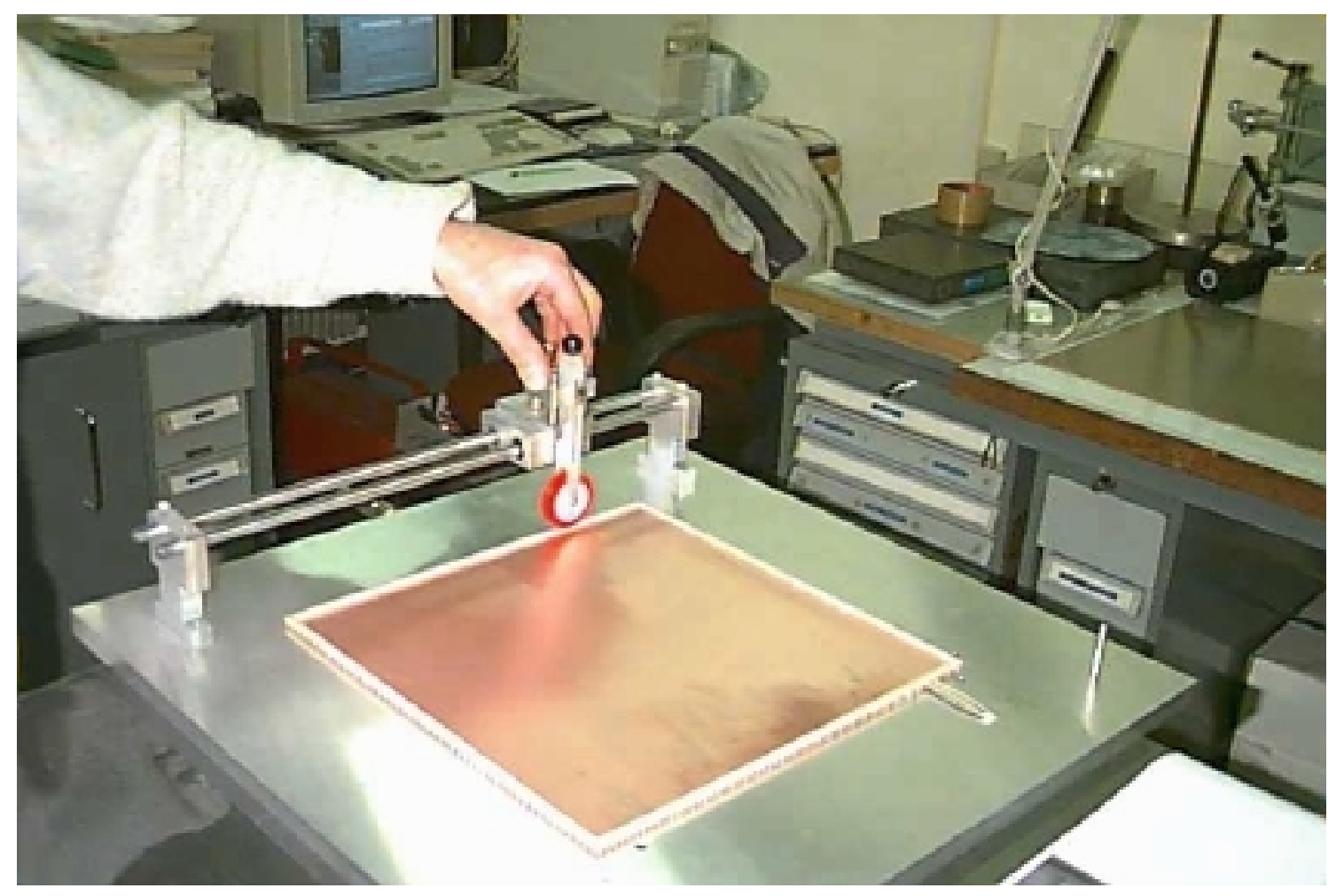

Fig. 18: View of the assembly table and epoxy-coating tool. 


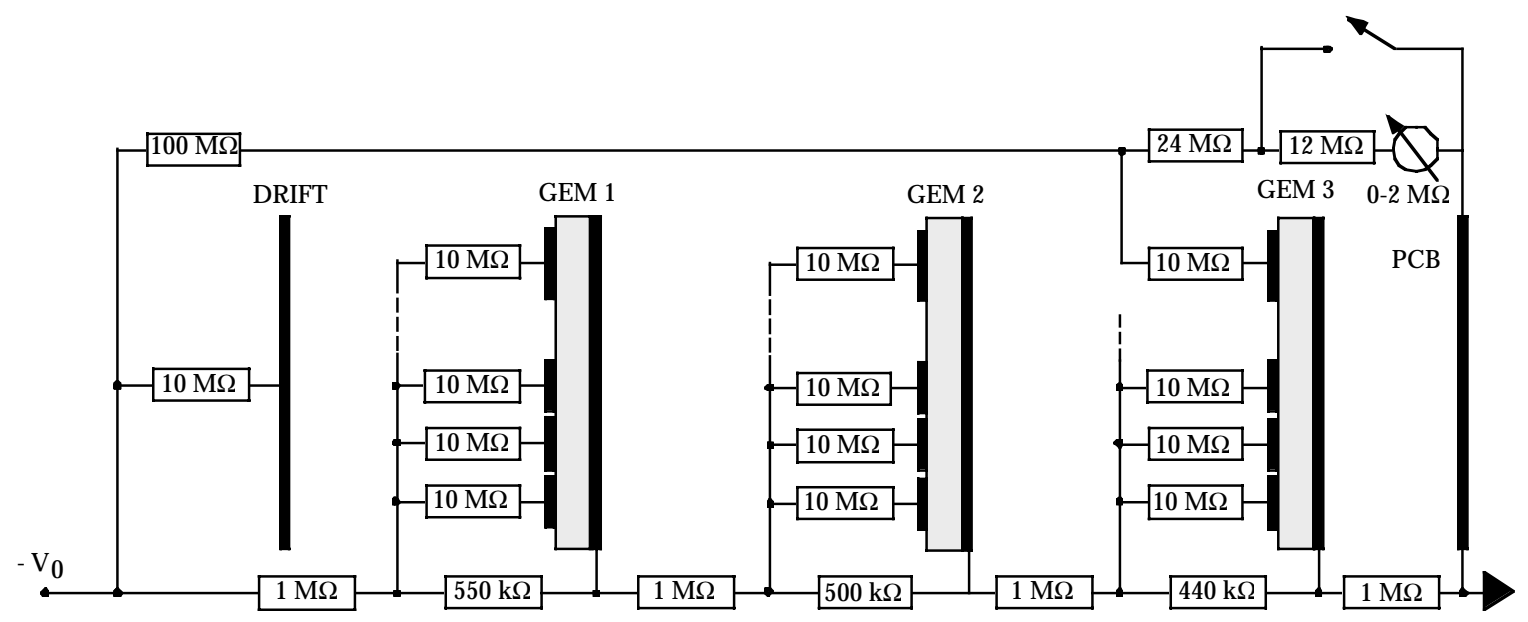

Fig. 19: The resistor chain used for high voltage distribution. A remotecontrolled switch allows to activate/de-activate the central beam region.

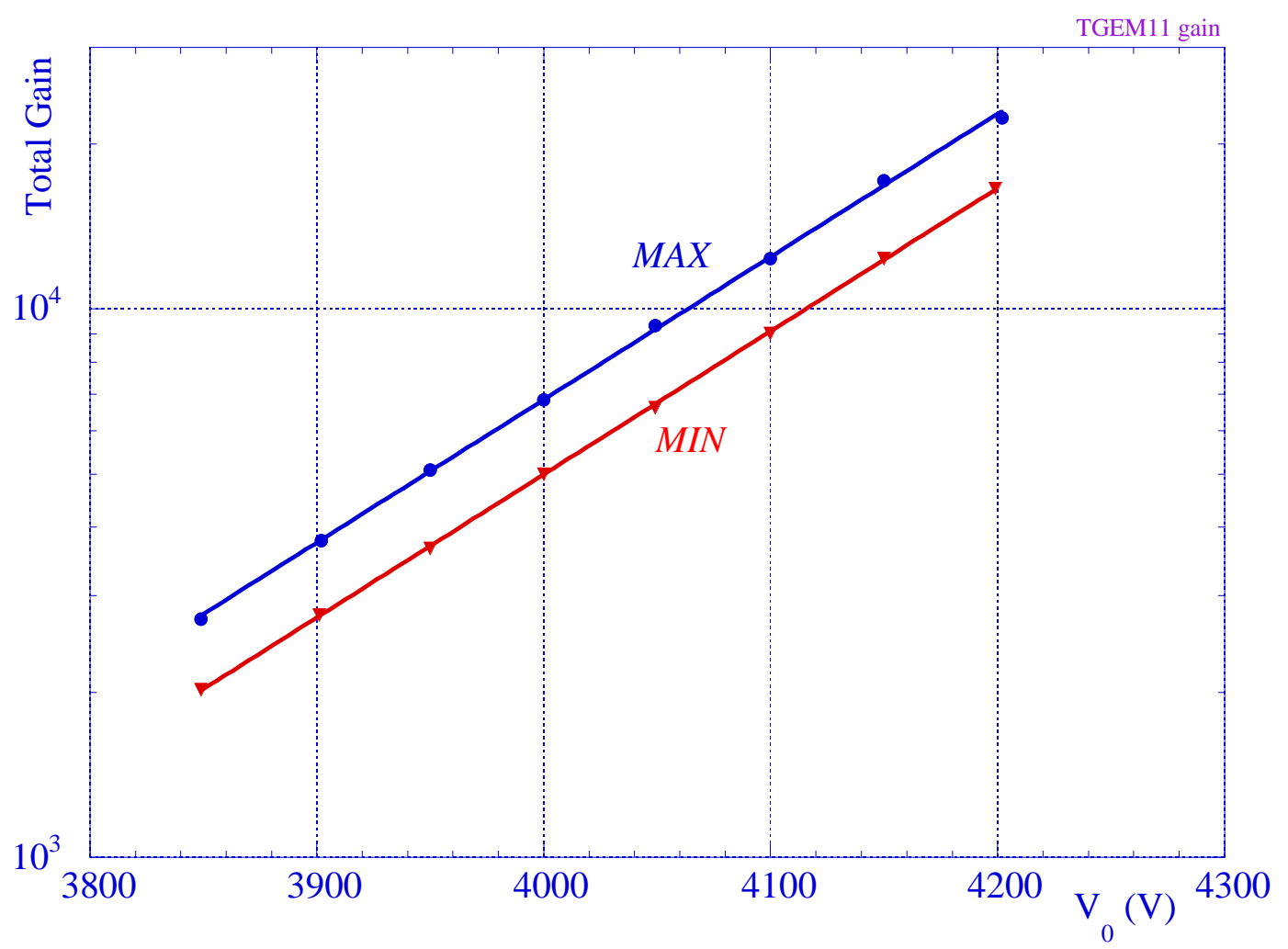

Fig. 20: Absolute effective gain measured in two positions of a chamber with extreme values. 


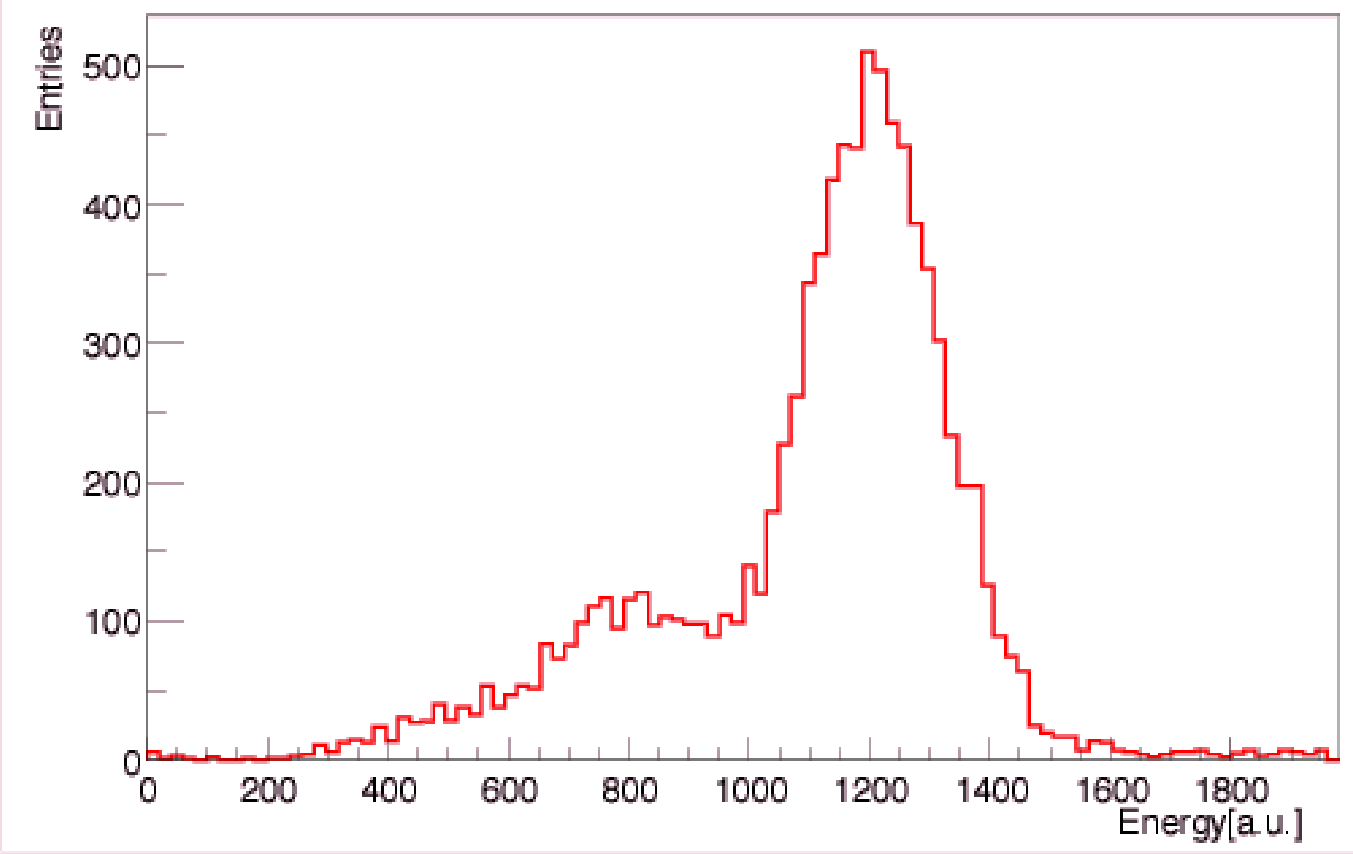

(a)

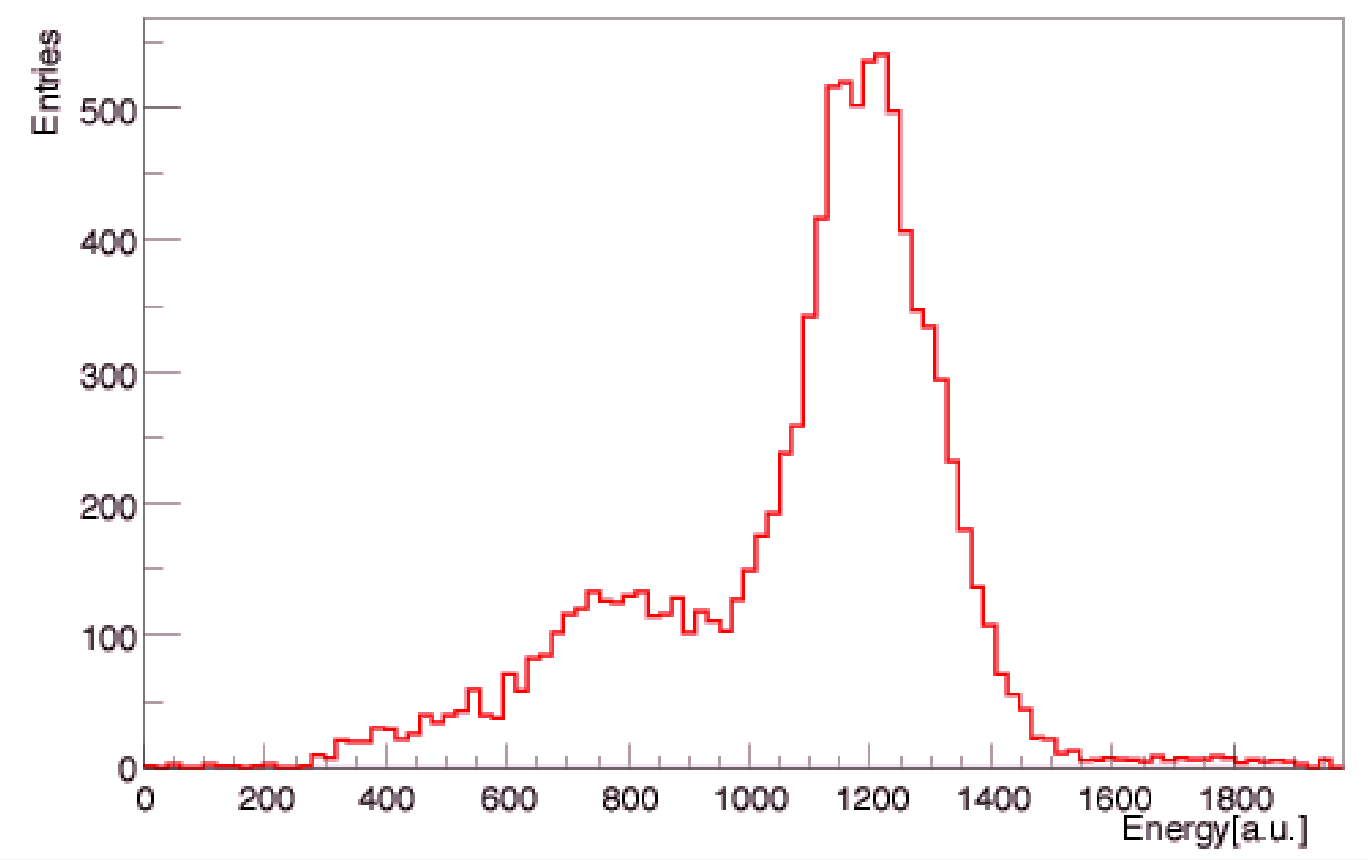

(b)

Fig. 21: Typical pulse height spectra for $9 \mathrm{keV} \mathrm{X}$-rays recorded on groups of strips in the $x(a)$ and $y(b)$ projections. 


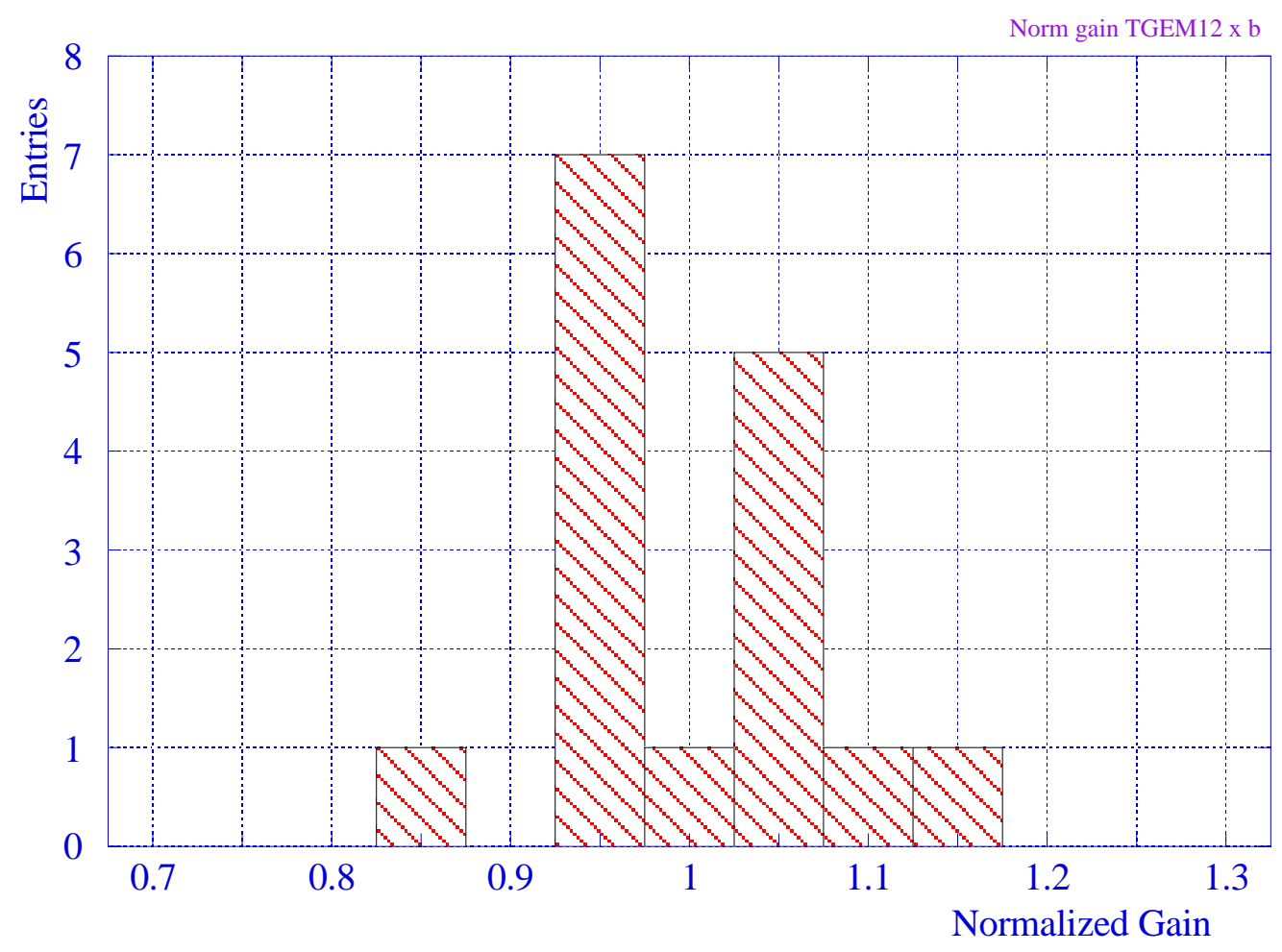

(a)

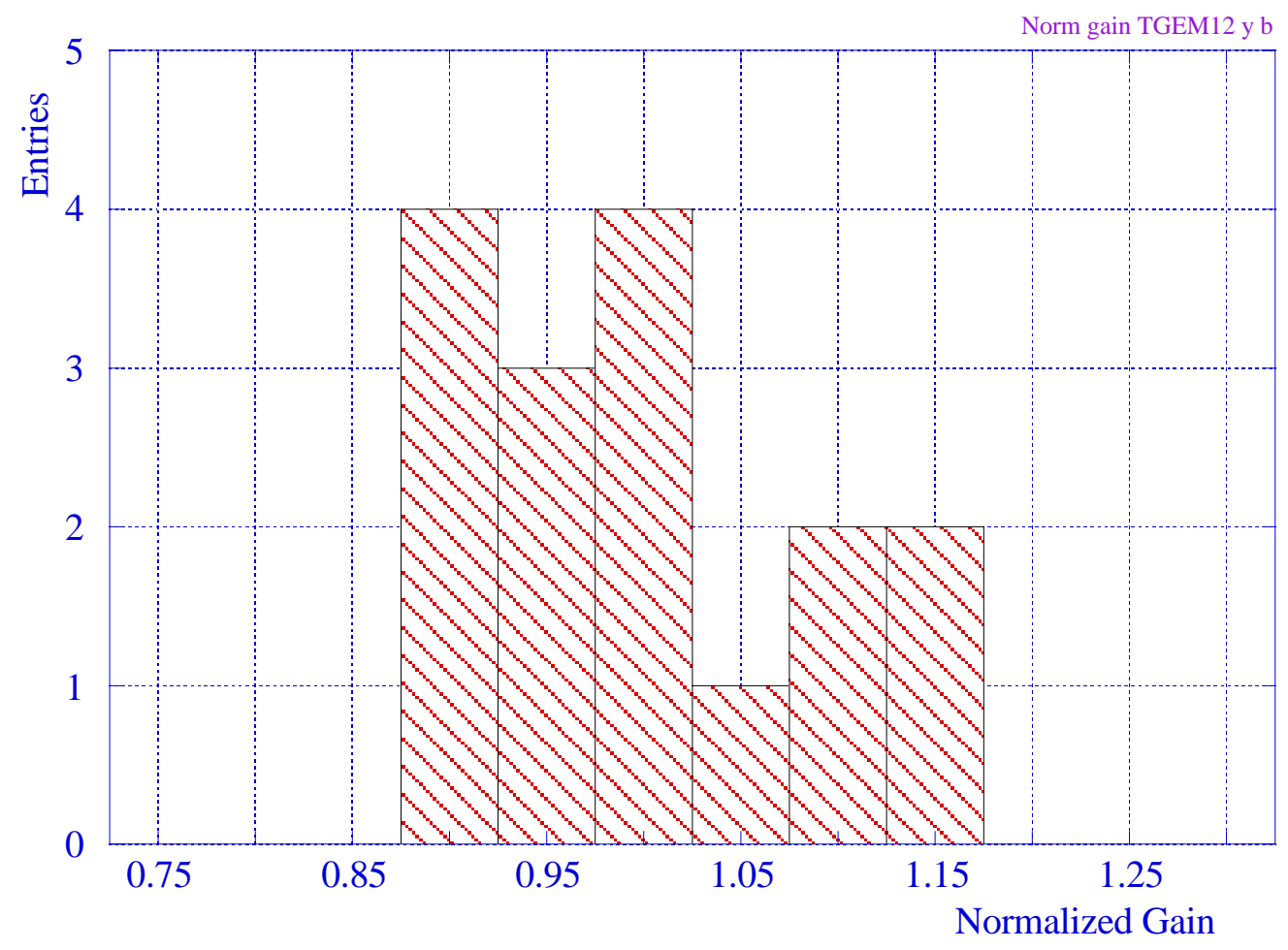

(b)

Fig. 22: Distribution of normalized gains measured on 16 points in the detector along the $\mathrm{x}(\mathrm{a})$ and $\mathrm{y}(\mathrm{b})$ projections. 


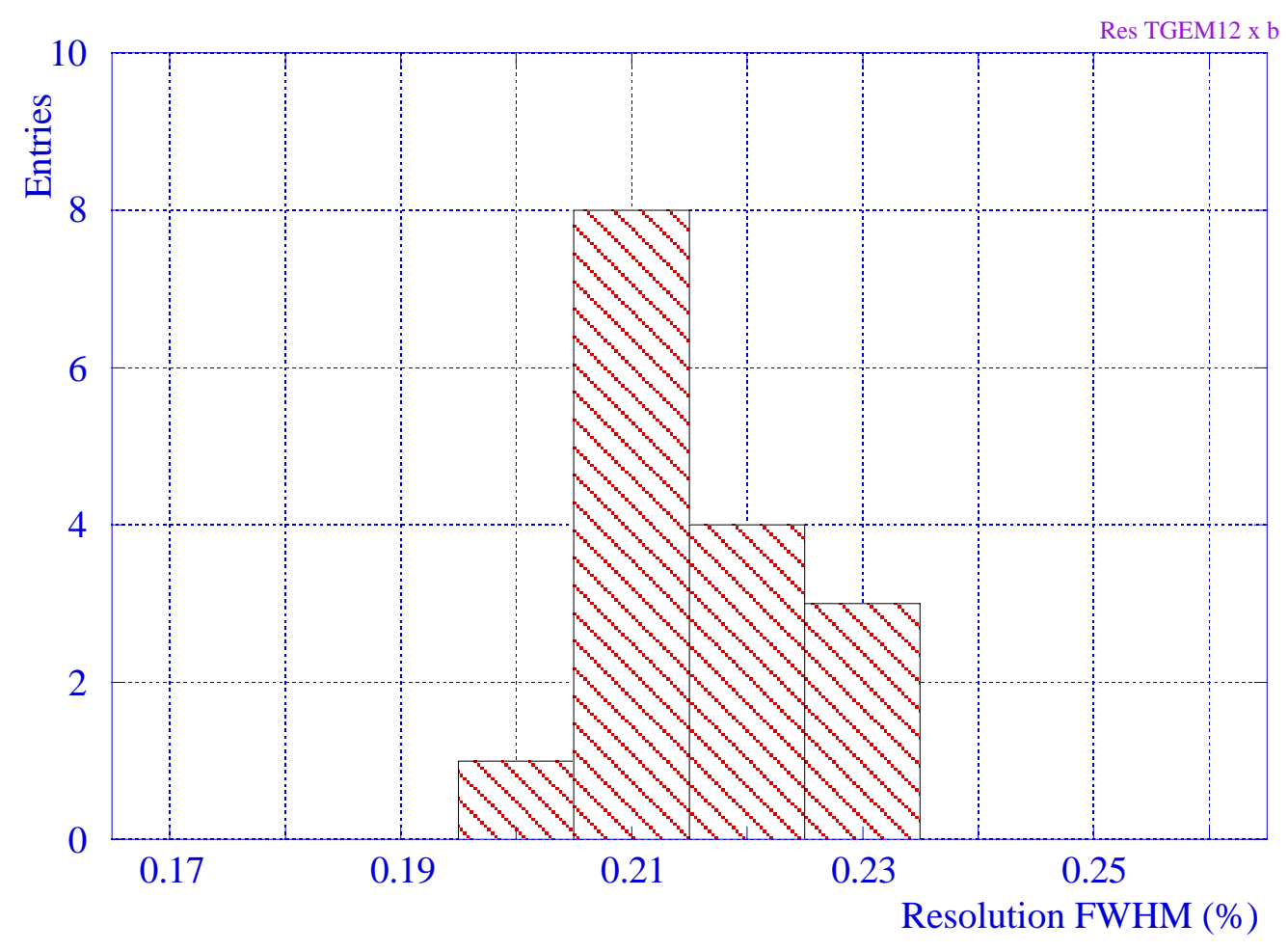

(a)

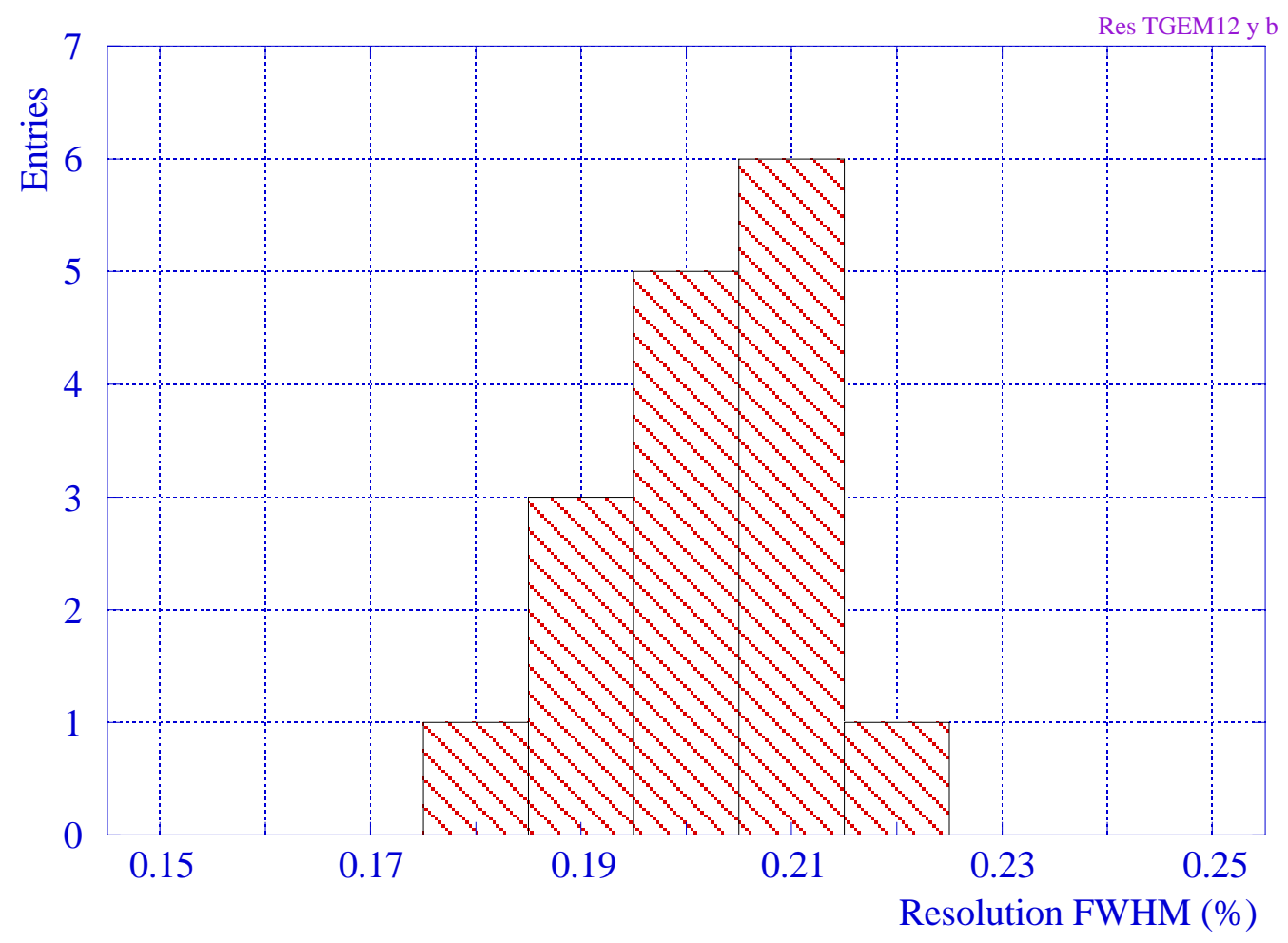

(b)

Fig. 23: Distribution of energy resolution measured on 16 points in the same detector along the $\mathrm{x}(\mathrm{a})$ and $\mathrm{y}(\mathrm{b})$ projections. 


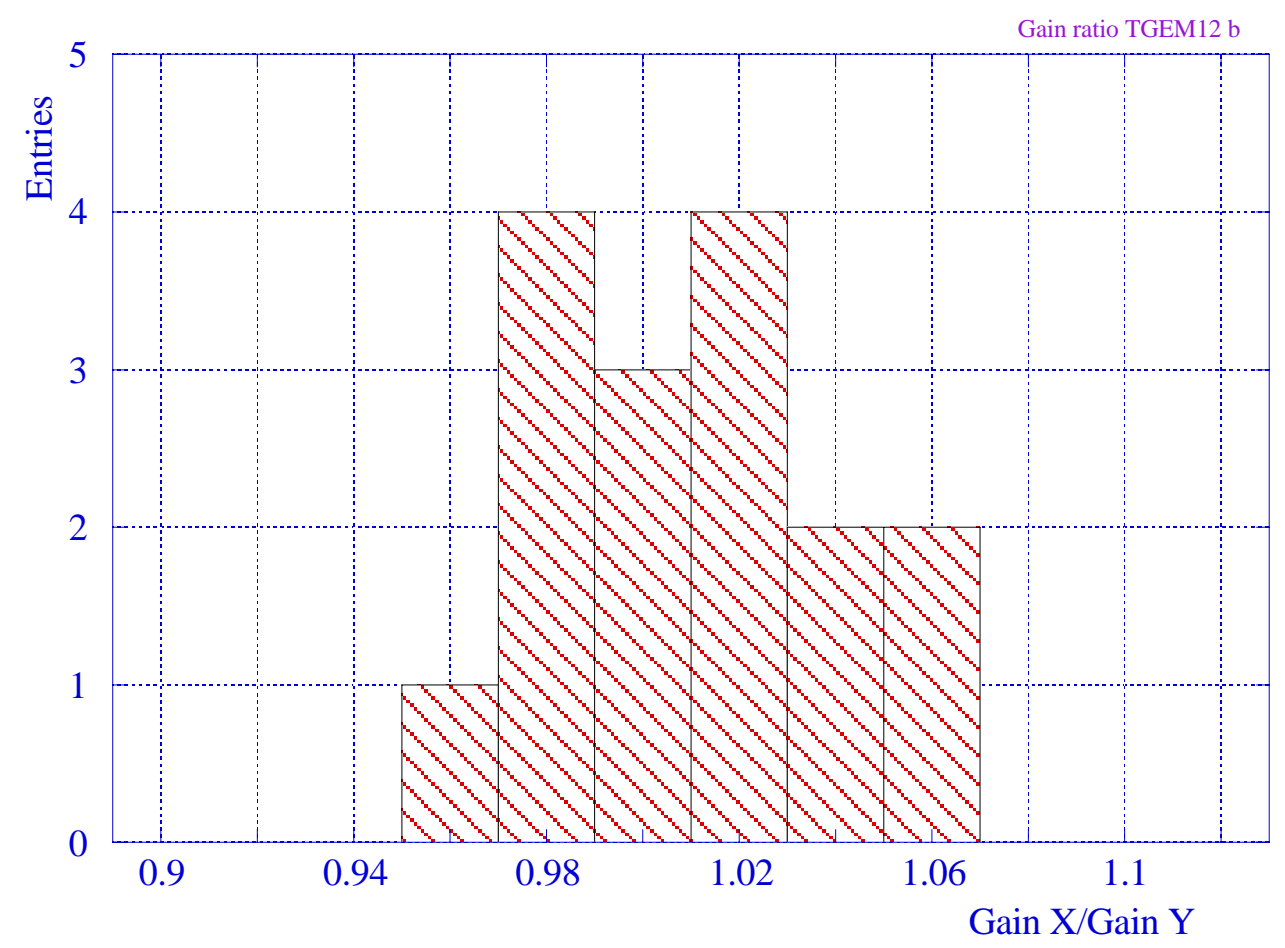

Fig. 24: Ratio of gains measured on the $x$ and $y$ coordinates.

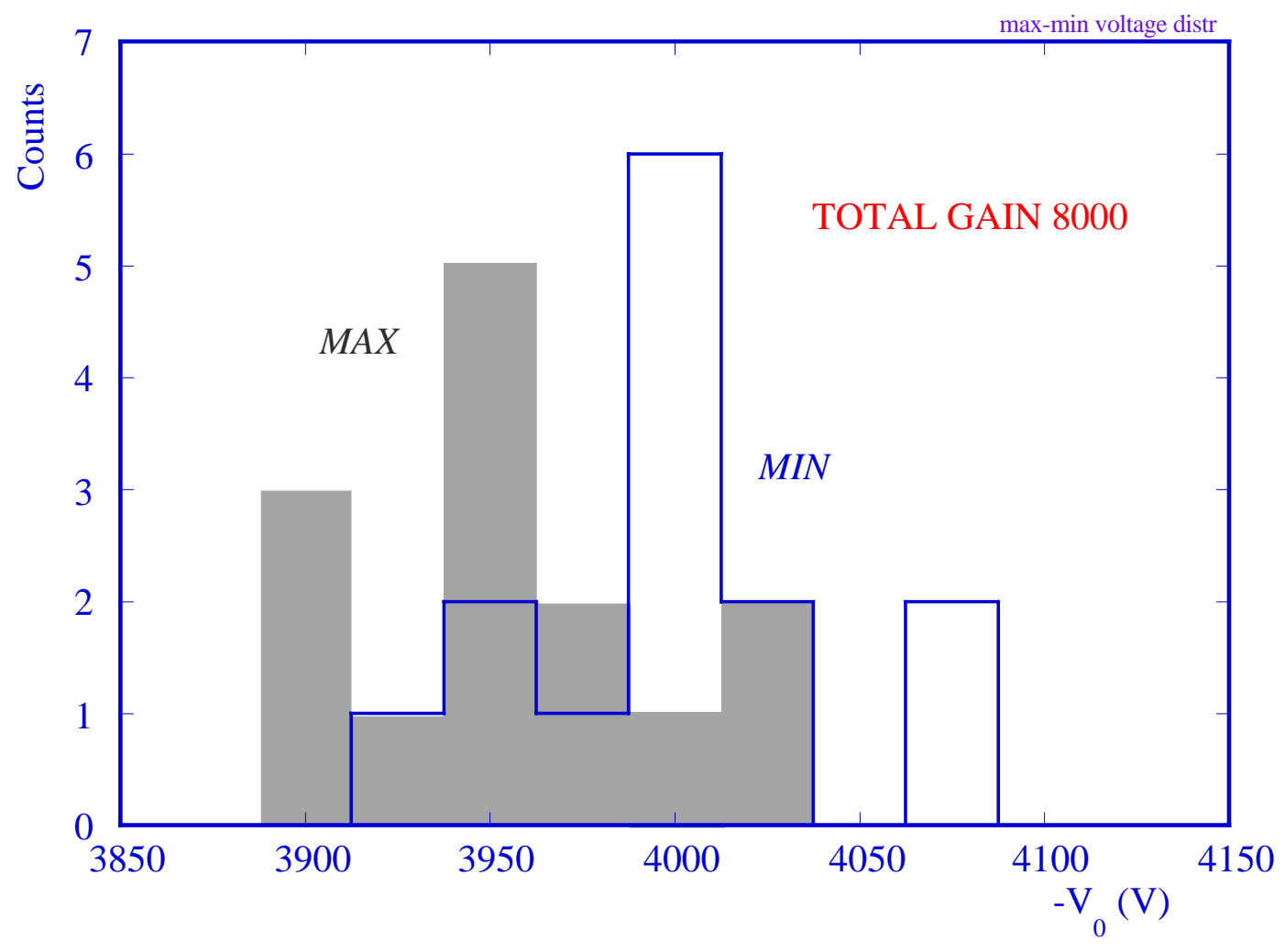

Fig. 25: Distribution of the voltages required for an effective gain of 8000 in the minimum and maximum gain region of 8 detectors. Both geometry and variations in the resistive voltage dividers contribute to the spread. 


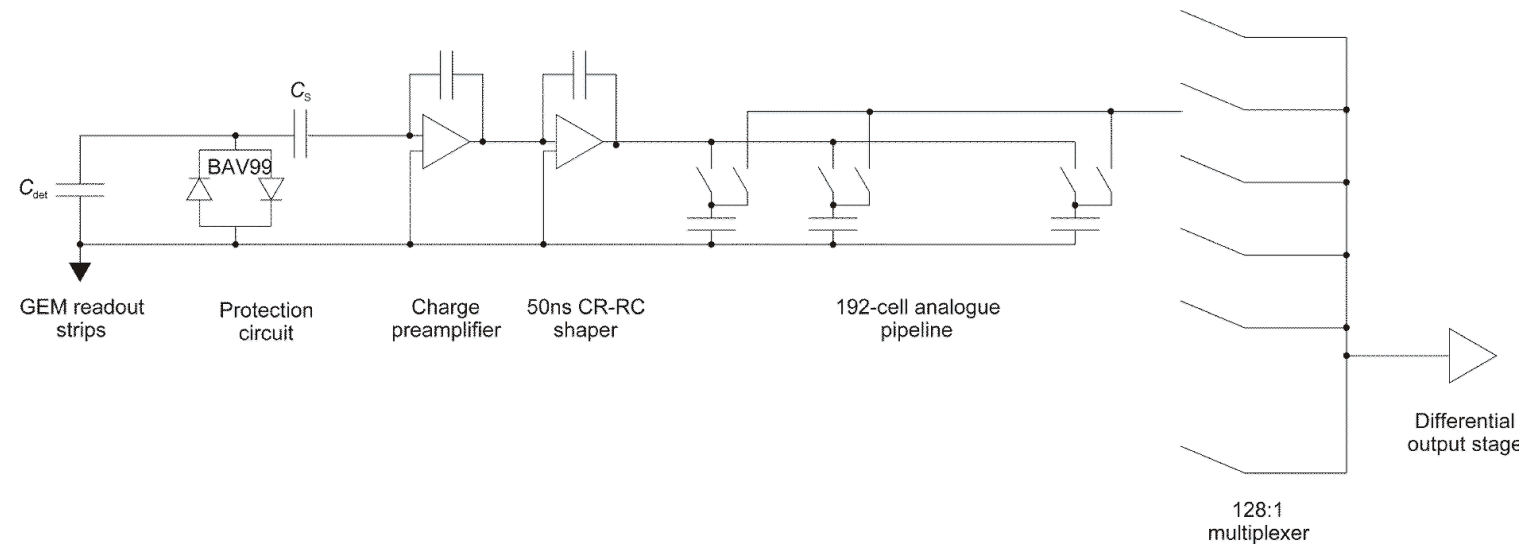

Fig. 26: Schematics of the channel read-out electronics, with the diode protection and APV25 analogue pipeline.

FE boards

(3 APV each)

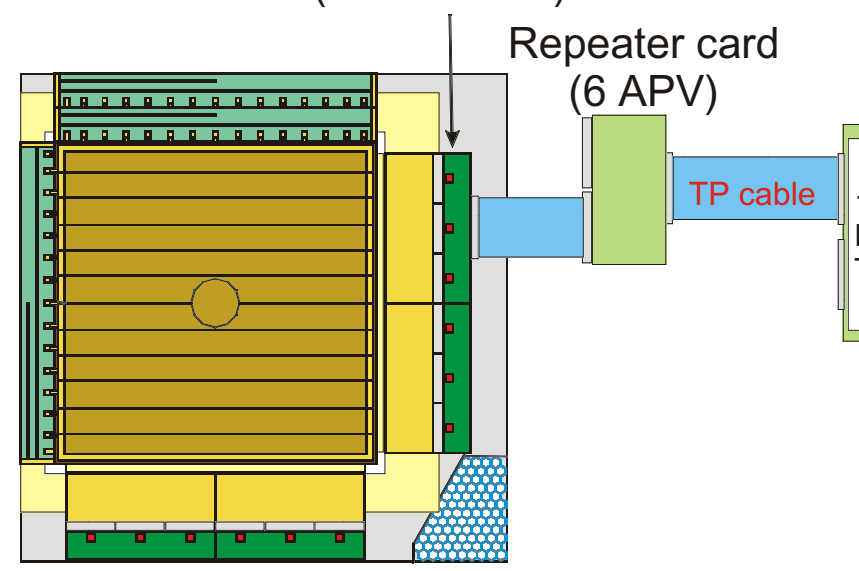

ADC (12 APV)

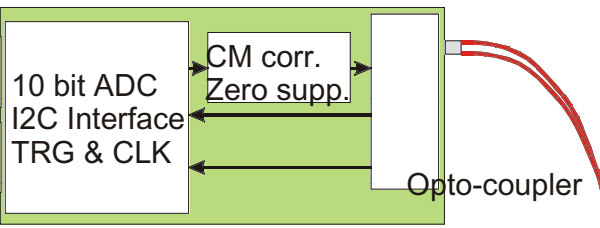

Optical fibre

Hotlink

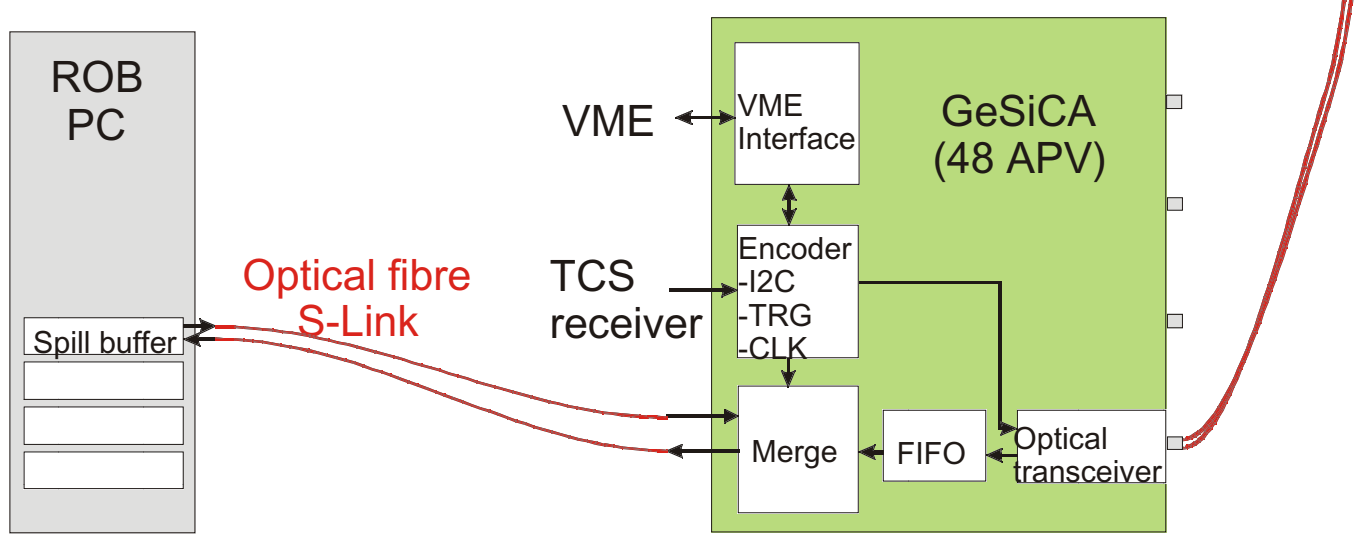

Fig. 27: Schematic block diagram of the readout electronics. 


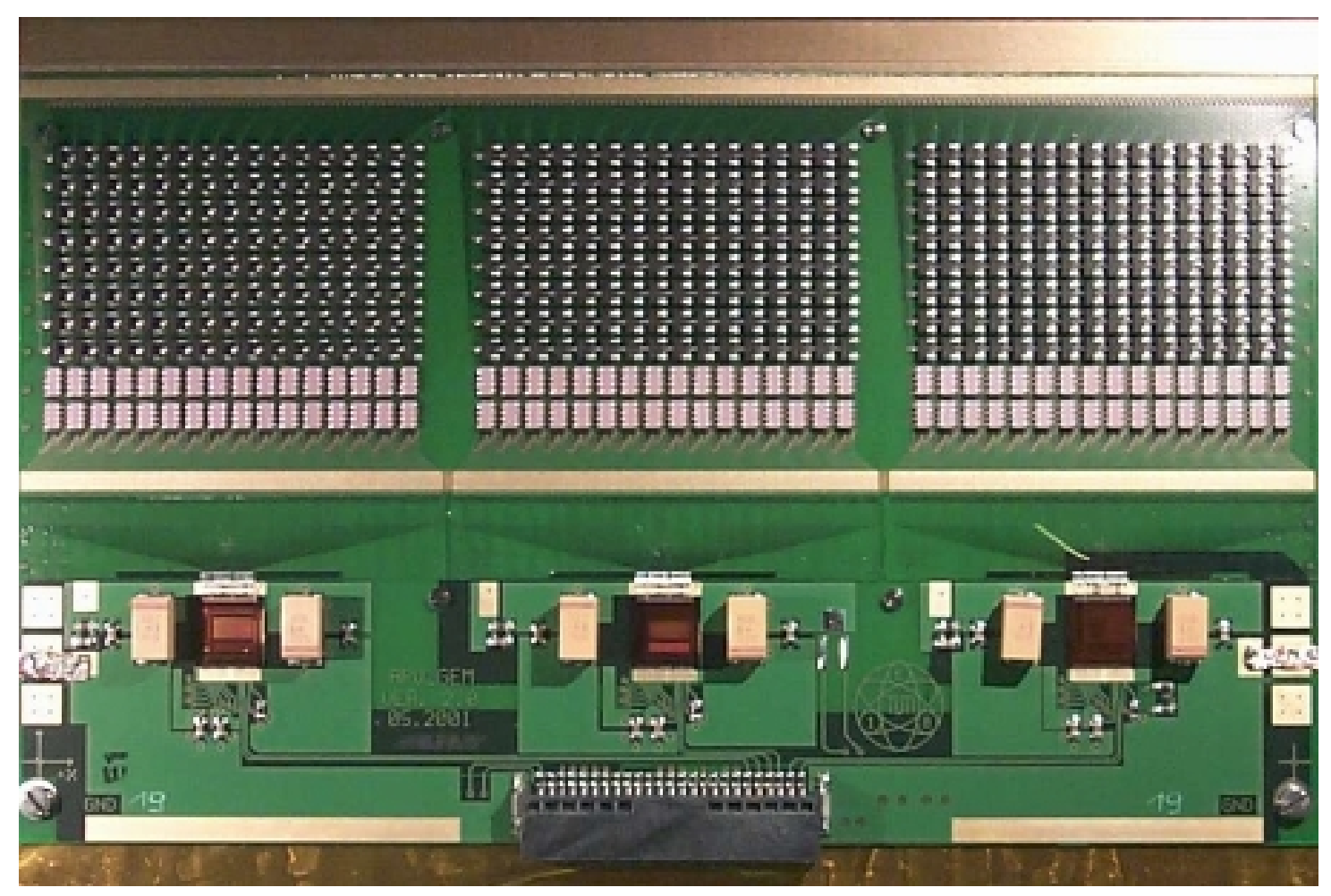

Fig. 28: Close-up of a read-out card, mounting three APV25 chips and the input protections for 384 channels.

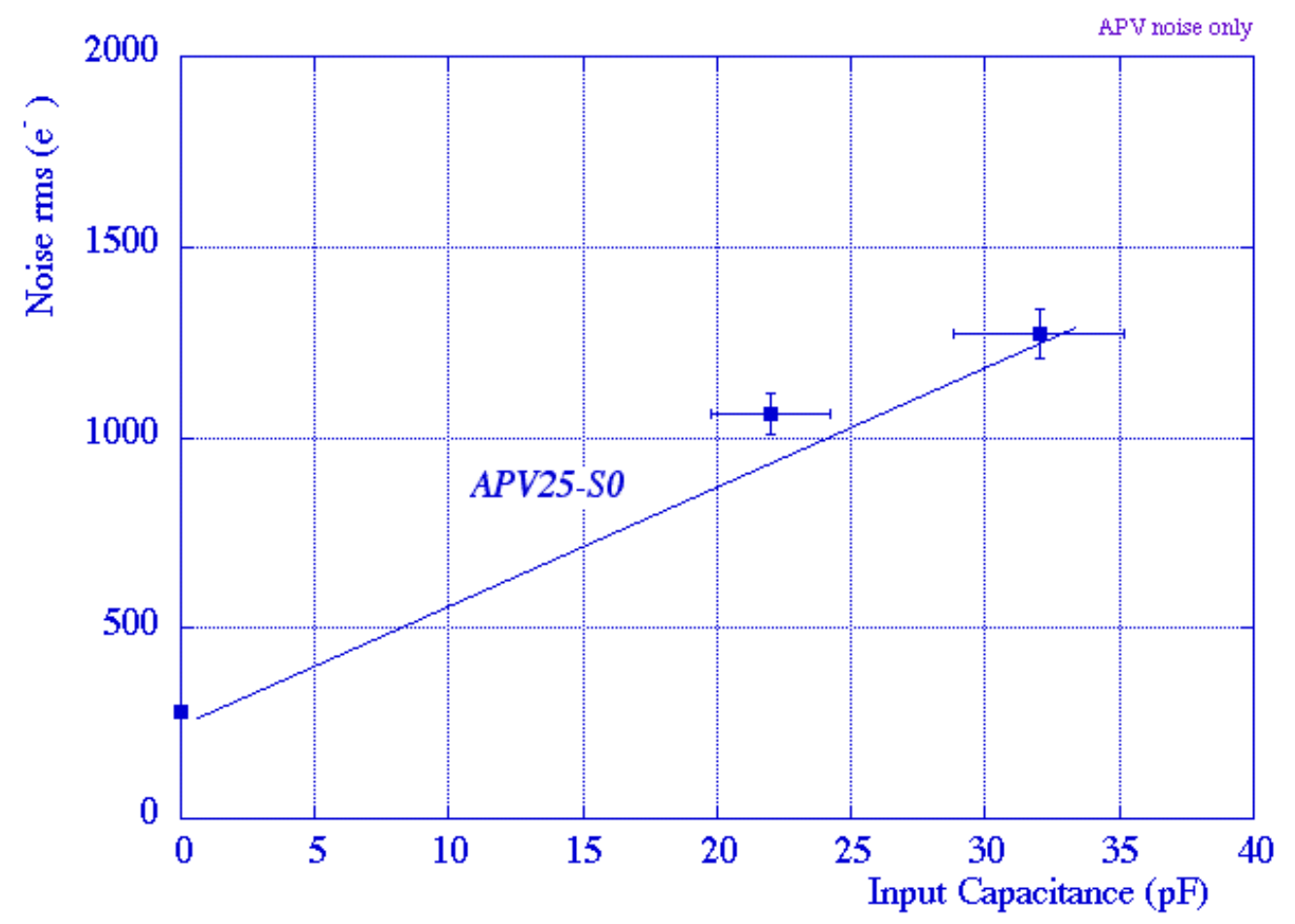

Fig. 29: Computed (full line) and measured input noise as a function of strip capacitance. 


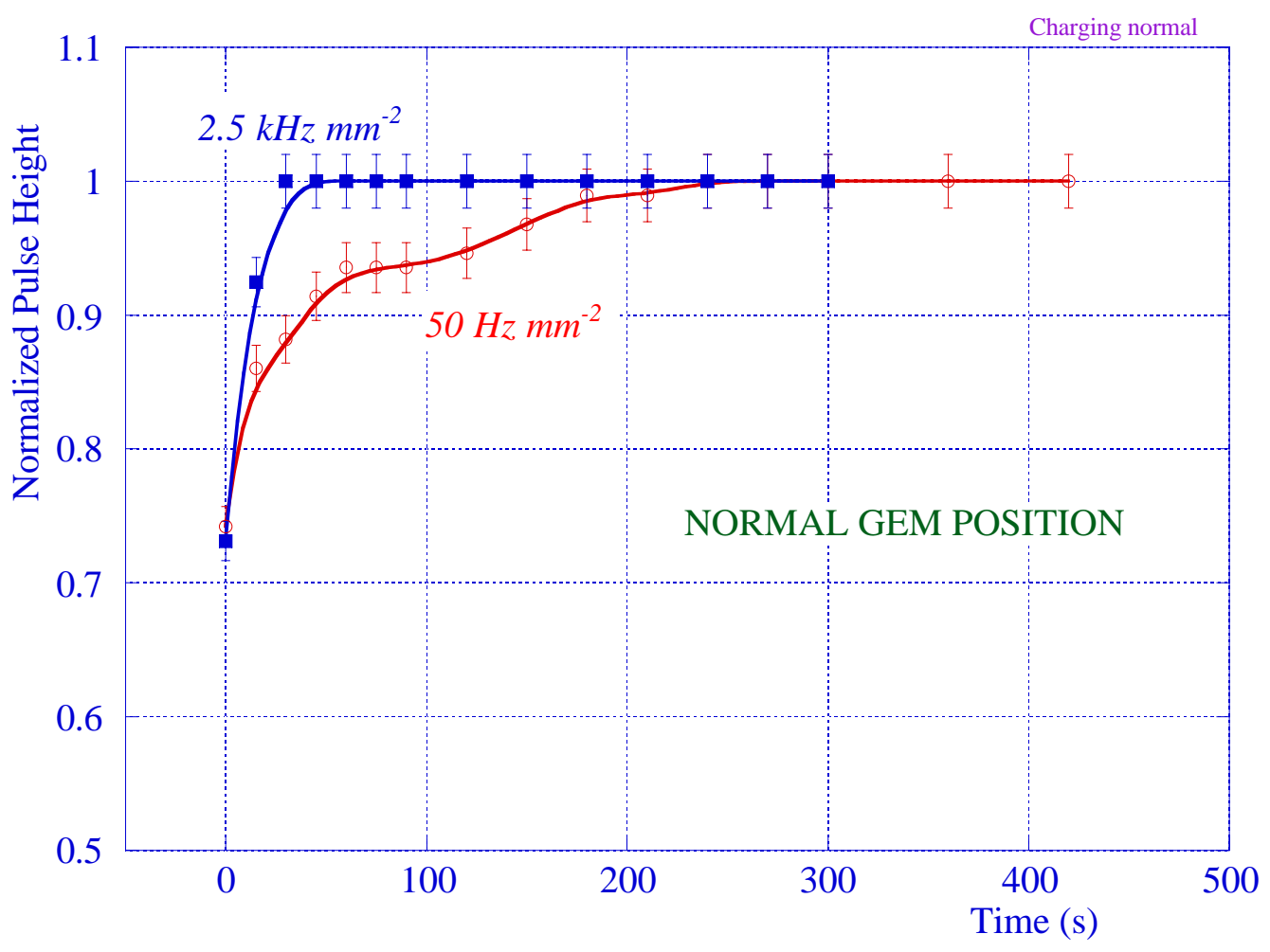

Fig. 30: Gain increase due to local charging-up on irradiation in a normal position.

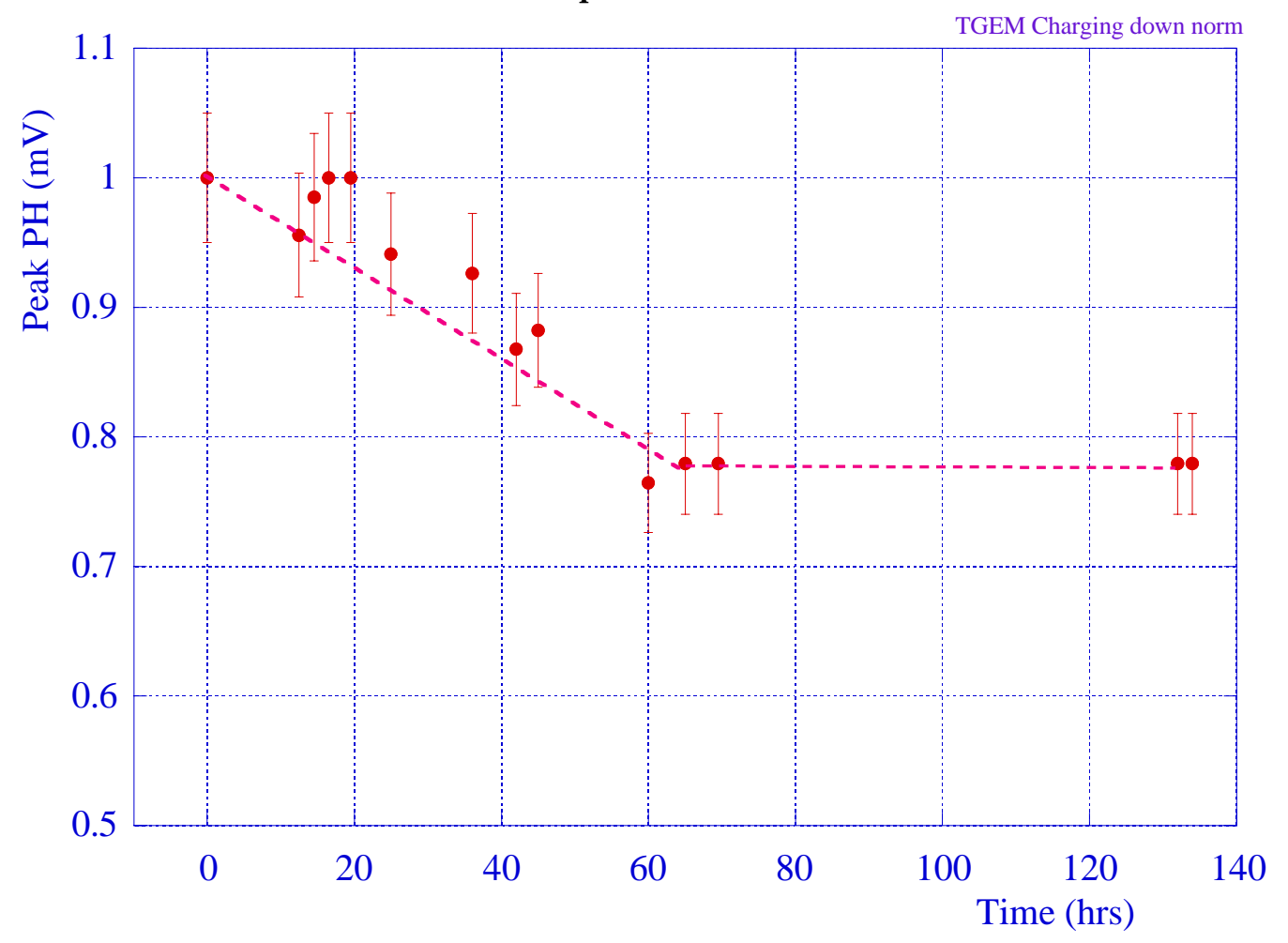

Fig. 31: Long-term charging down in absence of radiation. 


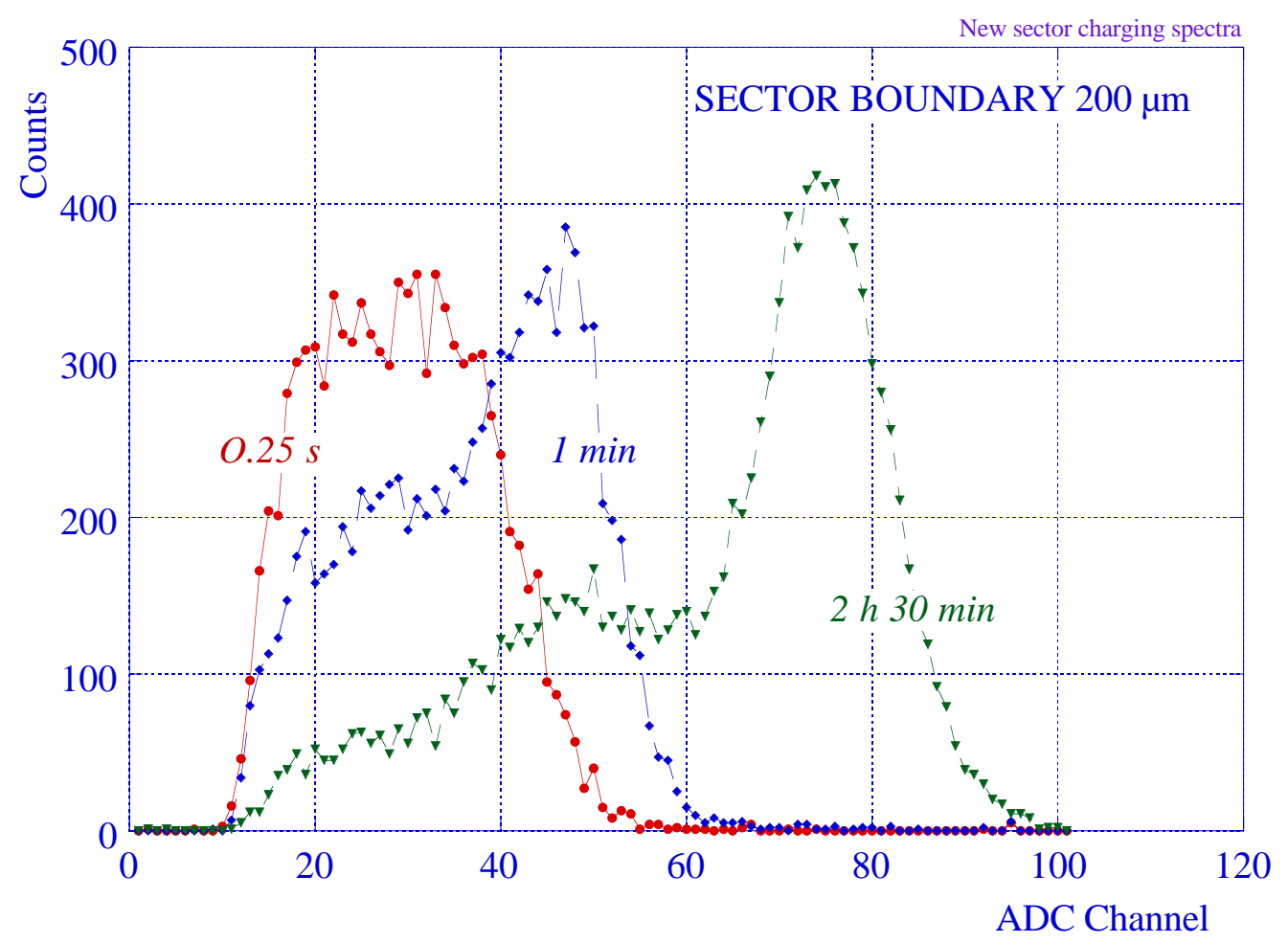

Fig. 32: Evolution of detected pulse height in the sector boundary region.

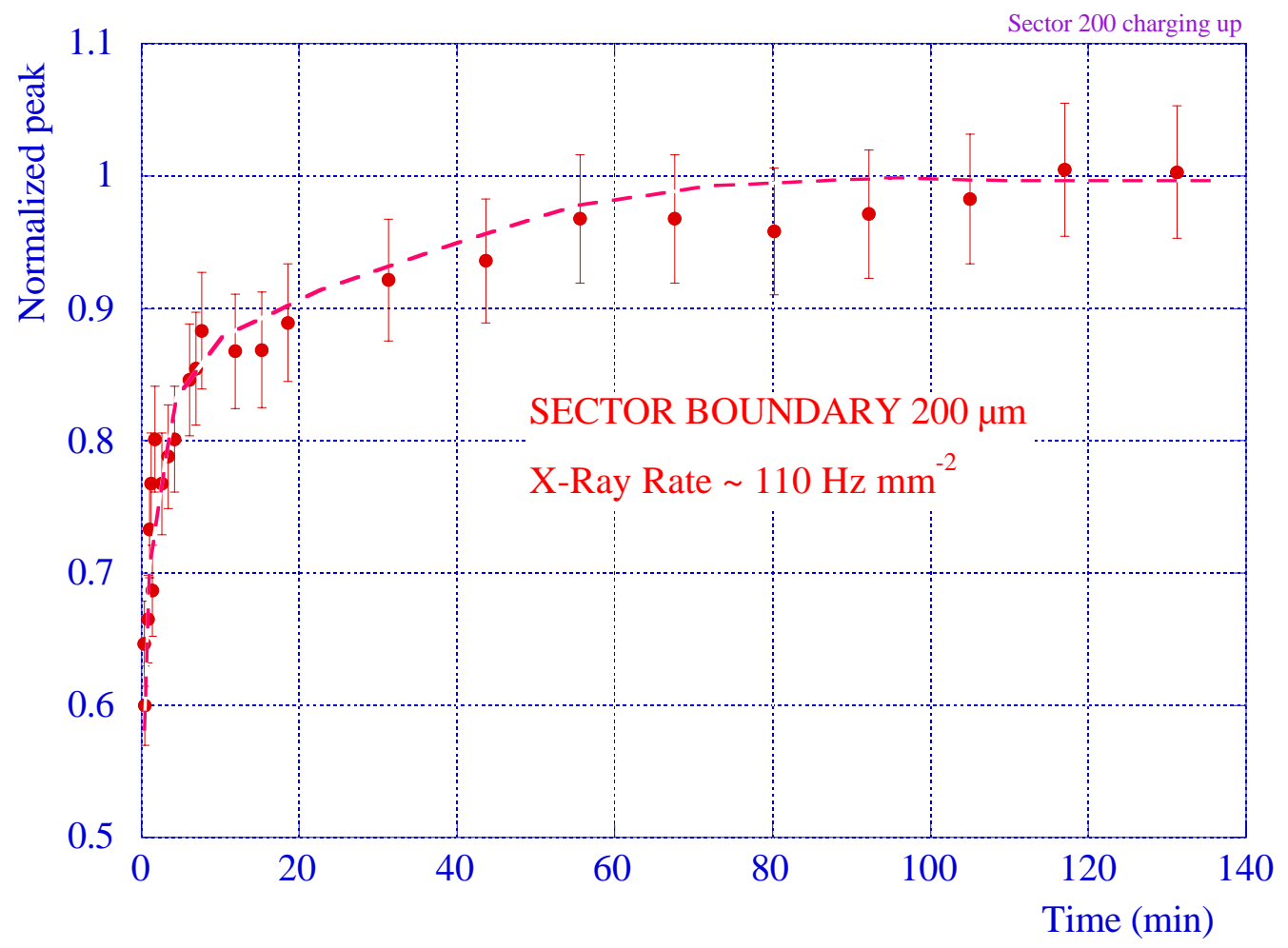

Fig. 33: Sector boundary charging-up. 


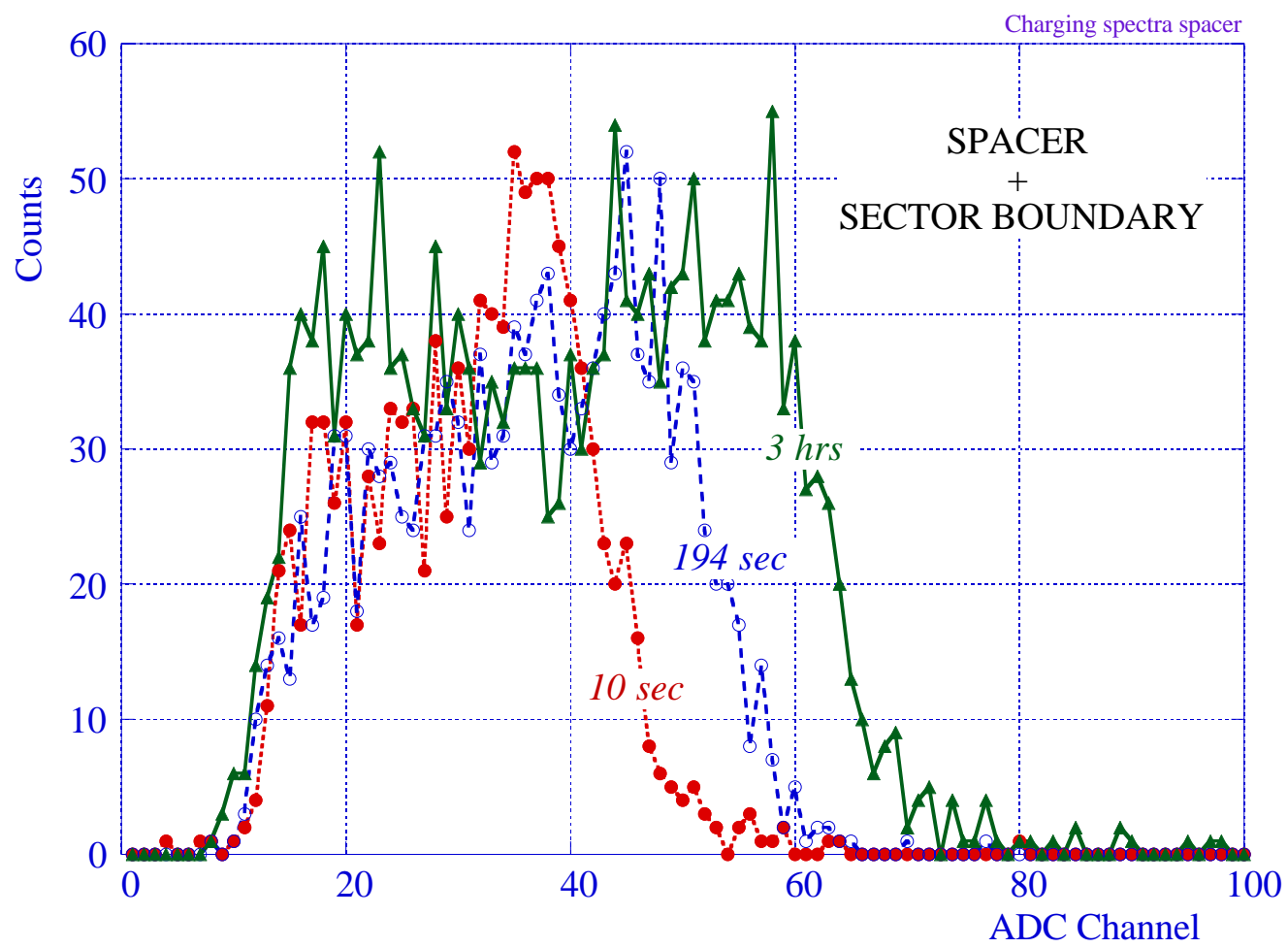

Fig. 34: Evolution of pulse height spectra in the spacer region.

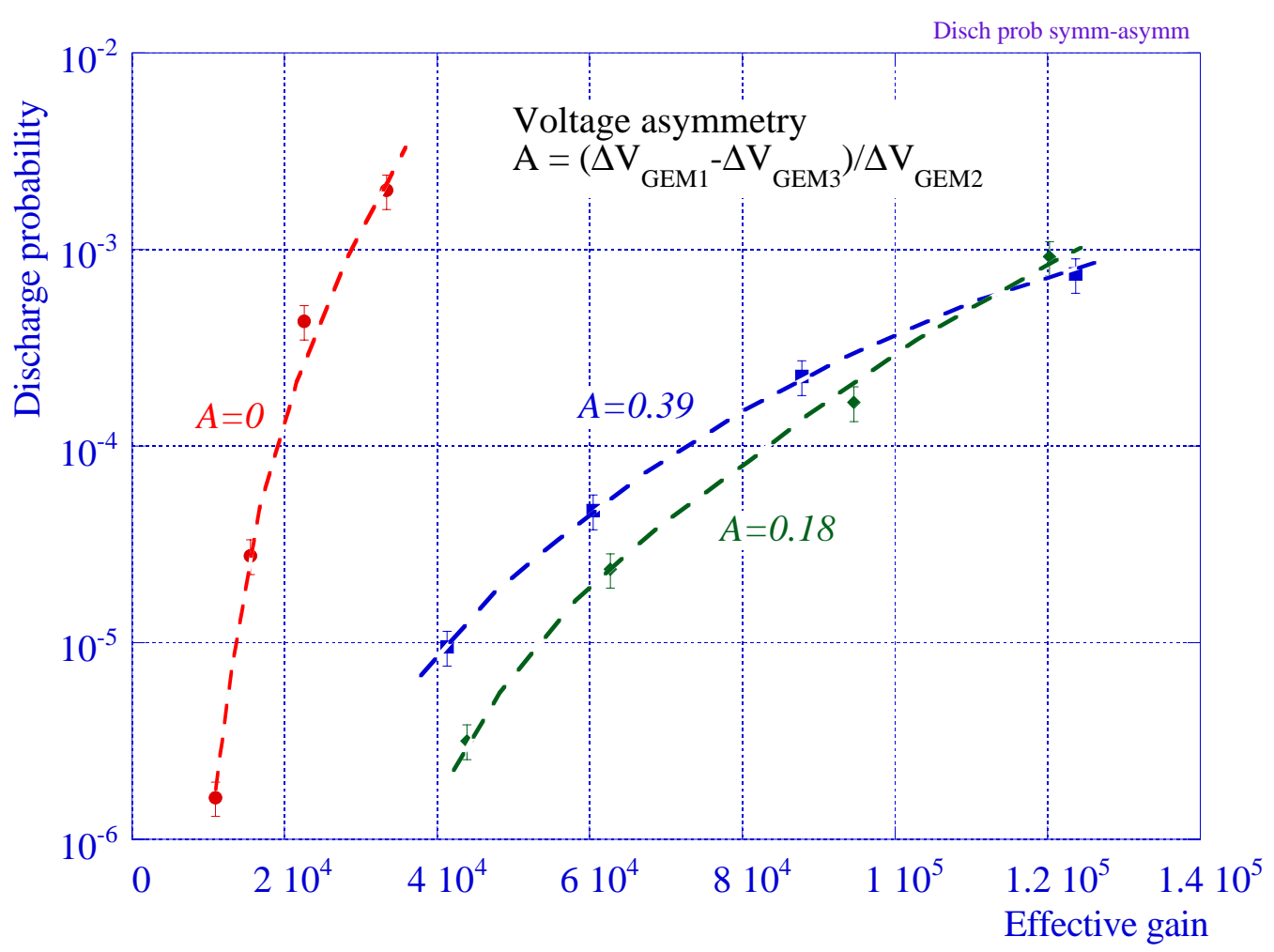

Fig. 35: Discharge probability on irradiation with internal alpha particles as a function of asymmetry in the voltage applied to GEMs. Note that the gain range investigated largely exceeds the normal operating value (below $10^{4}$ ). 


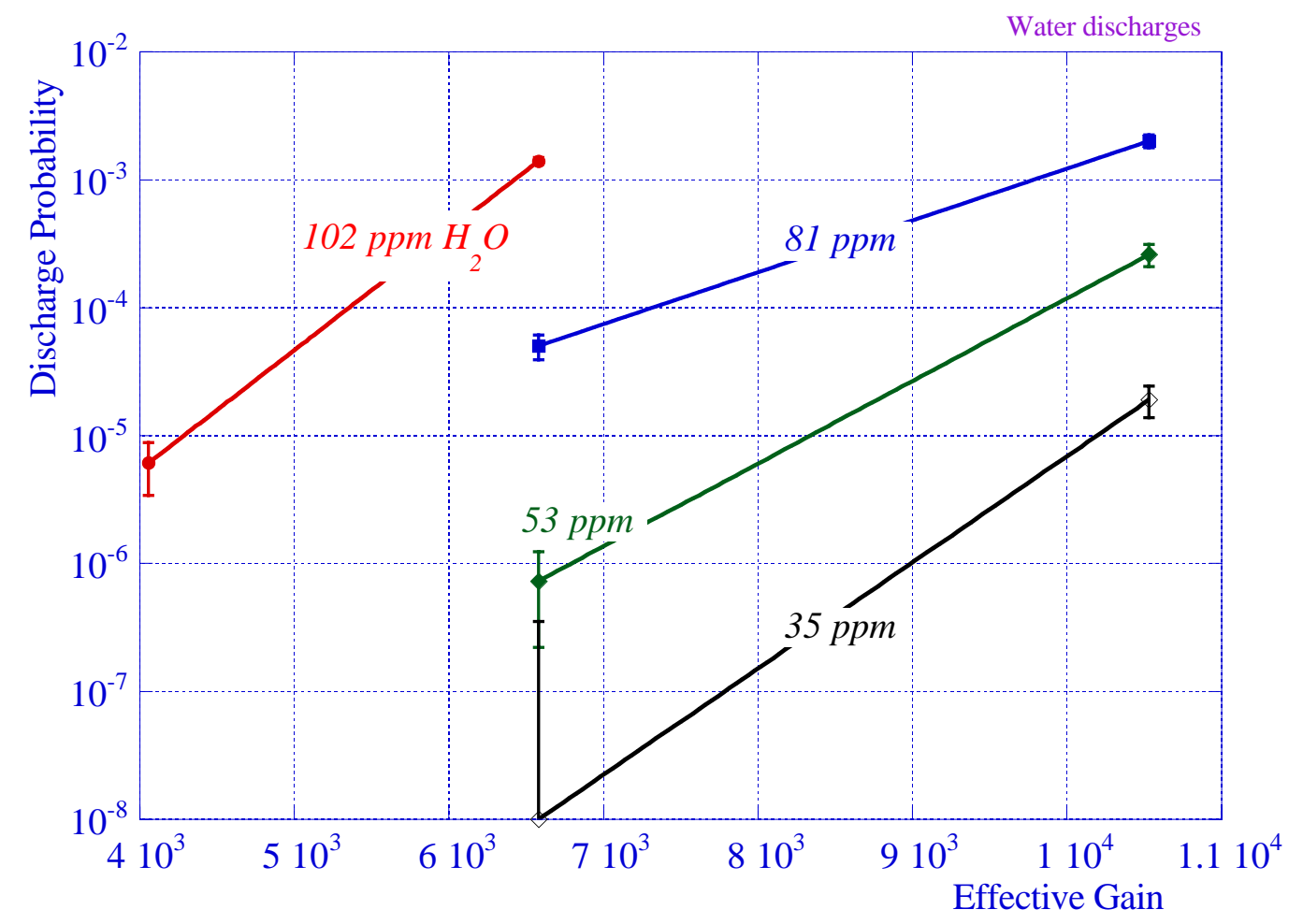

Fig. 36: Discharge probability on alphas as a function of moisture level in the gas.

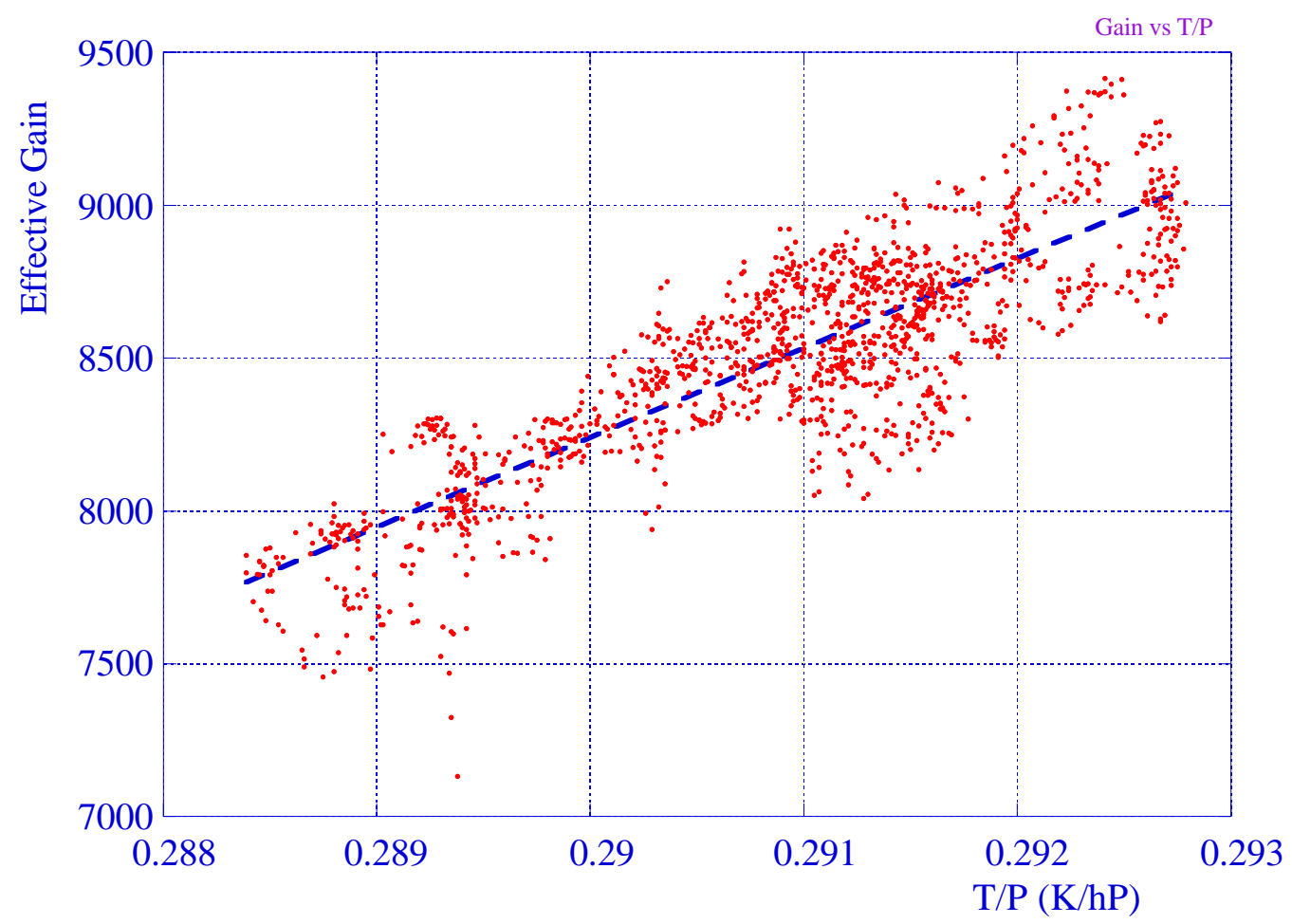

Fig. 37: Effective gain as a function of the ratio temperature to pressure. 


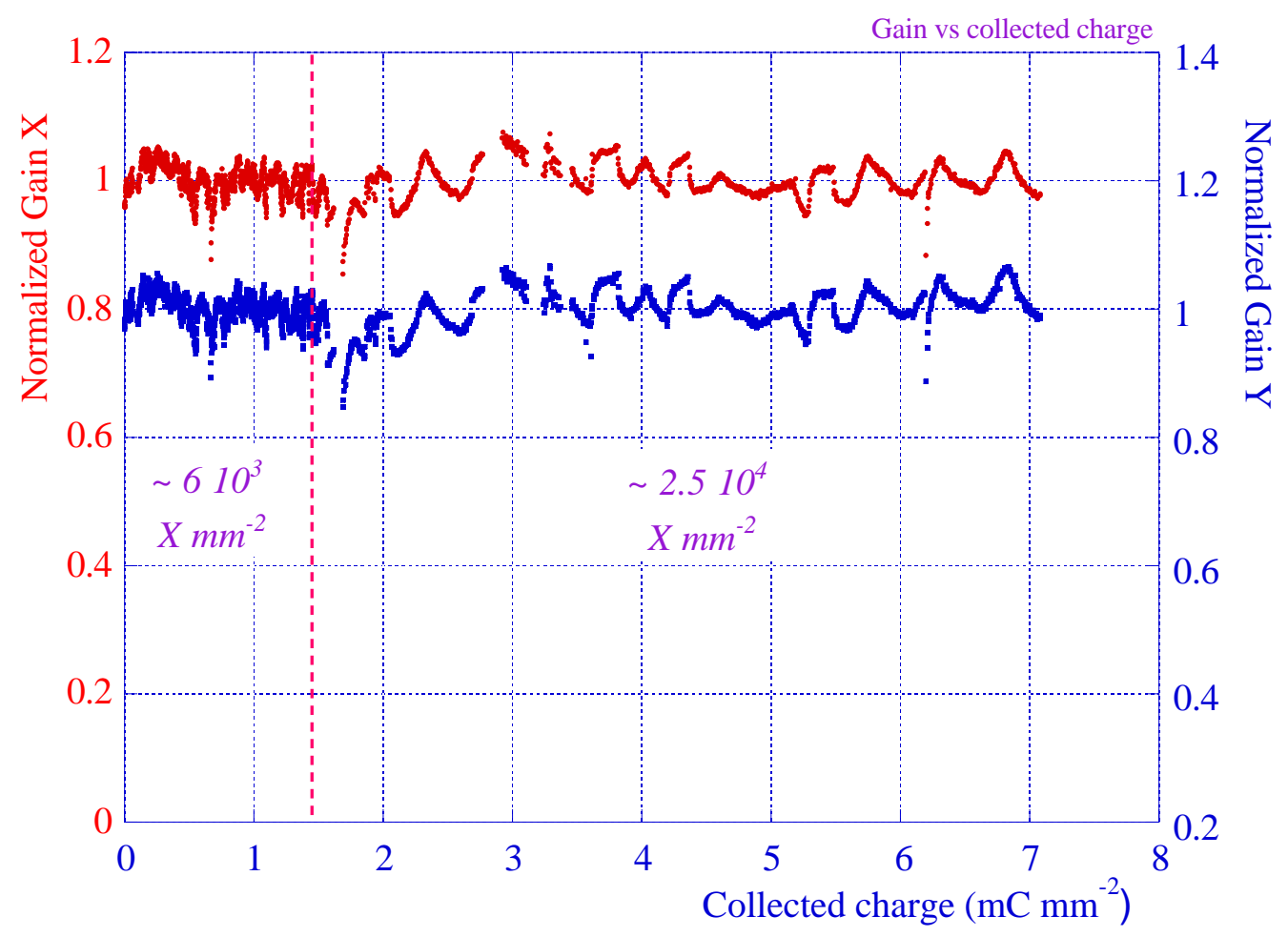

Fig. 38: Long-term gain stability under irradiation.

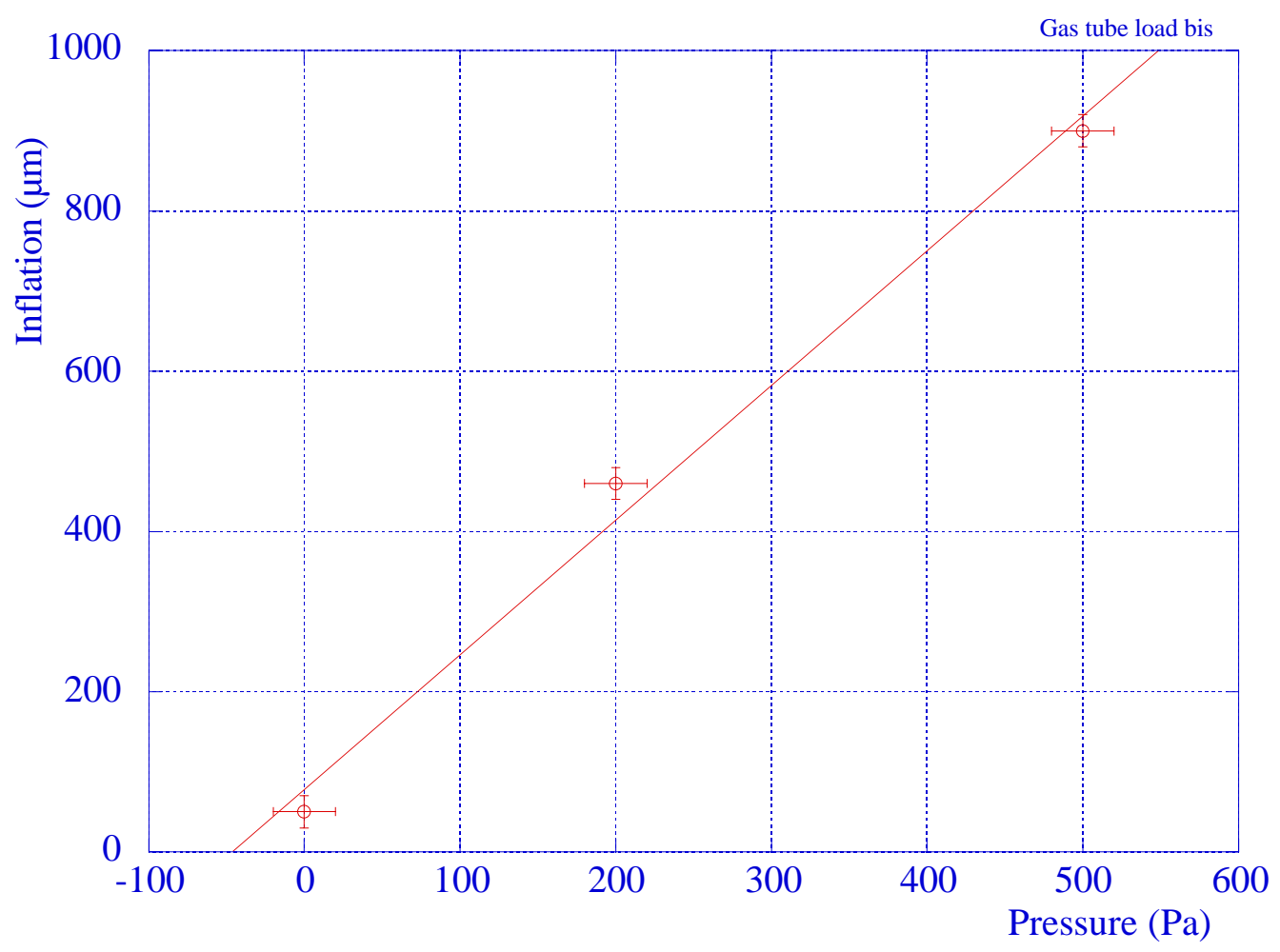

Fig. 39: Detector inflation in the center as a function of over-pressure. 


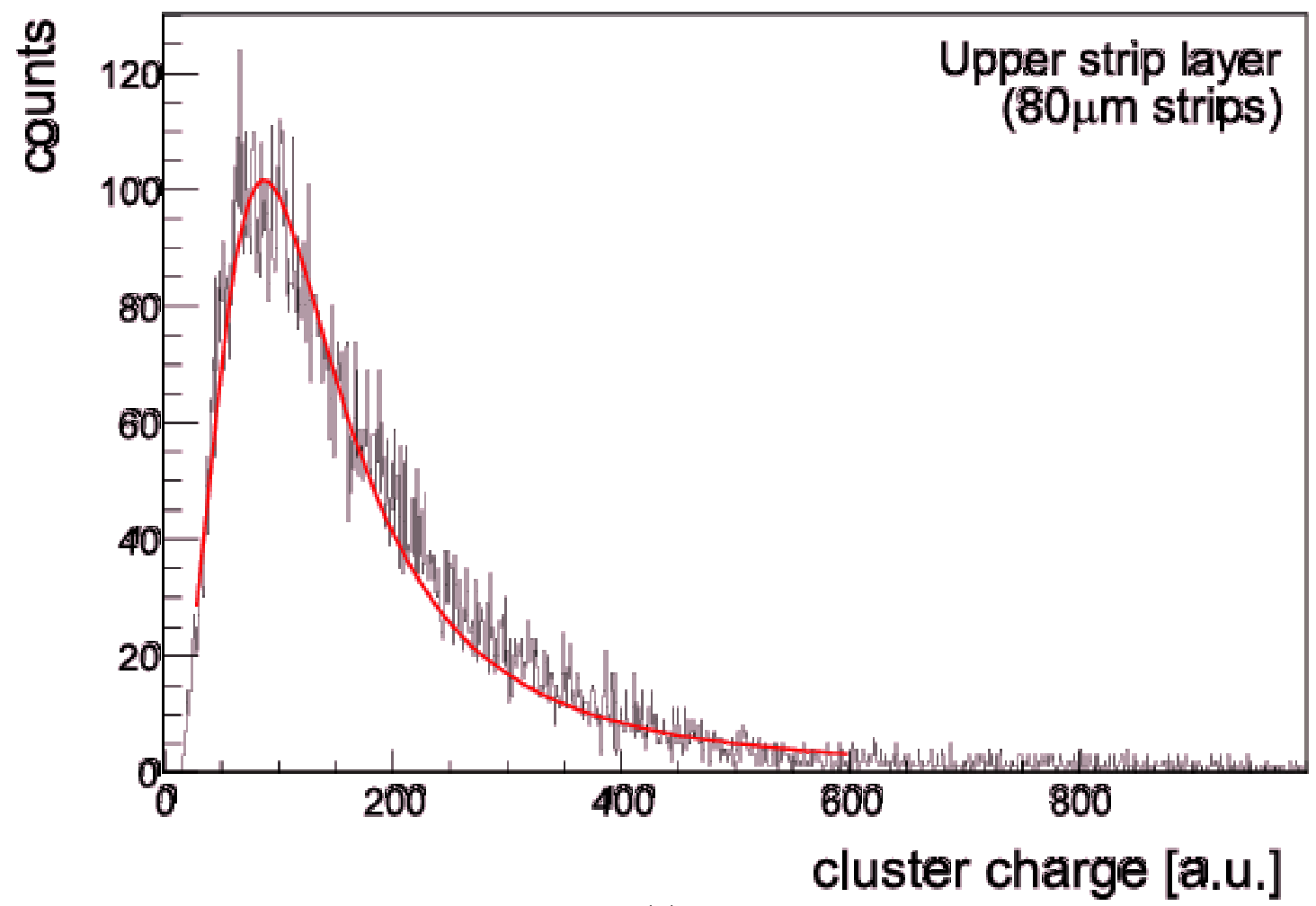

(a)

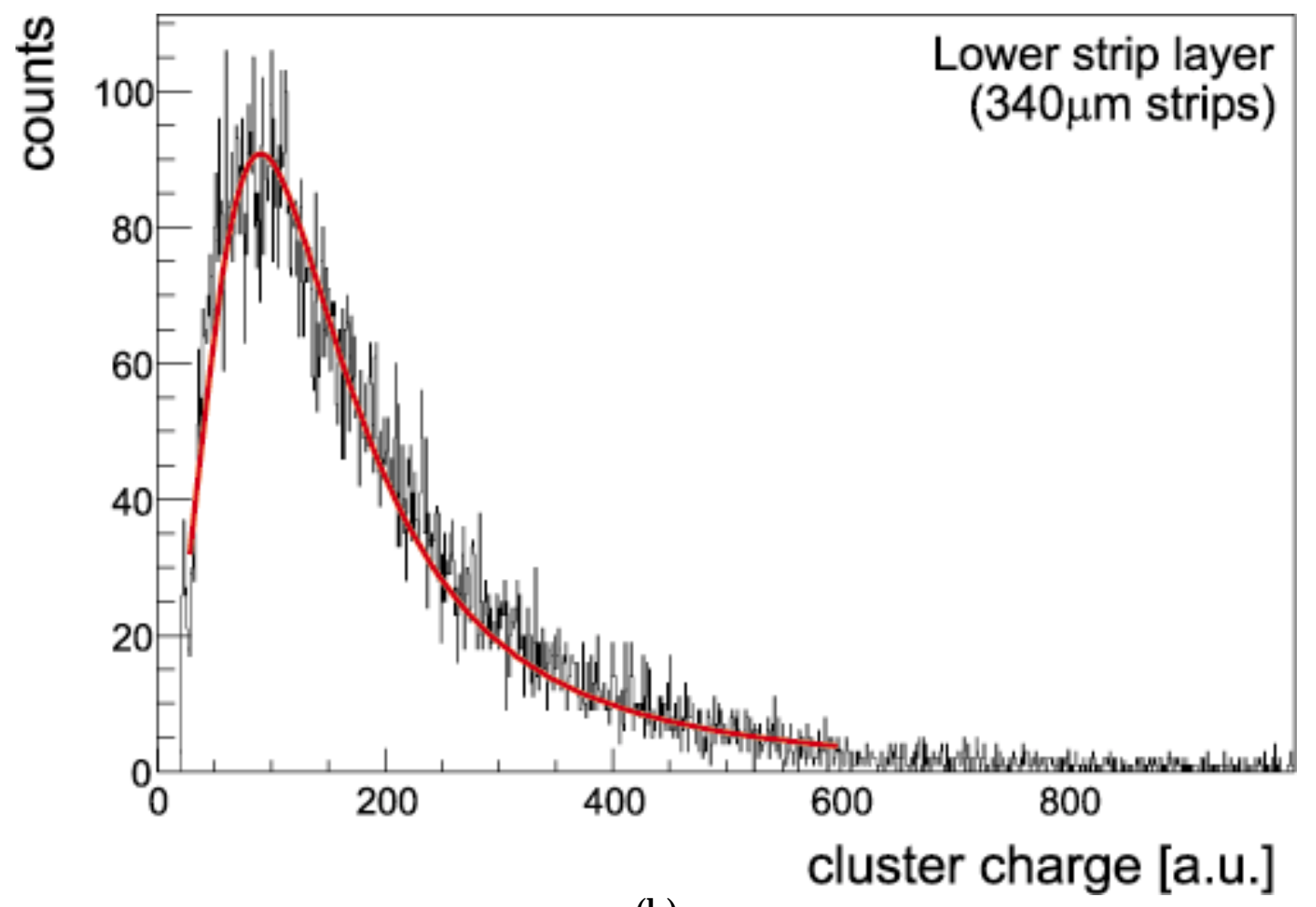

(b)

Fig. 40: Cluster charge distribution for minimum ionizing particles in the $\mathrm{x}(\mathrm{a})$ and $\mathrm{y}(\mathrm{b})$ projection. 


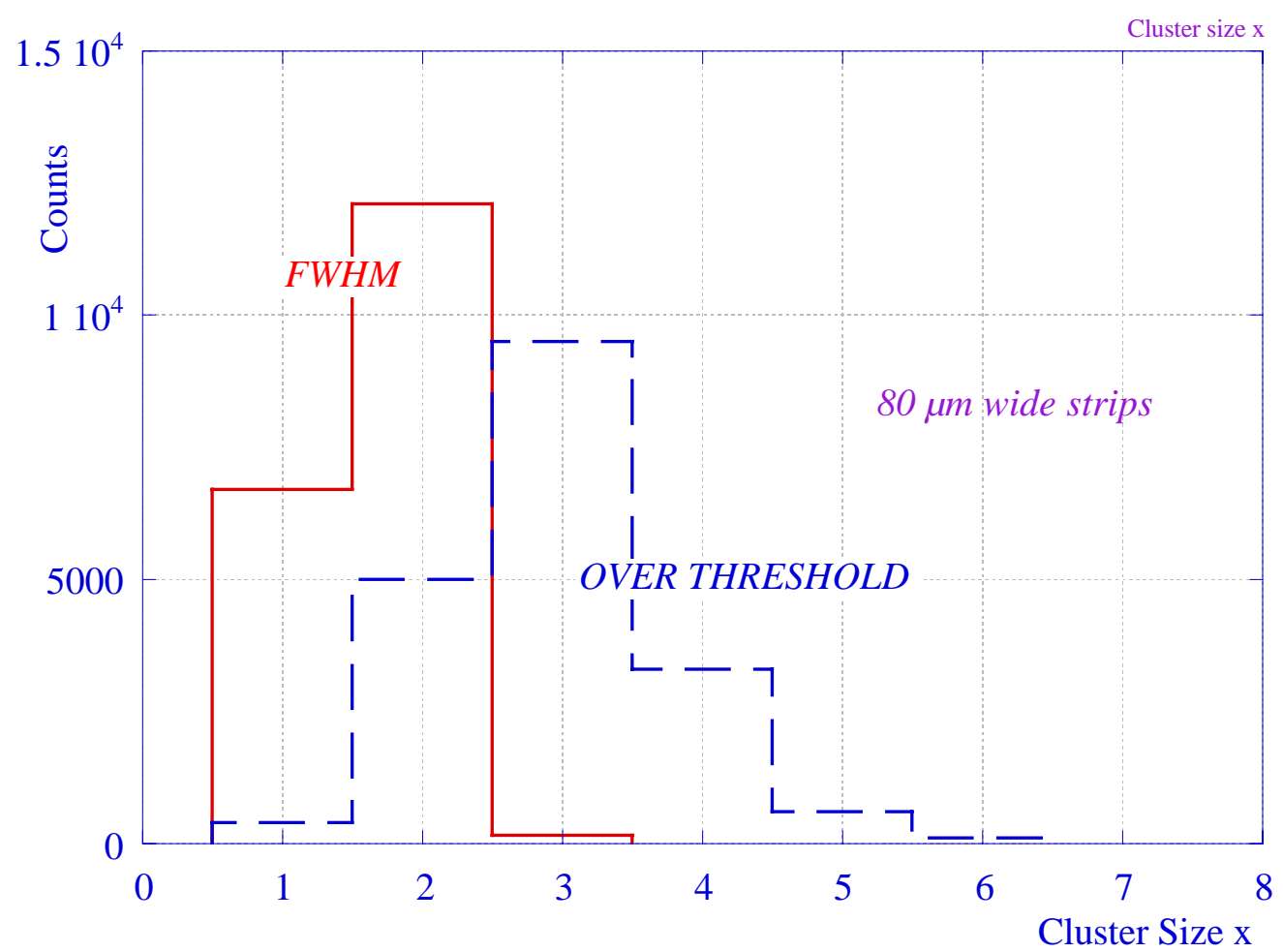

(a)

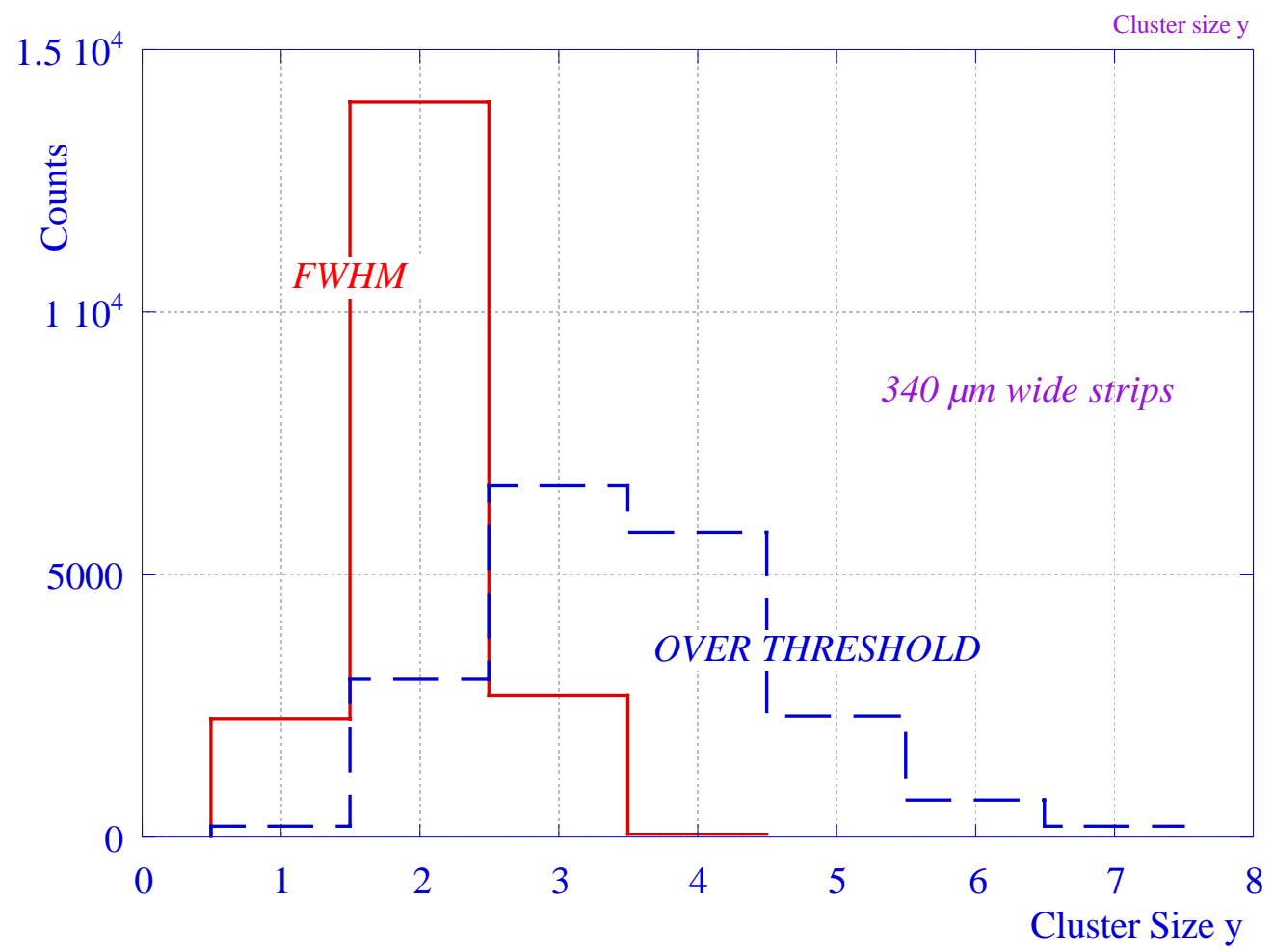

(b)

Fig. 41: Cluster size, in number of strips, for the $\mathrm{x}(\mathrm{a})$ and $\mathrm{y}(\mathrm{b})$ projections; full curve: FWHM, dashed lines: channels above threshold. The pitch is $400 \mu \mathrm{m}$. 


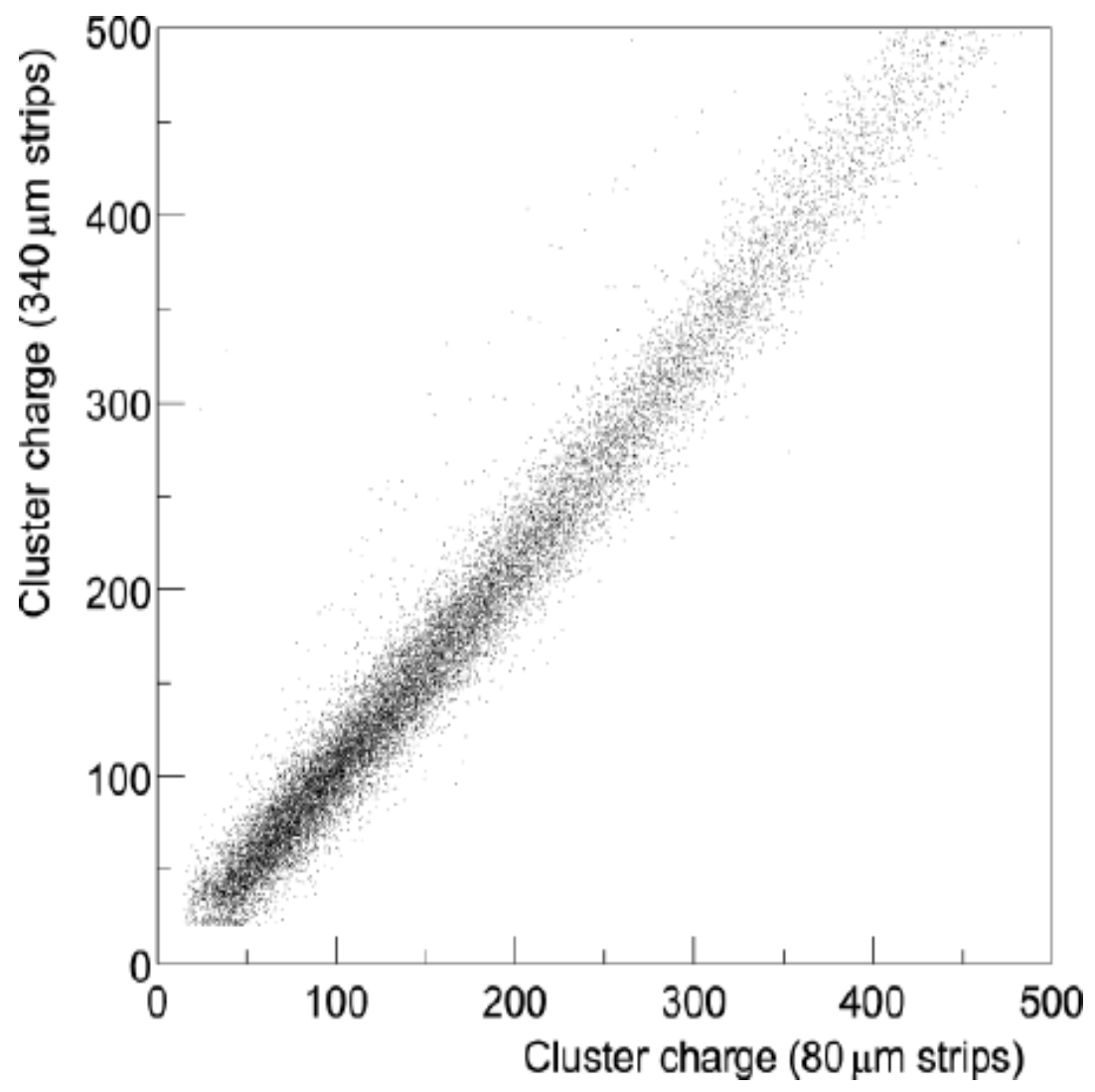

Fig. 42: Cluster charge correlation between the two coordinates.

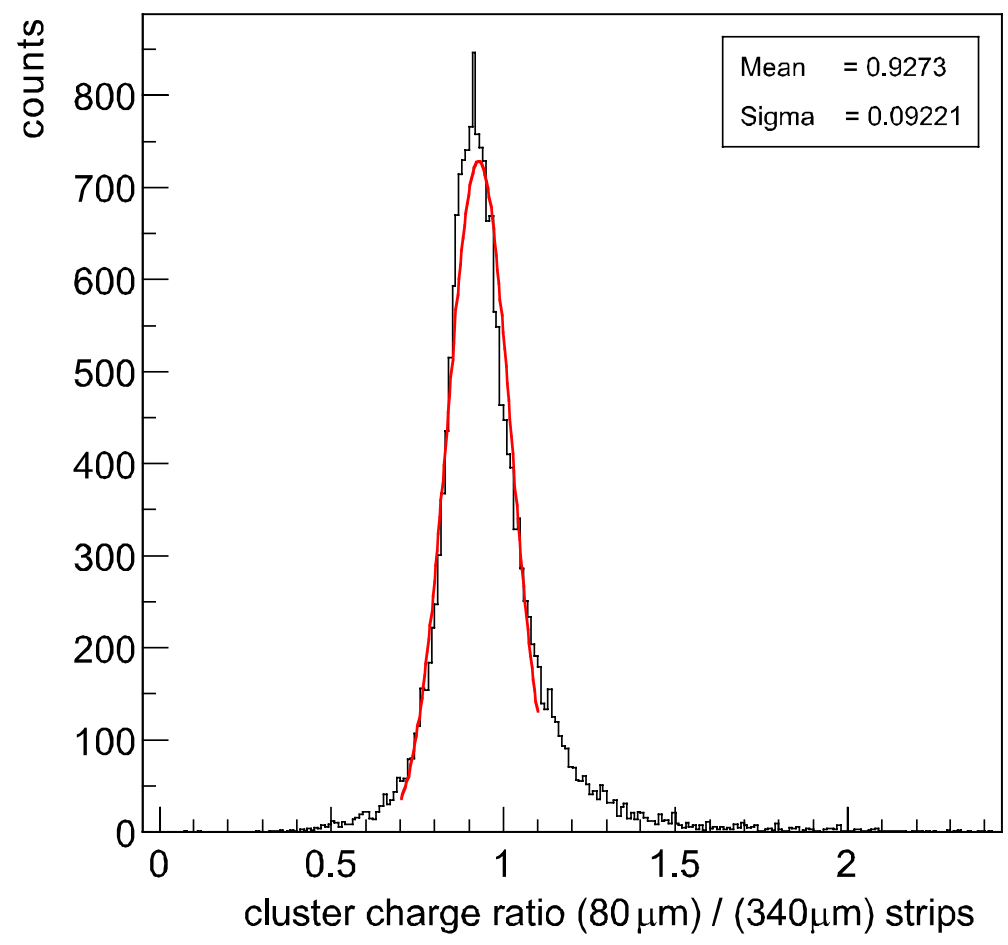

Fig. 43: Cluster charge ratio between $\mathrm{x}$ and $\mathrm{y}$. 


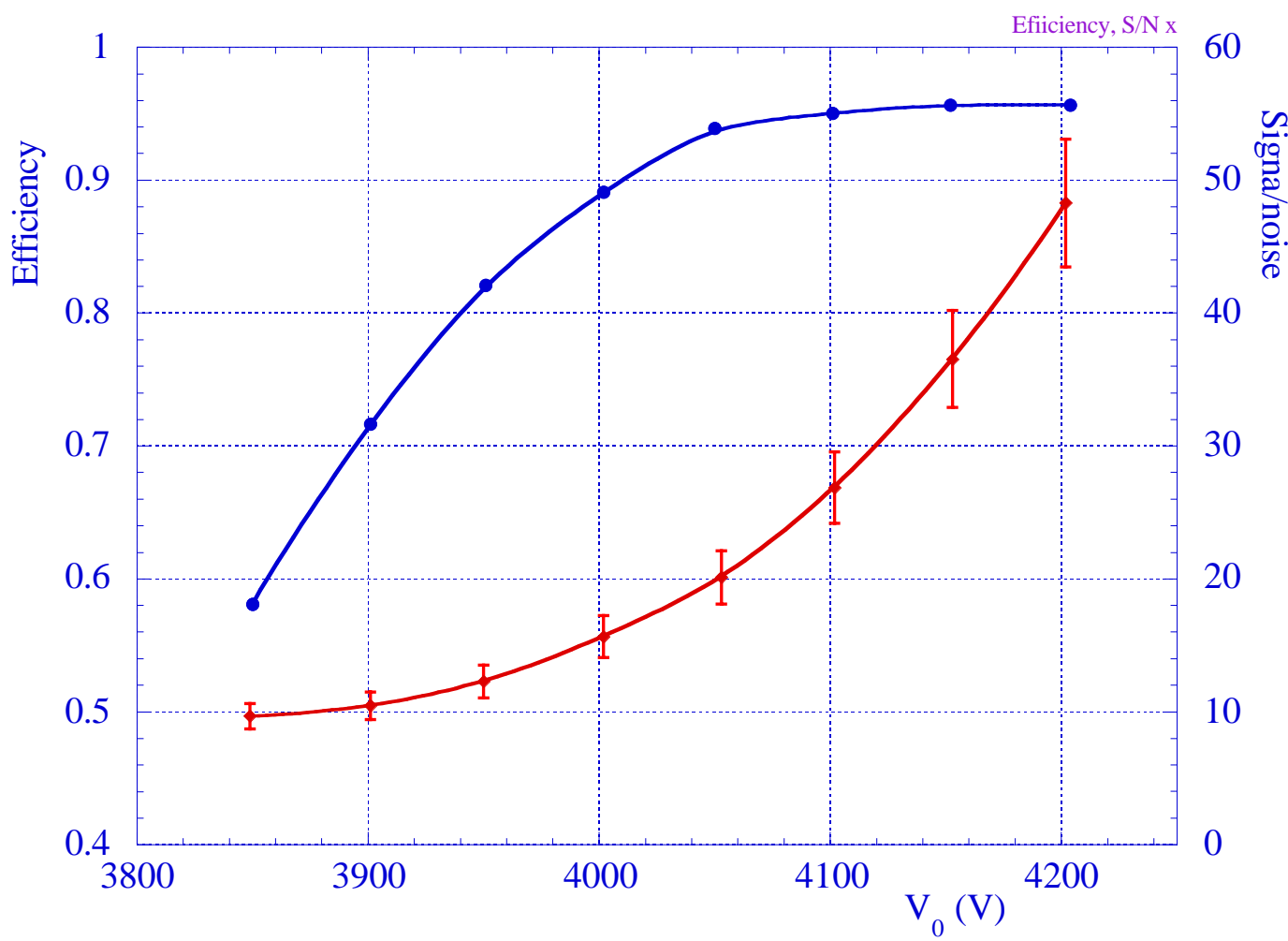

(a)

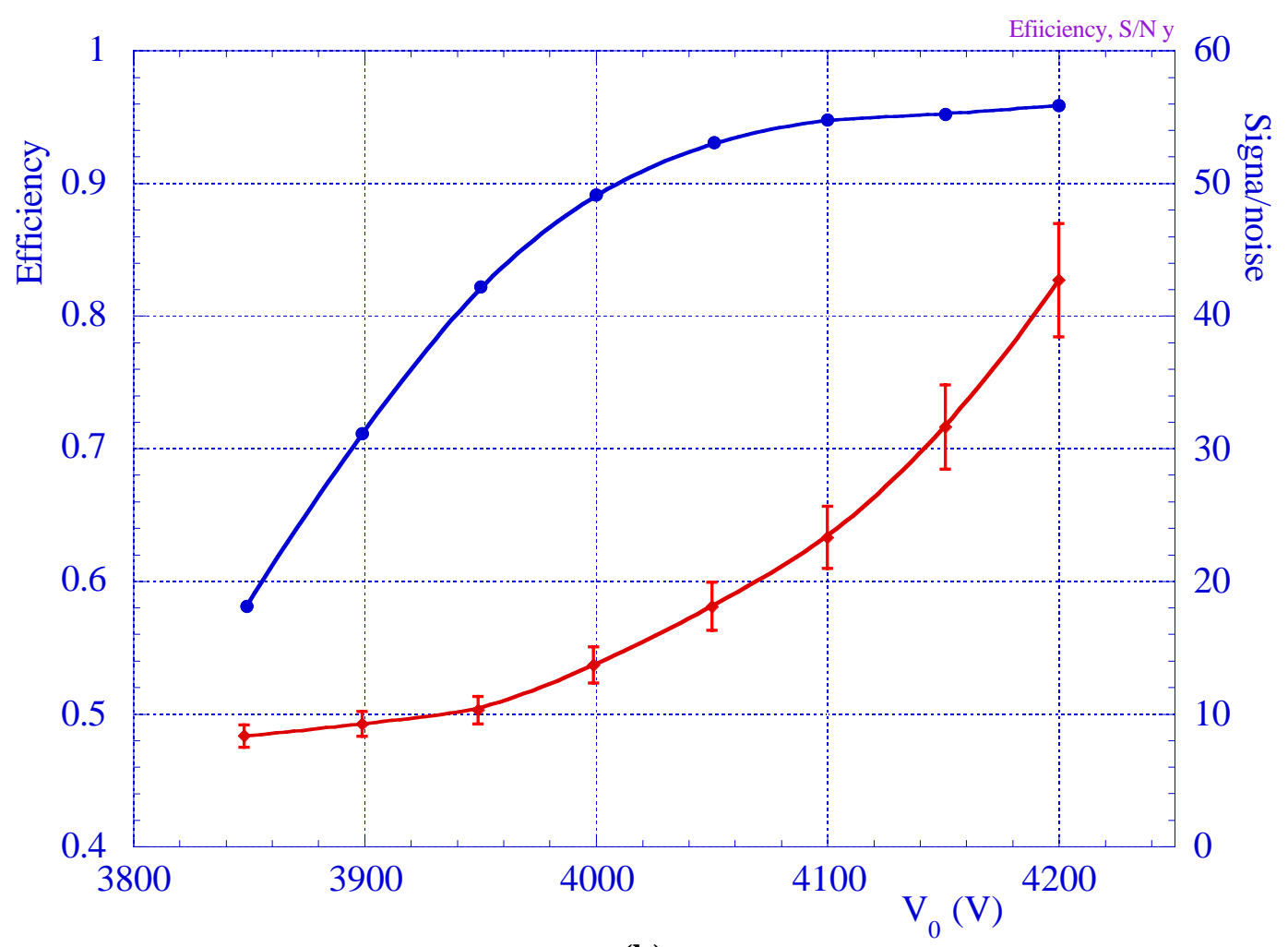

(b)

Fig. 44: Efficiency and signal/noise for minimum ionizing particles for the $x$ and y projections as a function of operating voltage. 


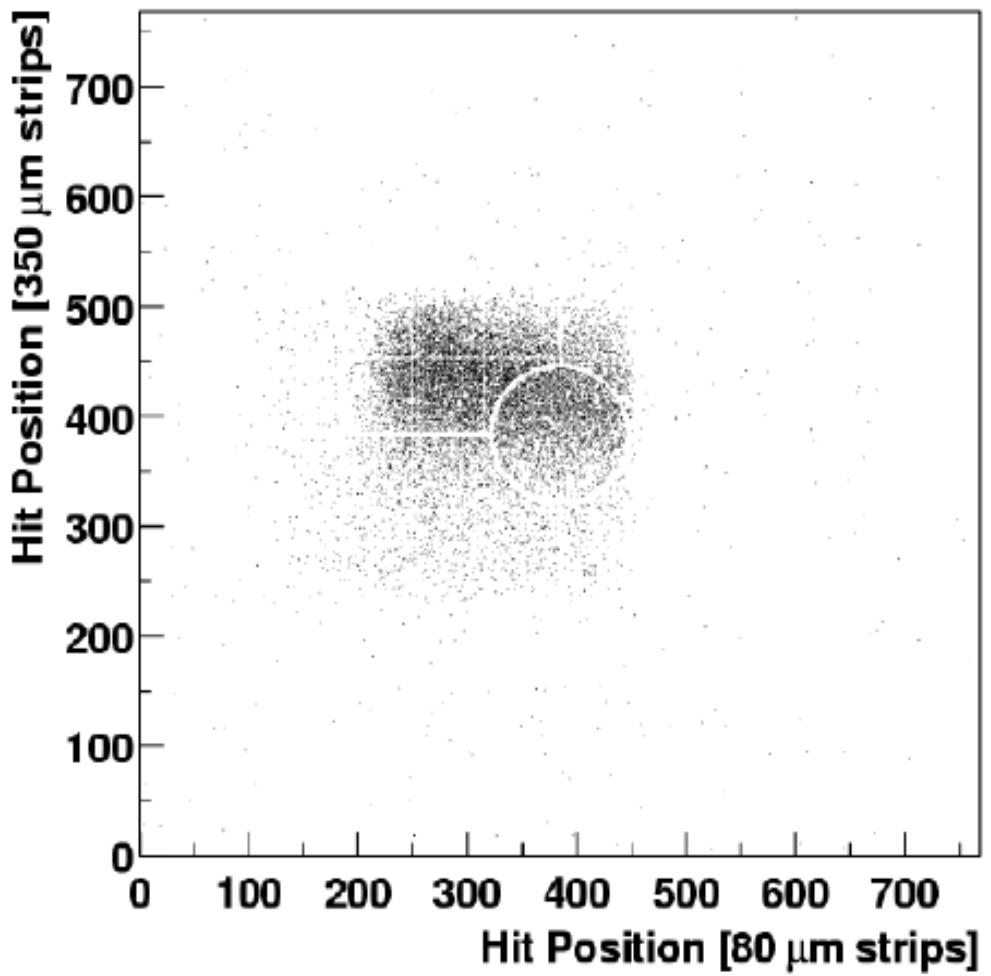

(a)

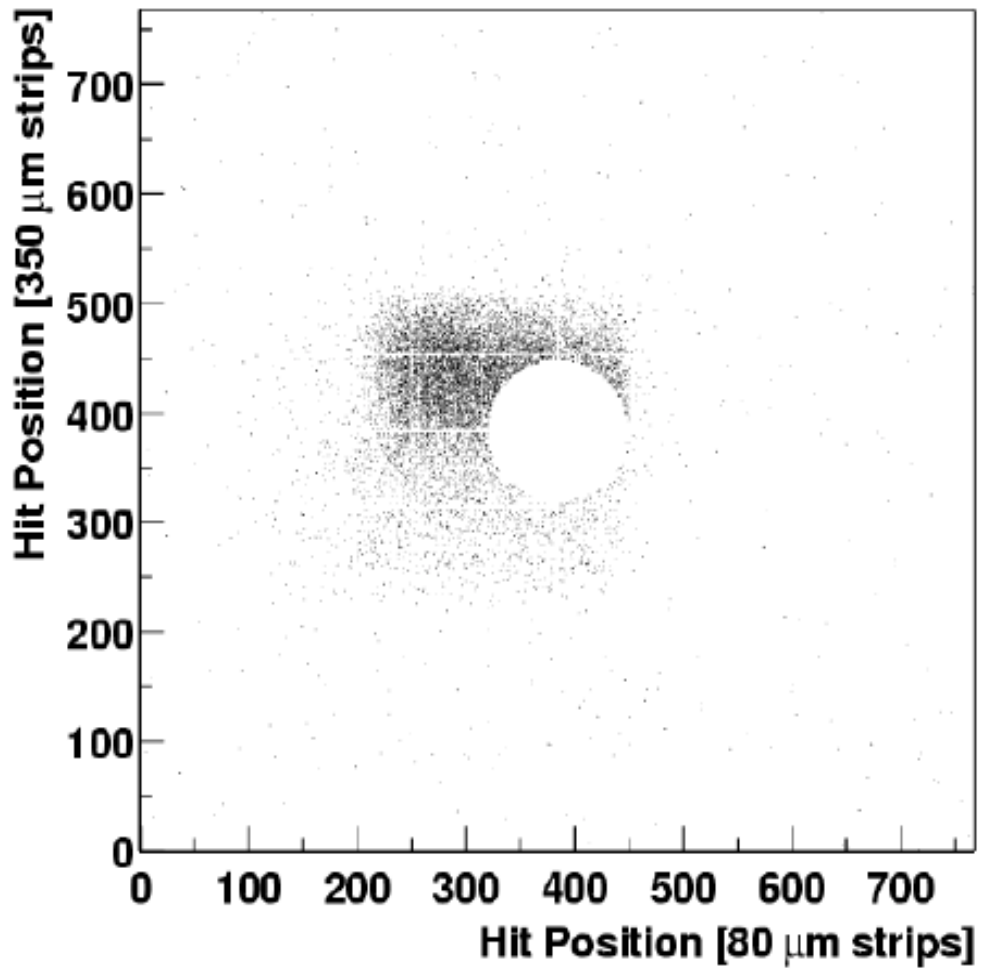

(b)

Fig. 45: Two-dimensional position distribution of tracks plot without (a) and with (b) the beam killer activated. 


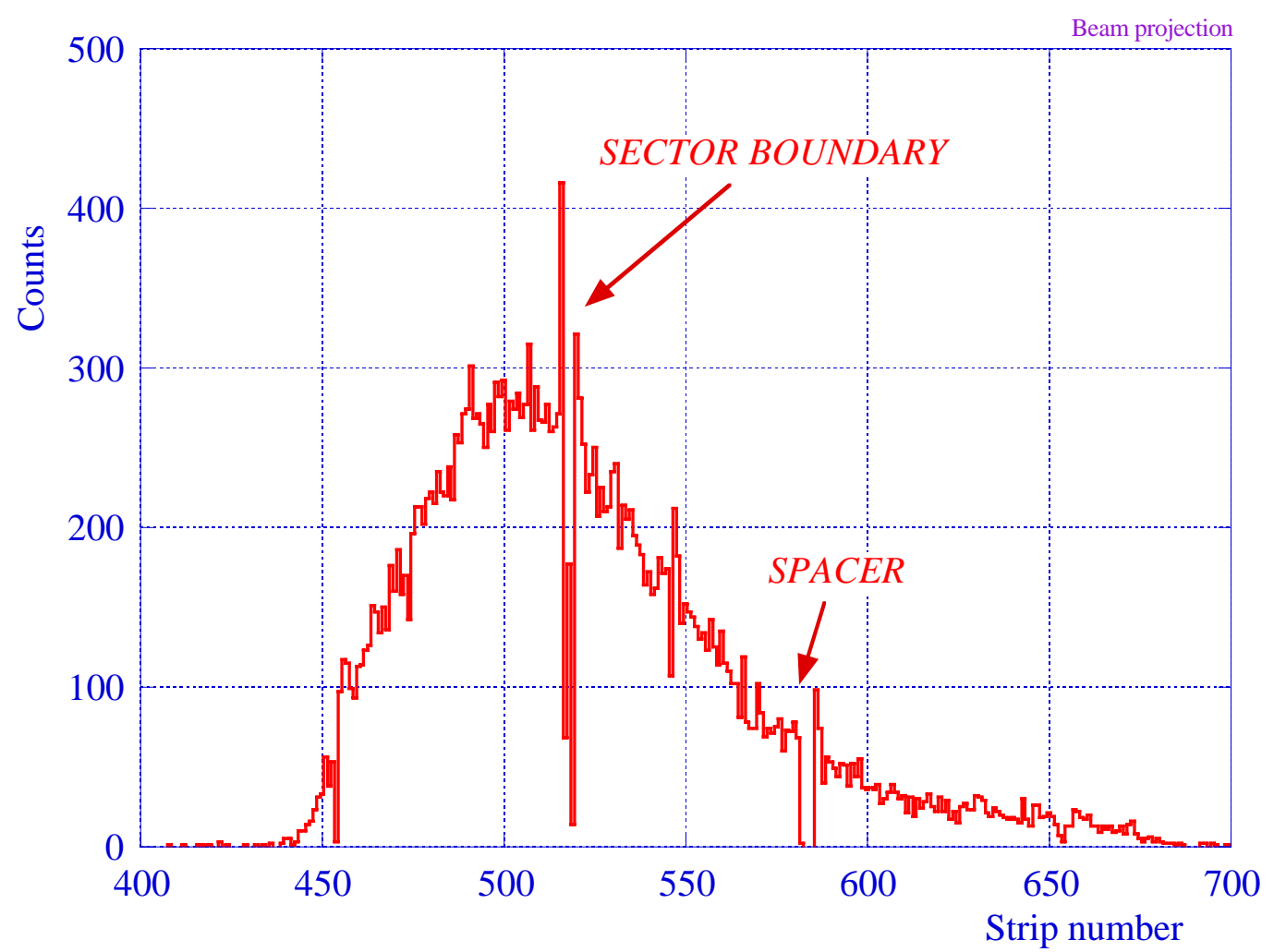

Fig. 46: Projection of beam hits along one coordinate, showing local losses due to a space and a sector boundary.

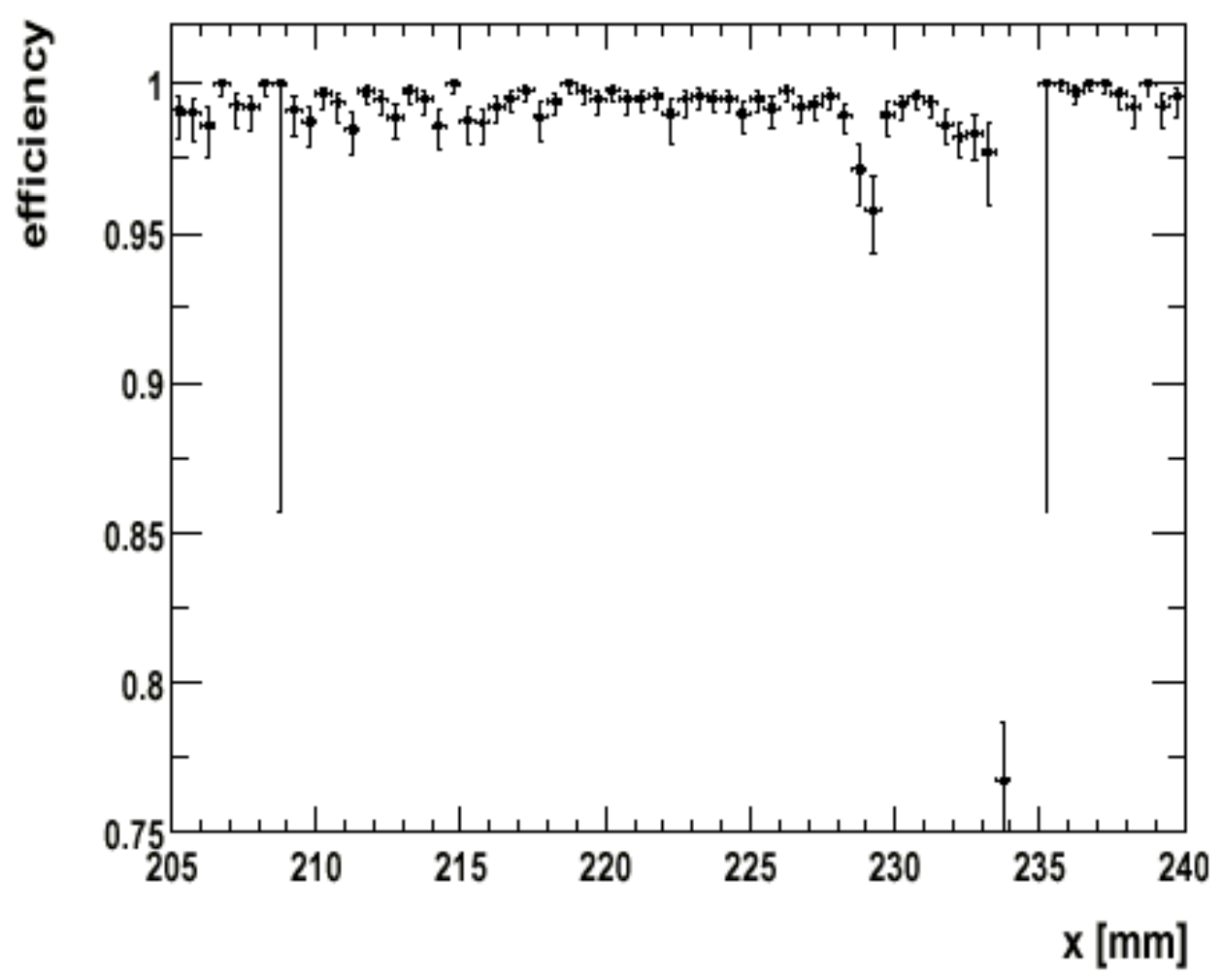

Fig. 47: Efficiency as a function of the $\mathrm{x}$ coordinate showing local losses due to spacers and sector boundaries. 


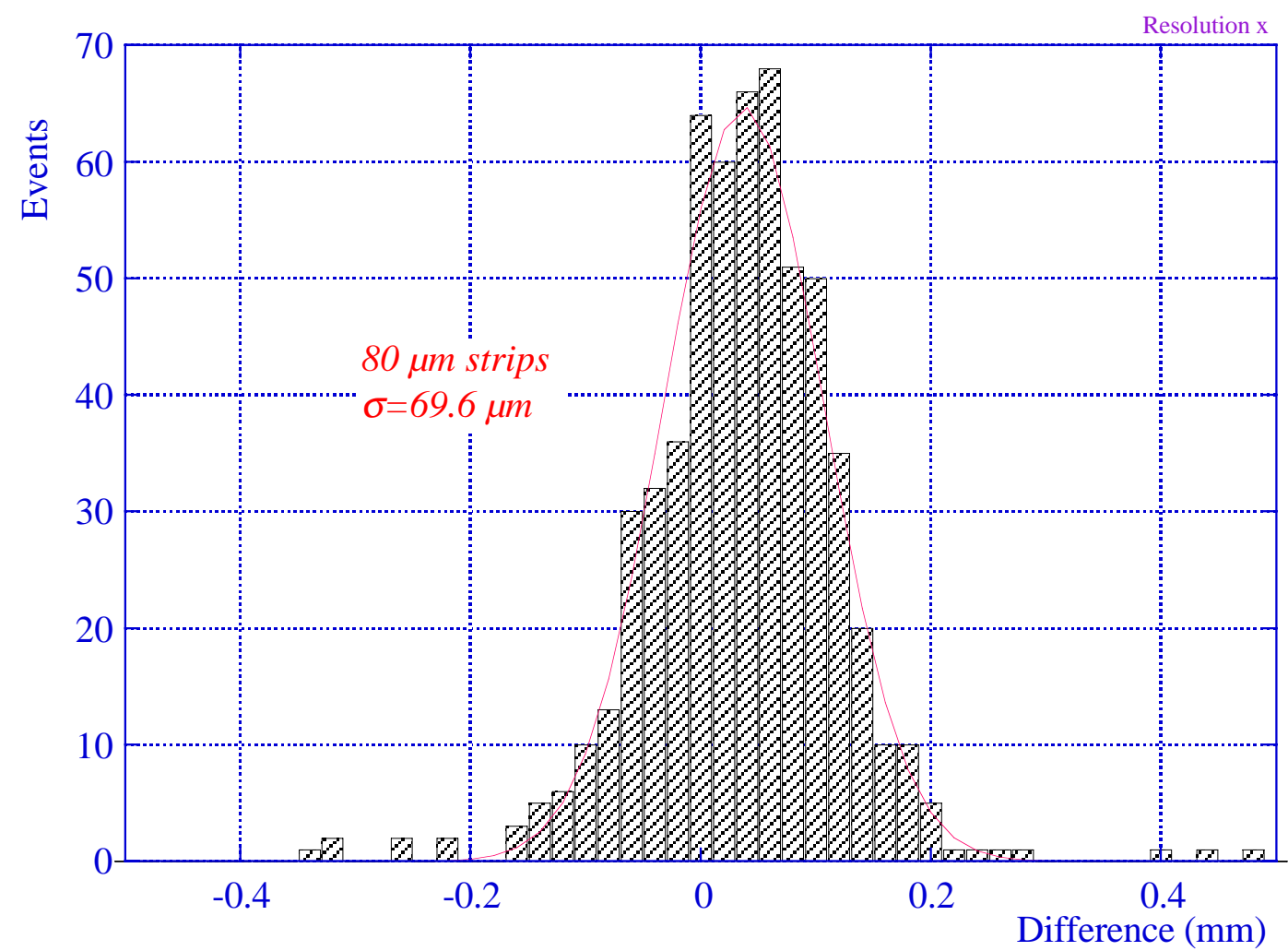

(a)

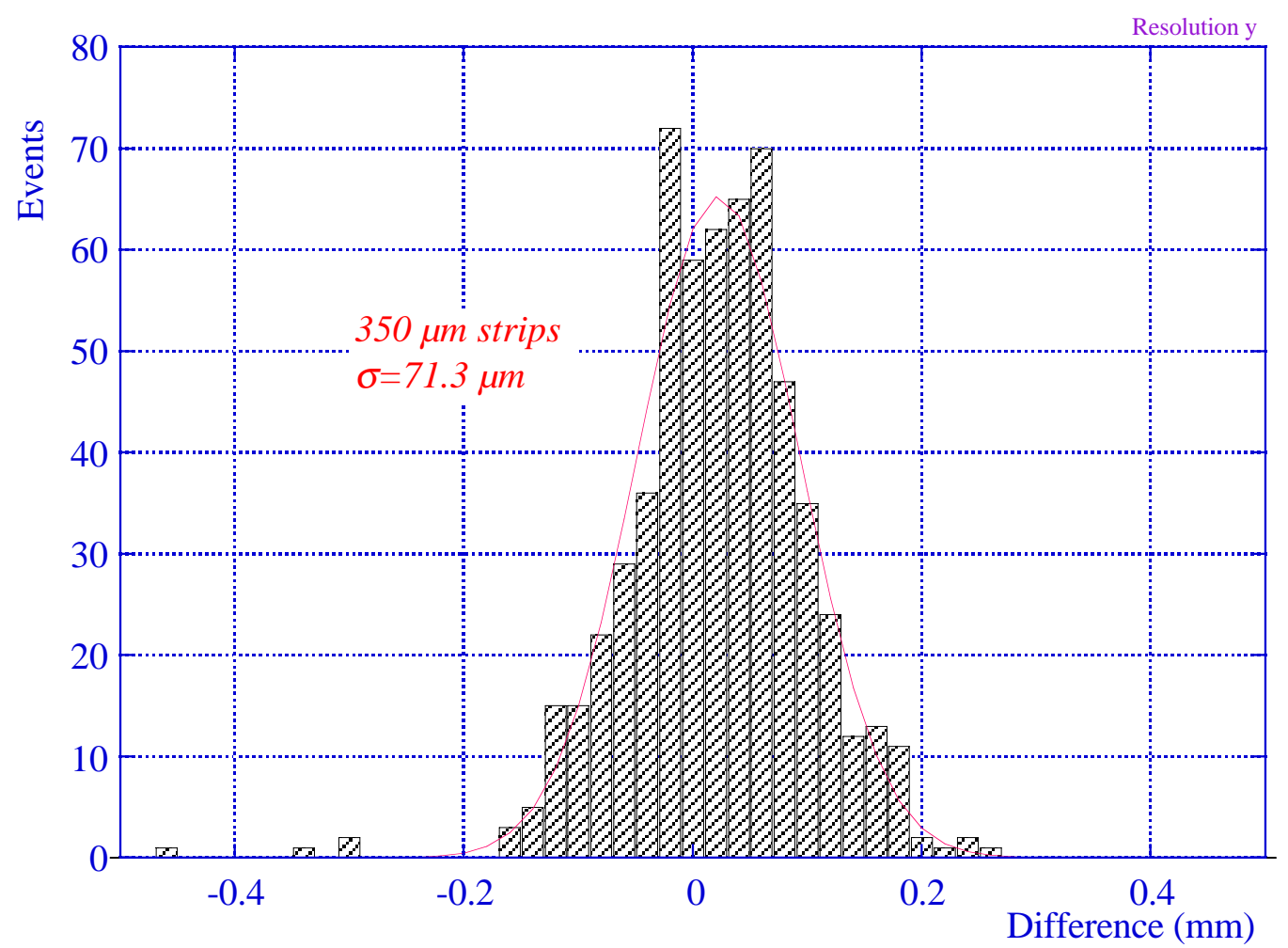

(b)

Fig. 48: Difference of coordinates measured for the $\mathrm{x}(\mathrm{a})$ and $\mathrm{y}(\mathrm{b})$ projections. 


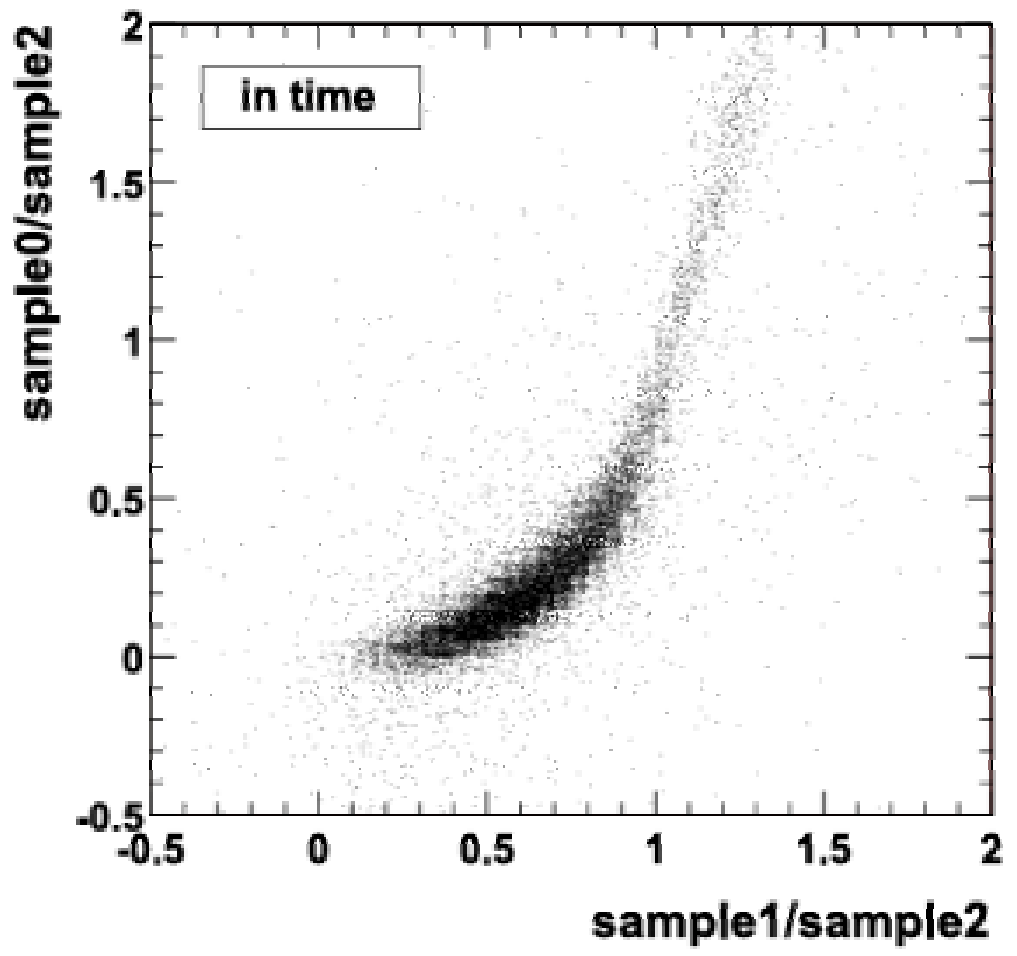

Fig. 49: Timing plot.

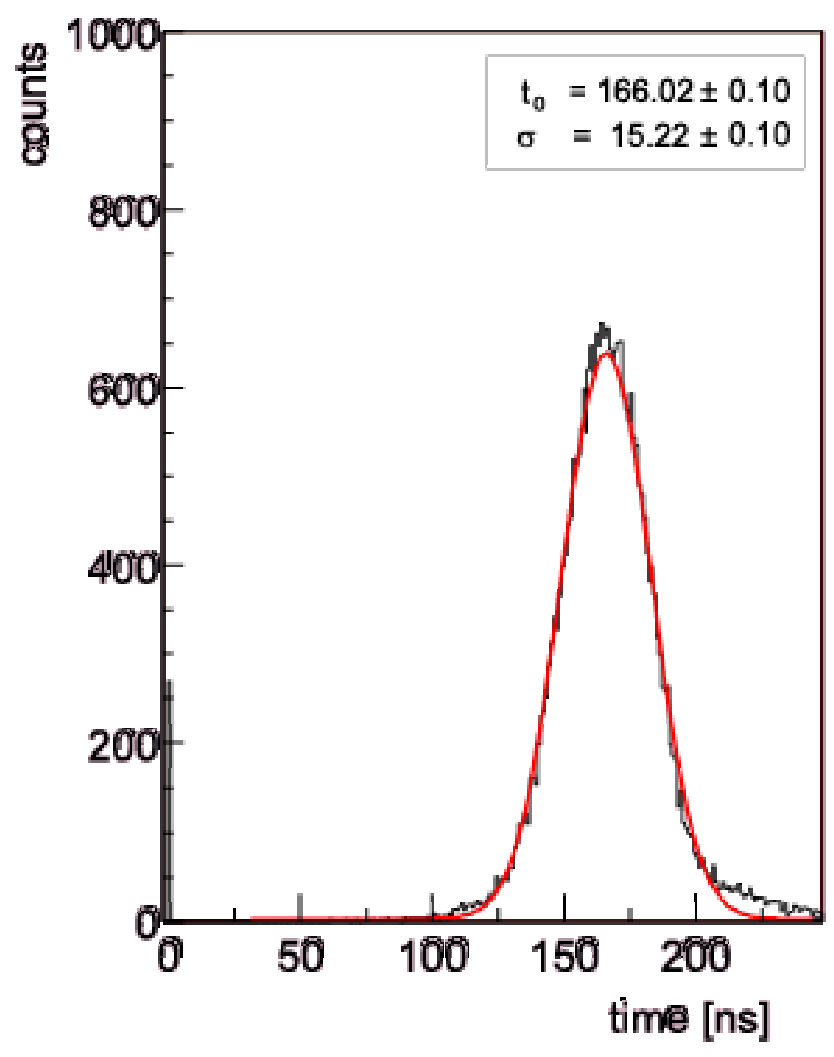

Fig. 50: Time resolution. 


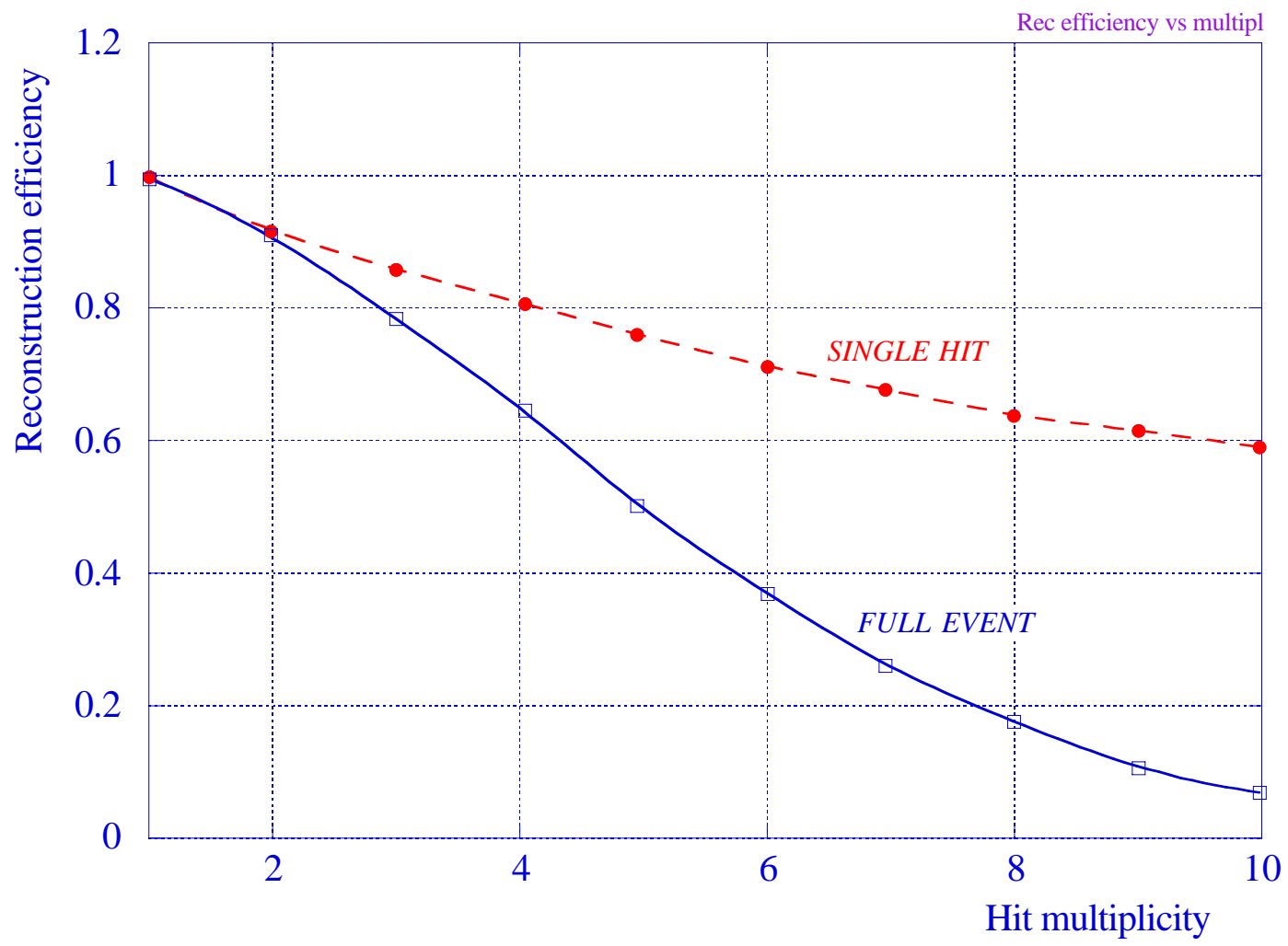

Fig. 51: Simulated multi-track reconstruction efficiency. 